\title{
A semi-analytical method for the dynamic analysis of cylindrical shells with arbitrary boundaries
}

\author{
Xu Liang ${ }^{1}$, Xing Zha ${ }^{1}$, Xue Jiang ${ }^{2, *}$, Zeng Cao ${ }^{1}$, Yuhong Wang ${ }^{1}$, Jianxing Leng ${ }^{1}$ \\ ${ }^{1}$ Ocean College, Zhejiang University, Hangzhou, Zhejiang 310058, China \\ ${ }^{2}$ Dept. of Naval Architecture, Ocean and Marine Engineering, University of Strathclyde, Henry \\ Dyer Building, Glasgow, G1 5AE, UK \\ * Corresponding Author: xue.jiang@ @strath.ac.uk
}

\begin{abstract}
The dynamic behavior of cylindrical shells with arbitrary boundaries is studied in this paper. Love's shell theory and Hamilton's principle are employed to derive the motion equations for cylindrical shells. A semi-analytical methodology, which incorporates Durbin's inverse Laplace transform, differential quadrature method and Fourier series expansion technique, is proposed to investigate this phenomenon. The use of the differential quadrature method provides a solution in terms of the axial direction whereas the use of Durbin's numerical inversion method generates a solution in the time domain. Comparison of calculated frequency parameters to that derived from the literature illustrates the effectiveness of the method. Specifically, convergence tests indicate that the present approach has a rapid convergence, the time-history response and the Navier's solution are in great agreement. Comparisons between time-history responses derived by two shell theories show that the results fit well with each other when the thickness-radius ratios are small enough. An analysis of the influences of boundaries on the time-history response of cylindrical shells indicates that the peak displacement is closely related to the degrees of freedom of boundaries. The influences of the length-radius ratios and the thickness-radius ratios on the peak displacement are further investigated.
\end{abstract}

Keywords: Time-history response; Frequency parameter; Differential quadrature method; Durbin's inverse Laplace transform.

\section{Introduction}

As an important structural component, the cylindrical shells have been widely used in ship construction and marine engineering, such as autonomous underwater vehicles, deep-ocean submersibles, subsea pipelines and nuclear facilities for their transportation ability, convenient storage and good load capacity (Gupta et al., 2016; Wu et al., 2018; Zhang et al., 2015a). These structures usually suffer from extreme loads including ocean currents, waves, and wind etc., resulting in vibration and damage of the structures (Zhang et al., 2015b). It is, therefore, necessary to exactly predict the vibration behavior of the cylindrical shells, as this knowledge can provide a theoretical foundation and scientific basis for structural design and performance optimization.

The extensive application of these structural elements has spurred rapid developments in shell theories and calculation methods for more accurate and efficient dynamic analysis of 
the cylindrical shells. The existing shell theories can be divided into three categories: the classical shell theory (CST), the first-order shear deformation theory (FSDT) and higher-order shear deformation theory (HSDT) (Thai and Kim, 2015). It is noted that CST is effective only in calculating the natural frequency and time-history response of thin cylindrical shells, FSDT and HSDT are more accurate in analyzing the dynamic behaviors of cylindrical shells. More details about the development of this research can be found in Leissa (1993), Qatu (2004) and Reddy (2003). Although the FSDT and HSDT are more accurate and precise, it is effective to choose CST to simulate the thin cylindrical shells under the consideration of computational loads and accuracy requirements.

Apart from the various shell theories, a variety of accurate and efficient calculation methods have been proposed one after another for vibration analysis of cylindrical shells, such as Rayleigh-Ritz method (Pradhan et al., 2000), Galerkin method (Haddadpour et al., 2007), discrete singular convolution method (Civalek, 2006, 2013), wave propagation approach (Liu et al., 2018), the transfer matrix method (Liang and Chen, 2006), finite element method (Kadoli and Ganesan, 2006; Santos et al., 2009), the generalized integral transform technique (Gu et al., 2013), meshless method (Ferreira et al., 2011; Ferreira et al., 2006), a domain decomposition approach ( $\mathrm{Qu}$ et al., 2013a; Qu et al., 2013b) and homotopy perturbation method (Yazdi, 2013) and so on. These methods mentioned above are proposed to derive the natural frequencies of shells and plates. However, the time-history response of cylindrical shells is rarely discussed in references. Reddy and Khdeir (1989) used the state space approach and the separation of variables technique to investigate the dynamic response of simply supported cross-ply laminated shallow shells under various loadings based on the third-order shear deformation shell theory. Lu and Lam (1995) combined the Rayleigh-Ritz method and the normal mode superposition method to evaluate the transient response of clamped laminated curved panels subjected to external loadings. Qing et al. (2008) presented the natural frequency, harmonic vibration and complex frequency response of simply supported laminated plates and shells with damping using the precise integration method and Muller method. Bodaghi and Shakeri (2012) developed an analytical approach to obtain the free vibration and dynamic response of the simply supported functionally graded piezo-electric cylindrical panel impacted by time-dependent blast pulses. Maleki et al. (2012) proposed a hybrid method to study the static and transient response of the moderately thick laminated cylindrical shell panels with various boundary conditions and loadings. Shao et al. (2017b) obtained the natural frequencies and transient response of cylindrical shells based on a simple first-order shear deformation shell theory using the method of reverberation ray matrix. Shao et al. (2017a) adopted an enhanced reverberation ray matrix approach to assess the transient response of composite laminated shallow shells with general boundary conditions based on the first-order shear deformation shallow shell theory and the classical shallow shell theory. Frikha et al. (2018) used a linear discrete double directors finite element model and Newmark's algorithm to study the dynamic behavior of functionally graded carbon nanotubes-reinforced shells. Duc (2013) incorporated the Bubnov-Galerkin method and Runge-Kutta method to evaluate the nonlinear transient response of imperfect shallow shells. Hajmohammad et al. (2017) chose the differential quadrature method and Newmark approach to investigate the dynamic response of submerged cylindrical shells under seismic action. Zhang et al. (2017) estimated the transient response of CVT-reinforced cylindrical shells 
subjected to impact loads by Fourier series expansion and Laplace transform.

Although the above methods can analyze the dynamic behavior of the cylindrical shells, it is difficult for them to take account of the calculation accuracy, efficiency and algorithm stability at the same time. Considering the limitations of these methods, a more precise and accurate method is needed to calculate the time-history response of cylindrical shells under arbitrary boundaries. In the present work, Love's shell theory and Hamilton's principle are employed to obtain the motion equations and a semi-analytical method that incorporates the differential quadrature method, Durbin's inverse Laplace transform is developed to predict the time-history response of cylindrical shells subjected to a harmonic load. This response is validated by comparing with the Navier's solution. The frequency parameters of cylindrical shells are then calculated and the influences of boundaries, length-radius ratios, and thickness-radius ratios on the time-history response of cylindrical shells are evaluated.

\section{Laplace transform and differential quadrature method}

\subsection{Laplace transform and its numerical inversion}

As an efficient and high precision computing method, the Laplace transform, which has been extensively used for solving partial differential equations, is expressed as follows:

$$
\tilde{h}(s)=\int_{0}^{+\infty} h(t) e^{-s t} d t
$$

in which $\sim$ denotes the transformed function and $s$ is a complex number.

Durbin developed a novel numerical inverse Laplace transform, which can give exact values of original functions in the interval $(0, T / 2)$, defined as (Durbin, 1974):

$$
h(t) \approx \frac{2 e^{\lambda t}}{T}\left\{\tilde{h}(\lambda) / 2+\sum_{k=1}^{K} \operatorname{Re}[\tilde{h}(\lambda+k \pi i / T)] \cos (k \pi t / T)\right\}
$$

in which $\lambda=5 / T, i$ is the imaginary unit in complex number and $K$ denotes a large integer.

\subsection{Differential quadrature method}

The principle of differential quadrature method is that the weighted sum of functional values of all grid points is used to represent a function and its derivatives at a chosen point, and it is defined as below (Liang et al., 2014):

$$
\frac{\partial^{i} g\left(x_{m}\right)}{\partial x^{i}}=\sum_{n=1}^{N} C_{m n}^{(i)} g\left(x_{n}\right) \quad(m, n=1,2, . . N)
$$

in which $N$ denotes the number of grid points, and $C_{\mathrm{mn}}^{(i)}$ refer to the weighting coefficients defined by:

$$
\begin{gathered}
C_{m n}^{(1)}=\frac{\prod_{r=1, r \neq n}^{N}\left(x_{m}-x_{r}\right)}{\left(x_{m}-x_{n}\right) \prod_{r=1, r \neq n}^{N}\left(x_{n}-x_{r}\right)} \\
C_{m n}^{(i)}=i\left(C_{m n}^{(i-1)} C_{m n}^{1}-\frac{C_{m n}^{(i-1)}}{x_{m}-x_{n}}\right)(i=2,3, \ldots N-1)
\end{gathered}
$$

in which $n \neq m$, and the $C_{\mathrm{mm}}^{(\mathrm{i})}$ are given as: 


$$
C_{m m}^{(i)}=-\sum_{n=1, n \neq m}^{N} C_{m n}^{(i)}(m=1,2 \ldots N, i=1,2 \ldots N-1)
$$

\section{Problem description}

\subsection{Governing equations}

A thin cylindrical shell as shown in Fig. 1 is considered, the symbols $l, R, h$ denote the length, radius, and thickness of the cylindrical shell. The origin of the cylindrical coordinate system is fixed at the mid-surface of the cylindrical shells.

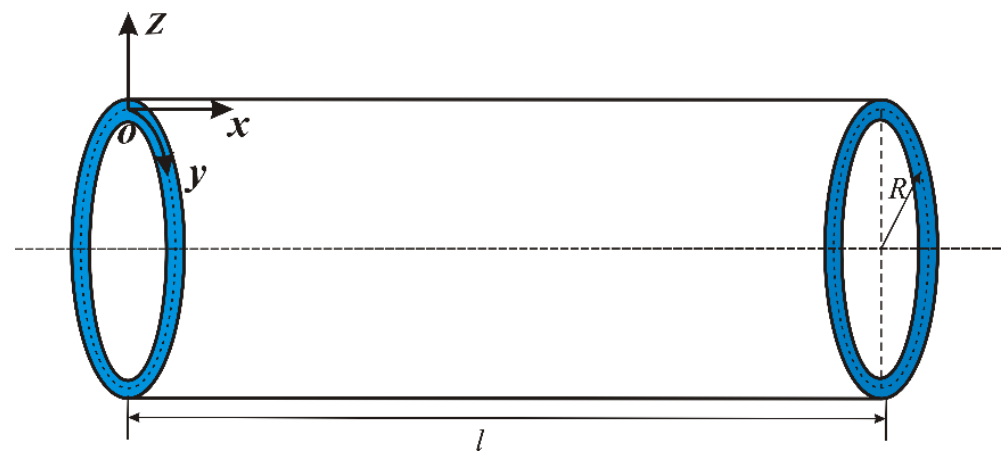

Fig. 1. Geometry of the thin cylindrical shell subjected to a harmonic load.

For thin shells, the following assumptions are considered: the shear deformation and rotary inertia are ignored, and the normal line of the mid-surface remains straight and normals to the mid-surface during deformation. The displacement-strain relationships based on Love's shell theory are expressed as:

$$
\boldsymbol{\varepsilon}=\left\{\begin{array}{c}
\varepsilon_{x} \\
\varepsilon_{\theta} \\
\gamma_{x \theta}
\end{array}\right\}=\boldsymbol{\varepsilon}_{0}+z \mathbf{\kappa}=\left\{\begin{array}{c}
\frac{\partial u_{0}}{\partial x} \\
\frac{1}{R}\left(\frac{\partial v_{0}}{\partial \theta}+w_{0}\right) \\
\frac{\partial v_{0}}{\partial x}+\frac{1}{R} \frac{\partial u_{0}}{\partial \theta}
\end{array}\right\}+z\left\{\begin{array}{c}
-\frac{\partial^{2} w_{0}}{\partial x^{2}} \\
\frac{1}{R^{2}}\left(\frac{\partial v_{0}}{\partial \theta}-\frac{\partial^{2} w_{0}}{\partial \theta^{2}}\right) \\
\frac{2}{R}\left(\frac{\partial v_{0}}{\partial x}-\frac{\partial^{2} w_{0}}{\partial x \partial \theta}\right)
\end{array}\right\}
$$

in which $\boldsymbol{\varepsilon}_{\mathbf{0}}$ is the strain vector and $\boldsymbol{\kappa}$ refers to the vector of curvature changes, $u_{0}, v_{0}, w_{0}$ denote displacement components on the mid-surface in $x$-, $\theta$ - and $z$-directions, respectively;

The stress-strain relationships are given as:

$$
\boldsymbol{\sigma}=\mathbf{Q} \varepsilon
$$

in which $\boldsymbol{\sigma}$ is the stress vector, and $\mathbf{Q}$ is the elastic constants matrix defined as:

$$
\boldsymbol{\sigma}=\left\{\sigma_{x}, \sigma_{\theta}, \tau_{x \theta}\right\}, \mathbf{Q}=\left[\begin{array}{ccc}
Q_{11} & Q_{12} & 0 \\
Q_{12} & Q_{22} & 0 \\
0 & 0 & Q_{66}
\end{array}\right]
$$

For isotropic materials, the elastic constants $Q_{i j}(i, j=1,2$ and 6$)$ can be stated as:

$$
Q_{11}=Q_{22}=\frac{E}{1-\mu^{2}}, Q_{12}=\frac{\mu E}{1-\mu^{2}}, Q_{66}=\frac{E}{2(1+\mu)} .
$$

in which $E$ is Young's modulus, and $\mu$ is Poisson's ratio. 
The Hamilton's principle is employed to obtain motion equations for thin cylindrical shells, and it is defined as:

$$
\delta J=\int_{0}^{t}\left(\delta K-\delta V+\delta W_{n c}\right) d t=0
$$

in which $K$, and $V$ refer to the kinetic energy and strain energy, $W_{n c}$ denotes the work done by external loads.

The kinetic energy without considering the rotary inertia terms can be written as follows:

$$
K=\frac{1}{2} \int_{0}^{l} \int_{0}^{2 \pi} \int_{-h / 2}^{h / 2} \rho\left(\dot{u}_{0}^{2}+\dot{v}_{0}^{2}+\dot{w}_{0}^{2}\right) R d x d \theta d z
$$

in which the superscript " " " represents the variables with respect to time.

The strain energy is expressed as:

$$
V=\frac{1}{2} \int_{0}^{l} \int_{0}^{2 \pi} \int_{-h / 2}^{h / 2}\left(\sigma_{x} \varepsilon_{x}+\sigma_{\theta} \varepsilon_{\theta}+\tau_{x \theta} \gamma_{x \theta}\right) R d x d \theta d z
$$

The work done by external loads is defined by:

$$
\delta W_{n c}=\int_{0}^{l} \int_{0}^{2 \pi}\left(f_{x} \delta u_{0}+f_{\theta} \delta v_{0}+f_{z} \delta w_{0}\right) R d x d \theta
$$

in which $f_{x}, f_{\theta}$ and $f_{z}$ stand for external loads in $x$-, $\theta$ - and $z$-directions, respectively, and it is noteworthy that $f_{x}=0, f_{\theta}=0, f_{z}=3000 \cos (300 \mathrm{t}) \delta(\theta)$.

Substituting Eqs. (12)-(14) into Eq. (11), the motion equations for thin cylindrical shells in terms of stress resultants are given as:

$$
\begin{gathered}
\frac{\partial N_{x}}{\partial x}+\frac{1}{R} \frac{\partial N_{x \theta}}{\partial \theta}+f_{x}=I_{0} \frac{\partial^{2} u_{0}}{\partial t^{2}} \\
\frac{\partial N_{x \theta}}{\partial x}+\frac{1}{R} \frac{\partial N_{\theta}}{\partial \theta}+\frac{1}{R} \frac{\partial M_{x \theta}}{\partial x}+\frac{1}{R^{2}} \frac{\partial M_{\theta}}{\partial \theta}+f_{\theta}=I_{0} \frac{\partial^{2} v_{0}}{\partial t^{2}} \\
\frac{\partial^{2} M_{x}}{\partial x^{2}}+\frac{2}{R} \frac{\partial^{2} M_{x \theta}}{\partial x \partial \theta}+\frac{1}{R^{2}} \frac{\partial^{2} M_{\theta}}{\partial \theta^{2}}-\frac{N_{\theta}}{R}+f_{z}=I_{0} \frac{\partial^{2} w_{0}}{\partial t^{2}}
\end{gathered}
$$

in which

$$
\begin{aligned}
\left\{N_{x}, N_{\theta}, N_{x \theta}\right\} & =\int_{-h / 2}^{h / 2}\left\{\sigma_{x}, \sigma_{\theta}, \tau_{x \theta}\right\} d z \\
\left\{M_{x}, M_{\theta}, M_{x \theta}\right\} & =\int_{-h / 2}^{h / 2} z\left\{\sigma_{x}, \sigma_{\theta}, \tau_{x \theta}\right\} d z \\
I_{0} & =\int_{-h / 2}^{h / 2} \rho d z
\end{aligned}
$$

By substituting Eqs. (7)-(10) into Eq. (15), the motion equations in terms of the displacement components $\left(u_{0}, v_{0}, w_{0}\right)$ can be written as: 
in which

$$
\left\{A_{i j}, B_{i j}, D_{i j}\right\}=\int_{-h / 2}^{h / 2}\left\{1, z, z^{2}\right\} Q_{i j} d z, \quad(i, j=1,2,6) .
$$

\subsection{The boundary conditions at the edges}

The boundaries of the elastically restrained shell are described by setting the values of translational springs and rotational spring, and the boundaries of cylindrical shells can be stated as follows (Jin et al., 2015; Jin et al., 2013):

At left edge $(x=0)$ :

$$
\begin{gathered}
k_{u 0} u_{0}=N_{x}, \quad k_{v 0} v_{0}=N_{x \theta}+\frac{M_{x \theta}}{R}, \\
k_{w 0} w_{0}=Q_{x}+\frac{\partial M_{x \theta}}{R \partial \theta}, K_{w 0} \frac{\partial w_{0}}{\partial x}=-M_{x} .
\end{gathered}
$$

At right edge $(x=l)$ :

$$
\begin{gathered}
k_{u L} u_{0}=-N_{x}, \quad k_{v L} v_{0}=-N_{x \theta}-\frac{M_{x \theta}}{R}, \\
k_{w L} w_{0}=-Q_{x}-\frac{\partial M_{x \theta}}{R \partial \theta}, \quad K_{w L} \frac{\partial w_{0}}{\partial x}=M_{x} .
\end{gathered}
$$

in which $k_{u}, k_{v}$ and $k_{w}$ denote the translational spring stiffness coefficients, $K_{w}$ is the rotational spring stiffness.

The stiffness coefficients of springs corresponding to four boundaries are defined as:

Free edge $(\mathrm{F})$ :

$$
k_{u}=k_{v}=k_{w}=K_{w}=0
$$

Simply-supported edge (S):

$$
k_{u}=k_{v}=k_{w}=10^{14}, K_{w}=0 .
$$


Shear-diaphragm edge (SD):

$$
k_{u}=K_{w}=0, k_{v}=k_{w}=10^{14} .
$$

Clamped edge (C):

$$
k_{u}=k_{v}=k_{w}=K_{w}=10^{14} .
$$

\section{Solution process}

\subsection{Natural frequency solution}

The modal frequencies of cylindrical shells are calculated, and the displacement components of cylindrical shells for modal analysis are expanded as:

$$
\left\{\begin{array}{c}
u_{0}(x, \theta, t)=U(x) \cos (k \theta) e^{i \omega t}, \\
v_{0}(x, \theta, t)=V(x) \sin (k \theta) e^{i \omega t}, \\
w_{0}(x, \theta, t)=W(x) \cos (k \theta) e^{i \omega t} .
\end{array}\right.
$$

in which $k$ and $\omega$ denote the circumferential wavenumber and natural frequency of the cylindrical shell, respectively.

By substituting Eq. (27) into the motion equations without considering external forces, and then employing the DQM on the resulting equations, the following formulas can be obtained:

$$
\begin{gathered}
-\frac{A_{66} k^{2}}{R} U_{i}+A_{11} R \sum_{j=1}^{N} A_{i j}^{(2)} U_{j}+\left(A_{12} k+A_{66} k+\frac{B_{12} k}{R}+\frac{2 B_{66} k}{R}\right) \sum_{j=1}^{N} A_{i j}^{(1)} V_{j} \\
+\left(A_{12}+\frac{B_{12} k^{2}}{R}+\frac{2 B_{66} k^{2}}{R}\right) \sum_{j=1}^{N} A_{i j}^{(1)} W_{j}-B_{11} R \sum_{j=1}^{N} A_{i j}^{(3)} W_{j}+\omega^{2} I_{0} R U_{i}=0, \\
\left(-A_{12} k-A_{66} k-\frac{B_{12} k}{R}-\frac{2 B_{66} k}{R}\right) \sum_{j=1}^{N} A_{i j}^{(1)} U_{j}+\left(-\frac{D_{22} k^{2}}{R^{3}}-\frac{2 B_{22} k^{2}}{R^{2}}-\frac{A_{22} k^{2}}{R}\right) V_{i} \\
+\left(4 B_{66}+\frac{4 D_{66}}{R}+A_{66} R\right) \sum_{j=1}^{N} A_{i j}^{(2)} V_{j}+\left(-\frac{D_{22} k^{3}}{R^{3}}-\frac{B_{22} k}{R^{2}}-\frac{B_{22} k^{3}}{R^{2}}-\frac{A_{22} k}{R}\right) W_{i} \\
+\left(B_{12} k+2 B_{66} k+\frac{D_{12} k}{R}+\frac{4 D_{66} k}{R}\right) \sum_{j=1}^{N} A_{i j}^{(2)} W_{j}+\omega^{2} I_{0} R V_{i}=0, \\
\left.+-A_{12}-\frac{B_{12} k^{2}}{R}-\frac{2 B_{66} k^{2}}{R}\right) \sum_{j=1}^{N} A_{i j}^{(1)} U_{j}+B_{11} R \sum_{j=1}^{N} A_{i j}^{(3)} U_{j}+\left(-\frac{D_{22} k^{3}}{R^{3}}-\frac{B_{22} k}{R^{2}}-\frac{B_{22} k^{3}}{R^{2}}-\frac{A_{22} k}{R}\right) V_{i} \\
+\left(B_{12} k+2 B_{66} k+\frac{D_{12} k}{R}+\frac{4 D_{66} k}{R}\right) \sum_{j=1}^{N} A_{i j}^{(2)} V_{j}+\left(-\frac{D_{22} k^{4}}{R^{3}}-\frac{2 B_{22} k^{2}}{R^{2}}-\frac{A_{22}}{R}\right) W_{i} \\
+\left(2 B_{12}+\frac{2 D_{12} k^{2}}{R}+\frac{4 D_{66} k^{2}}{R}\right) \sum_{j=1}^{N} A_{i j}^{(2)} W_{j}-D_{11} R \sum_{j=1}^{N} A_{i j}^{(4)} W_{j}+\omega^{2} I_{0} R W_{i}=0 .
\end{gathered}
$$

Substituting boundaries into Eq. (28), the obtained equations are rewritten into matrix form as: 


$$
\begin{gathered}
{\left[\begin{array}{ll}
\mathbf{k}_{\mathbf{b b}} & \mathbf{k}_{\mathbf{b d}} \\
\mathbf{k}_{\mathbf{d b}} & \mathbf{k}_{\mathbf{d d}}
\end{array}\right]\left\{\begin{array}{l}
\mathbf{u}_{\mathbf{b}} \\
\mathbf{u}_{\mathbf{d}}
\end{array}\right\}+\omega^{2}\left[\begin{array}{ll}
\mathbf{s}_{\mathbf{b b}} & \mathbf{s}_{\mathbf{b d}} \\
\mathbf{s}_{\mathbf{d b}} & \mathbf{s}_{\mathbf{d d}}
\end{array}\right]\left\{\begin{array}{l}
\mathbf{u}_{\mathbf{b}} \\
\mathbf{u}_{\mathbf{d}}
\end{array}\right\}=\left\{\begin{array}{l}
\mathbf{0} \\
\mathbf{0}
\end{array}\right\}} \\
\mathbf{u}_{\mathbf{b}}^{\mathbf{T}}=\left\{U_{1}, U_{n}, V_{1}, V_{n}, W_{1}, W_{2}, W_{n-1}, W_{n}\right\}, \\
\mathbf{u}_{\mathbf{d}}^{\mathbf{T}}=\left\{U_{2}, \ldots, U_{n-1}, V_{2}, \ldots, V_{n-1}, W_{3}, \ldots, W_{n-2}\right\} .
\end{gathered}
$$

After eliminating the vector $\boldsymbol{u}_{\boldsymbol{b}}$, the resulting equations can be described as:

$$
\left(\omega^{2} \mathbf{M}+\mathbf{K}\right) \mathbf{u}_{\mathbf{d}}=\mathbf{0}
$$

in which $\boldsymbol{M}$ denotes the mass matrix, $\boldsymbol{K}$ is stiffness matrix, and $\boldsymbol{u}_{\boldsymbol{d}}$ refer to a vector. The eigenvalues of the following matrix are the natural frequencies, and the eigenvalue matrix is given as:

$$
\mathbf{E}=\left[\begin{array}{cc}
\mathbf{0} & \mathbf{I} \\
-\mathbf{M}^{-1} \mathbf{K} & \mathbf{0}
\end{array}\right]
$$

\subsection{Transient response solution}

The trigonometric series are taken to expand the displacement components and external loads on the mid-surface of the cylindrical shells in the circumferential direction, and the detailed expansion forms of displacement components and external loads are presented as:

$$
\begin{array}{ll}
\tilde{u}_{0}=\sum_{k=1}^{\infty} \tilde{\overline{\bar{u}}}_{0}(x, s) \cos k \theta, & \tilde{f}_{x}=\sum_{k=1}^{\infty} \tilde{\overline{\bar{f}}}(x, s) \cos k \theta, \\
\tilde{v}_{0}=\sum_{k=1}^{\infty} \tilde{\bar{v}}_{0}(x, s) \sin k \theta, & \tilde{f}_{\theta}=\sum_{k=1}^{\infty} \tilde{\bar{f}}_{\theta}(x, s) \sin k \theta, \\
\tilde{w}_{0}=\sum_{k=1}^{\infty} \tilde{\bar{w}}_{0}(x, s) \cos k \theta, & \tilde{f}_{z}=\sum_{k=1}^{\infty} \tilde{\bar{f}}_{z}(x, s) \cos k \theta .
\end{array}
$$

Substituting Eq. (32) into Eq. (19) and the motion equations processed by Laplace transform are expressed as: 


$$
\begin{gathered}
\left(-\frac{A_{66} k^{2}}{R}-I_{0} R s^{2}\right) \tilde{\bar{u}}_{0}+A_{11} R \frac{\partial^{2} \tilde{\bar{u}}_{0}}{\partial x^{2}}+\left(A_{12} k+A_{66} k+\frac{B_{12} k}{R}+\frac{2 B_{66} k}{R}\right) \frac{\partial \tilde{\bar{v}}_{0}}{\partial x} \\
+\left(A_{12}+\frac{B_{12} k^{2}}{R}+\frac{2 B_{66} k^{2}}{R}\right) \frac{\partial \tilde{\bar{w}}_{0}}{\partial x}-B_{11} R \frac{\partial^{3} \tilde{\bar{w}}_{0}}{\partial x^{3}}+\tilde{\bar{f}}_{x} R=0, \\
\left(-A_{12} k-A_{66} k-\frac{B_{12} k}{R}-\frac{2 B_{66} k}{R}\right) \frac{\partial \tilde{\bar{u}}_{0}}{\partial x}+\left(4 B_{66}+\frac{4 D_{66}}{R}+A_{66} R\right) \frac{\partial^{2} \tilde{\bar{v}}_{0}}{\partial x^{2}} \\
+\left(-\frac{D_{22} k^{2}}{R^{3}}-\frac{2 B_{22} k^{2}}{R^{2}}-\frac{A_{22} k^{2}}{R}-I_{0} R s^{2}\right) \tilde{\bar{v}}_{0}+\left(-\frac{D_{22} k^{3}}{R^{3}}-\frac{B_{22} k}{R^{2}}-\frac{B_{22} k^{3}}{R^{2}}-\frac{A_{22} k}{R}\right) \tilde{\bar{w}}_{0} \\
+\left(B_{12} k+2 B_{66} k+\frac{D_{12} k}{R}+\frac{4 D_{66} k}{R}\right) \frac{\partial^{2} \tilde{\bar{w}}_{0}}{\partial x^{2}}+\tilde{\bar{f}}_{\theta} R=0, \\
\left(-A_{12}-\frac{B_{12} k^{2}}{R}-\frac{2 B_{66} k^{2}}{R}\right) \frac{\partial \tilde{\bar{u}}_{0}}{\partial x}+B_{11} R \frac{\partial^{3} \tilde{\bar{u}}_{0}}{\partial x^{3}}+\left(-\frac{D_{22} k^{3}}{R^{3}}-\frac{B_{22} k}{R^{2}}-\frac{B_{22} k^{3}}{R^{2}}-\frac{A_{22} k}{R}\right) \tilde{\bar{v}}_{0}+ \\
\left(B_{12} k+2 B_{66} k+\frac{D_{12} k}{R}+\frac{4 D_{66} k}{R}\right) \frac{\partial^{2} \tilde{\bar{v}}_{0}}{\partial x^{2}}+\left(-\frac{D_{22} k^{4}}{R^{3}}-\frac{2 B_{22} k^{2}}{R^{2}}-\frac{A_{22}}{R}-I_{0} R s^{2}\right) \tilde{\bar{w}}_{0} \\
+\left(2 B_{12}+\frac{2 D_{12} k^{2}}{R}+\frac{4 D_{66} k^{2}}{R}\right) \frac{\partial^{2} \tilde{\bar{w}}_{0}}{\partial x^{2}}-D_{11} R \frac{\partial^{4} \tilde{\bar{w}}_{0}}{\partial x^{4}}+\tilde{\bar{f}}_{z} R=0 .
\end{gathered}
$$

Employing the DQM on Eq. (33) and the following equations can be given:

$$
\begin{gathered}
\left(-\frac{A_{66} k^{2}}{R}-I_{0} R s^{2}\right) \tilde{\bar{u}}_{0 i}+A_{11} R \sum_{j=1}^{N} A_{i j}^{(2)} \tilde{\bar{u}}_{0 j}+\left(A_{12} k+A_{66} k+\frac{B_{12} k}{R}+\frac{2 B_{66} k}{R}\right) \sum_{j=1}^{N} A_{i j}^{(1)} \tilde{\bar{v}}_{0 j} \\
+\left(A_{12}+\frac{B_{12} k^{2}}{R}+\frac{2 B_{66} k^{2}}{R}\right) \sum_{j=1}^{N} A_{i j}^{(1)} \tilde{\bar{w}}_{0 j}-B_{11} R \sum_{j=1}^{N} A_{i j}^{(3)} \tilde{\bar{w}}_{0 j}+\tilde{\bar{f}}_{x i} R=0, \\
\left(-A_{12} k-A_{66} k-\frac{B_{12} k}{R}-\frac{2 B_{66} k}{R}\right) \sum_{j=1}^{N} A_{i j}^{(1)} \tilde{\bar{u}}_{0 j}+\left(4 B_{66}+\frac{4 D_{66}}{R}+A_{66} R\right) \sum_{j=1}^{N} A_{i j}^{(2)} \tilde{\bar{v}}_{0 j} \\
+\left(-\frac{D_{22} k^{2}}{R^{3}}-\frac{2 B_{22} k^{2}}{R^{2}}-\frac{A_{22} k^{2}}{R}-I_{0} R s^{2}\right) \tilde{\bar{v}}_{0 i}+\left(-\frac{D_{22} k^{3}}{R^{3}}-\frac{B_{22} k}{R^{2}}-\frac{B_{22} k^{3}}{R^{2}}-\frac{A_{22} k}{R}\right) \tilde{\bar{w}}_{0 i} \\
+\left(B_{12} k+2 B_{66} k+\frac{D_{12} k}{R}+\frac{4 D_{66} k}{R}\right) \sum_{j=1}^{N} A_{i j}^{(2)} \tilde{\bar{w}}_{0 j}+\tilde{\bar{f}}_{\theta i} R=0, \\
\left(-A_{12}-\frac{B_{12} k^{2}}{R}-\frac{2 B_{66} k^{2}}{R}\right) \sum_{j=1}^{N} A_{i j}^{(1)} \tilde{\bar{u}}_{0 j}+B_{11} R \sum_{j=1}^{N} A_{i j}^{(3)} \tilde{\bar{u}}_{0 j}+\left(-\frac{D_{22} k^{3}}{R^{3}}-\frac{B_{22} k}{R^{2}}-\frac{B_{22} k^{3}}{R^{2}}-\frac{A_{22} k}{R}\right) \tilde{\bar{v}}_{0 i}+ \\
\left(B_{12} k+2 B_{66} k+\frac{D_{12} k}{R}+\frac{4 D_{66} k}{R}\right) \sum_{j=1}^{N} A_{i j}^{(2)} \tilde{\bar{v}}_{0 j}+\left(-\frac{D_{22} k^{4}}{R^{3}}-\frac{2 B_{22} k^{2}}{R^{2}}-\frac{A_{22}}{R}-I_{0} R s^{2}\right) \tilde{\bar{w}}_{0 i} \\
+\left(2 B_{12}+\frac{2 D_{12} k^{2}}{R}+\frac{4 D_{66} k^{2}}{R}\right) \sum_{j=1}^{N} A_{i j}^{(2)} \tilde{\bar{w}}_{0 j}-D_{11} R \sum_{j=1}^{N} A_{i j}^{(4)} \tilde{\bar{w}}_{0 j}+\tilde{\bar{f}}_{z i} R=0 .
\end{gathered}
$$

The matrix form of Eq. (34) can be stated as:

$$
\mathbf{H} \cdot \mathbf{U}=\mathbf{F}
$$

in which 


$$
\begin{gathered}
\mathbf{U}=\left\{\begin{array}{c}
\mathbf{u}_{\mathbf{0}} \\
\mathbf{v}_{\mathbf{0}} \\
\mathbf{w}_{\mathbf{0}}
\end{array}\right\}, \mathbf{F}=\left\{\begin{array}{c}
\mathbf{f}_{\mathbf{x}} \\
\mathbf{f}_{\boldsymbol{\theta}} \\
\mathbf{f}_{\mathbf{z}}
\end{array}\right\}, \mathbf{H}=\left\{\begin{array}{lll}
\mathbf{H}_{\mathbf{1 1}} & \mathbf{H}_{\mathbf{1 2}} & \mathbf{H}_{\mathbf{1 3}} \\
\mathbf{H}_{\mathbf{2 1}} & \mathbf{H}_{\mathbf{2 2}} & \mathbf{H}_{\mathbf{2 3}} \\
\mathbf{H}_{\mathbf{3 1}} & \mathbf{H}_{\mathbf{3 2}} & \mathbf{H}_{\mathbf{3 3}}
\end{array}\right\}, \\
\mathbf{u}_{\mathbf{0}}^{T}=\left\{\tilde{\overline{\bar{u}}}_{01}, \ldots \tilde{\overline{\bar{u}}}_{0 j}, \ldots \tilde{\overline{\bar{u}}}_{0 N}\right\}, \mathbf{v}_{\mathbf{0}}^{T}=\left\{\tilde{\overline{\bar{v}}}_{01}, \ldots \tilde{\overline{\bar{v}}}_{0 j}, \ldots \tilde{\overline{\bar{v}}}_{0 N}\right\}, \mathbf{w}_{\mathbf{0}}^{T}=\left\{\tilde{\overline{\bar{w}}}_{01}, \ldots \tilde{\overline{\bar{w}}}_{0 j}, \ldots \tilde{\overline{\bar{w}}}_{0 N}\right\}, \\
\mathbf{f}_{\mathbf{x}}^{T}=-R\left\{\tilde{\overline{\bar{f}}}_{x 1}, \ldots \tilde{\bar{f}}_{x j}, \ldots \tilde{\bar{f}}_{x N}\right\}, \mathbf{f}_{\boldsymbol{\theta}}^{T}=-R\left\{\tilde{\overline{\bar{f}}}_{\theta 1}, \ldots \tilde{\bar{f}}_{\theta j}, \ldots \tilde{\bar{f}}_{\theta N}\right\}, \mathbf{f}_{\mathbf{z}}^{T}=-R\left\{\tilde{\bar{f}}_{z 1}, \ldots \tilde{\bar{f}}_{z j}, \ldots \tilde{\bar{f}}_{z N}\right\} .
\end{gathered}
$$

The expressions of matrix $\mathbf{H}$ are detailed in Appendix A.

The general boundaries of the elastically restrained shells processed by the trigonometric series expansion and Laplace transform can be stated as $(x=0)$ :

$$
\begin{gathered}
T_{11} \frac{\partial \tilde{\bar{u}}_{0}}{\partial x}+T_{12} \tilde{\bar{v}}_{0}+T_{13} \tilde{\bar{w}}_{0}=k_{u 0} \tilde{\bar{u}}_{0}, \\
T_{21} \tilde{\bar{u}}_{0}+T_{22} \frac{\partial \tilde{\bar{v}}_{0}}{\partial x}+T_{23} \frac{\partial \tilde{\bar{w}}_{0}}{\partial x}=k_{v 0} \tilde{\bar{v}}_{0}, \\
T_{32} \frac{\partial \tilde{\bar{v}}_{0}}{\partial x}+T_{331} \frac{\partial \tilde{\bar{w}}_{0}}{\partial x}+T_{332} \frac{\partial^{3} \tilde{\bar{w}}_{0}}{\partial x^{3}}=k_{w 0} \tilde{\bar{w}}_{0}, \\
T_{42} \tilde{\bar{v}}_{0}+T_{431} \tilde{\overline{\bar{w}}}_{0}+T_{432} \frac{\partial^{2} \tilde{\bar{w}}_{0}}{\partial x^{2}}=K_{w 0} \frac{\partial \tilde{\bar{w}}_{0}}{\partial x} .
\end{gathered}
$$

in which

$$
\begin{gathered}
T_{11}=\frac{E h}{1-\mu^{2}}, T_{12}=\frac{E h}{1-\mu^{2}} \frac{\mu k}{R}, T_{13}=\frac{E h}{1-\mu^{2}} \frac{\mu}{R}, T_{21}=-\frac{k}{R} \frac{E h}{2(1+\mu)}, \\
T_{22}=\frac{E h}{2(1+\mu)}\left(1+\frac{h^{2}}{6 R^{2}}\right), T_{23}=\frac{E h}{2(1+\mu)} \frac{h^{2} k}{6 R^{2}}, T_{32}=\frac{E h^{3} k}{12\left(1-\mu^{2}\right)}\left(\frac{\mu}{2 R^{2}}+\frac{1-\mu}{R^{2}}\right), \\
T_{331}=\frac{E h^{3} k^{2}}{12\left(1-\mu^{2}\right)}\left(\frac{\mu}{2 R^{2}}+\frac{1-\mu}{R^{2}}\right), T_{332}=-\frac{1}{2} \frac{E h^{3}}{12\left(1-\mu^{2}\right)}, \\
T_{42}=-\frac{\mu k}{R^{2}} \frac{E h^{3}}{12\left(1-\mu^{2}\right)}, T_{431}=-\frac{\mu k^{2}}{R^{2}} \frac{E h^{3}}{12\left(1-\mu^{2}\right)}, T_{432}=\frac{E h^{3}}{12\left(1-\mu^{2}\right)} .
\end{gathered}
$$

By substituting Eq. (37) into Eq. (35), and the elements corresponding to boundaries in matrices $\mathbf{H}_{\mathrm{ij}}$ are available in Appendix B.

After finding the solution of Eq. (35), the time-history responses of cylindrical shells are obtained by using the Durbin's inverse Laplace transform.

\section{Numerical results}

A Mathematica package was developed to present the numerical results in this paper, and the following steps are taken to evaluate the model. Firstly, the frequency parameters of thin cylindrical shells are calculated. Subsequently, the convergence of results for different numbers of grid points $(N)$ in the axial direction and the validity of the present method is estimated. Thirdly, the time-history responses derived by classical shell theory and first-order shear deformation theory are compared. Fourthly, the influences of various boundaries on cylindrical shell displacement are investigated. Lastly, the influences of length-radius ratios $(l / R)$ and thickness-radius ratios $(h / R)$ on time-history response are assessed. Considering a set 
of model parameters used in following numerical calculation, the system parameters used in the present work are given as follows: $E=70 \mathrm{GPa}, \mu=0.3, \rho=2707 \mathrm{~kg} / \mathrm{m}^{3}, l=2 \mathrm{~m}, R=0.5 \mathrm{~m}, h=0.002 \mathrm{~m}$.

In order to guarantee the convergence and accuracy of the results, the Chebyshev-Gauss-Lobatto grid points are used in present work and are defined as:

$$
x_{\kappa}=\frac{l}{2}\left(1-\cos \left(\frac{\kappa-1}{N-1}\right) \pi\right), \kappa=1,2, \ldots N
$$

in which $N$ denotes the number of grid points in the axial direction, and it is taken as 11 in the following numerical examples.

\subsection{Natural frequencies}

To prove the correctness of the present method for the modal analysis of thin cylindrical shells, a series of calculated results will be compared with the frequency parameters from the literature. The length-radius ratio $l / R=20$ and Poisson's ratio $\mu=0.3$ are adopted in the following calculations. Table 1 presents the frequency parameters of cylindrical shells under three different boundaries, $m$ and $k$ refer to the axial and circumferential wavenumbers, respectively. It can be found from Table 1 that the frequency parameters produced by the differential quadrature method are consistent with the results obtained by Loy et al. (1997). Table 2 shows the frequency parameters of a SD-SD cylindrical shell under different thickness-radius ratios. As shown in Table 2, the frequency parameters generated by the proposed method fits well with the results calculated by Markuš (1988) and Loy et al. (1997). The frequency parameters of a different cylindrical shell with C-SD boundary are again compared in Table 3, which also shows a good agreement between the results produced by the present method and results from the literature.

Table 1 Frequency parameters of a thin cylindrical shell with three different boundaries $(h / R=0.01)$.

\begin{tabular}{ccccccc}
\hline & \multicolumn{5}{c}{ Frequency parameters $\Omega=\omega R \sqrt{\left(1-\mu^{2}\right) \rho / E}$} \\
\cline { 2 - 7 }$k$ & \multicolumn{2}{c}{ SD-SD } & \multicolumn{2}{c}{ C-C } & \multicolumn{2}{c}{ C-SD } \\
\cline { 2 - 7 } & $\begin{array}{c}\text { Loy et al. } \\
(1997)\end{array}$ & Present & $\begin{array}{c}\text { Loy et al. } \\
(1997)\end{array}$ & Present & $\begin{array}{c}\text { Loy et al. } \\
(1997)\end{array}$ & Present \\
\hline 1 & 0.016101 & 0.016101 & 0.032885 & 0.032884 & 0.023974 & 0.023976 \\
2 & 0.009382 & 0.009378 & 0.013923 & 0.013929 & 0.011225 & 0.011221 \\
3 & 0.022105 & 0.022103 & 0.022672 & 0.022668 & 0.022310 & 0.022308 \\
4 & 0.042095 & 0.042094 & 0.042208 & 0.042207 & 0.042139 & 0.042137 \\
5 & 0.068008 & 0.068007 & 0.068046 & 0.068045 & 0.068024 & 0.068023 \\
6 & 0.099730 & 0.099729 & 0.099748 & 0.099747 & 0.099738 & 0.099737 \\
7 & 0.137239 & 0.137238 & 0.137249 & 0.137249 & 0.137244 & 0.137243 \\
8 & 0.180527 & 0.180527 & 0.180535 & 0.180534 & 0.180531 & 0.180531 \\
9 & 0.229594 & 0.229593 & 0.229599 & 0.229598 & 0.229596 & 0.229596 \\
10 & 0.284435 & 0.284435 & 0.284439 & 0.284439 & 0.284437 & 0.284437 \\
\hline
\end{tabular}

Table 2 Frequency parameters of SD-SD cylindrical shells. 
Frequency parameters $\Omega=\omega R \sqrt{\left(1-\mu^{2}\right) \rho / E}$

\begin{tabular}{ccccccc}
\hline & \multicolumn{3}{c}{$h / R=0.05$} & & \multicolumn{3}{c}{$h / R=0.002$} \\
\cline { 2 - 7 }$k$ & $\begin{array}{c}\text { Markuš } \\
(1988)\end{array}$ & $\begin{array}{c}\text { Loy et al. } \\
(1997)\end{array}$ & Present & $\begin{array}{c}\text { Markuš } \\
(1988)\end{array}$ & $\begin{array}{c}\text { Loy et al. } \\
(1997)\end{array}$ & Present \\
\hline 1 & 0.0161063 & 0.01610 & 0.0161029 & 0.0161011 & 0.016101 & 0.0161011 \\
2 & 0.0392332 & 0.03930 & 0.0392710 & 0.00545243 & 0.005453 & 0.00545297 \\
3 & 0.109477 & 0.109824 & 0.1098116 & 0.00503724 & 0.005042 & 0.00504148 \\
4 & 0.209008 & 0.210284 & 0.2102773 & 0.00853409 & 0.008534 & 0.00853382 \\
\hline
\end{tabular}

Table 3 Frequency parameters of C-SD cylindrical shells $(h / R=0.002)$.

Frequency parameters $\Omega=\omega R \sqrt{\left(1-\mu^{2}\right) \rho / E}$

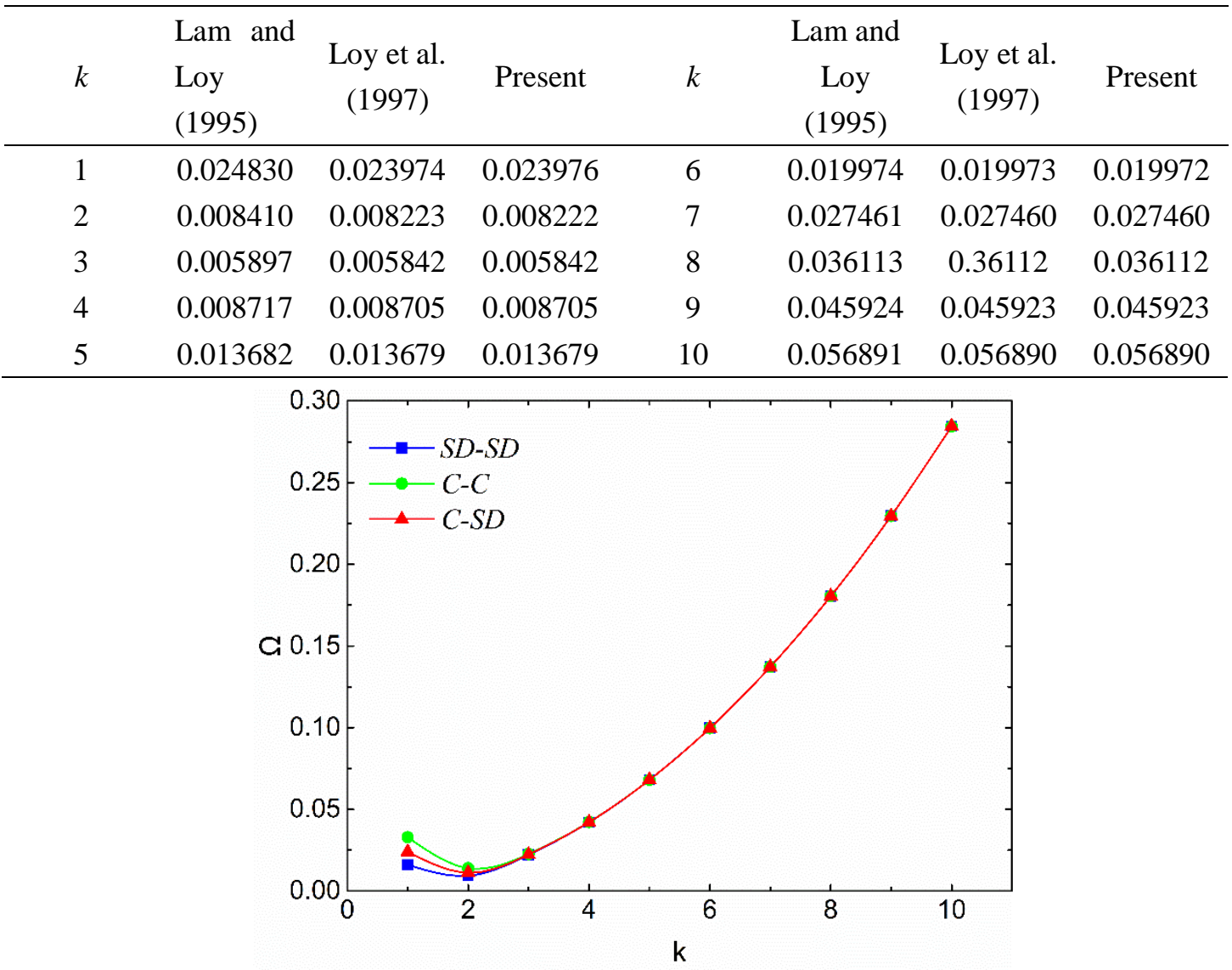

(a) different boundaries 


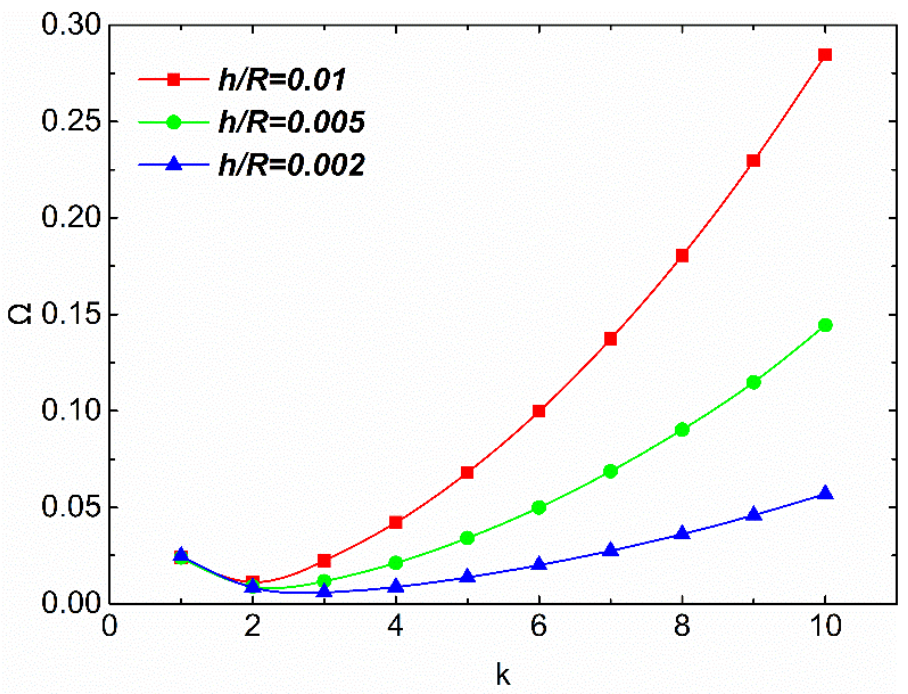

(b) different thickness-radius ratios $h / R$

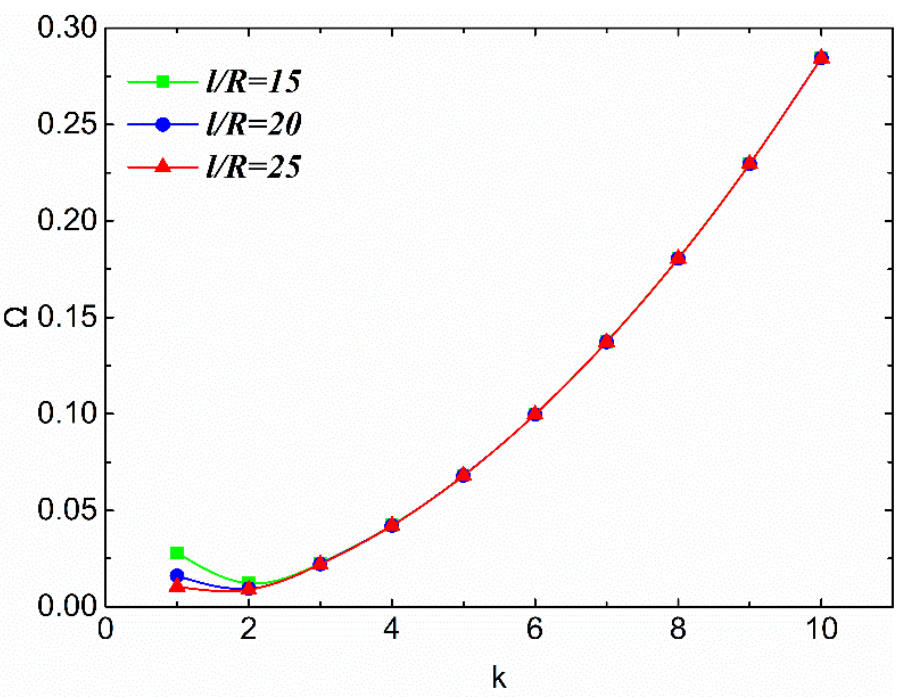

(c) different length-radius ratios $l / R$

Fig. 2. Variations of frequency parameters versus circumferential wavenumber $k$ : (a) different boundaries; (b) different thickness-radius ratios $h / R$.; (c) different length-radius ratios $l / R$.

Frequency parameters of a cylindrical shell with different boundaries, thickness-radius ratios, and length-radius ratios are plotted in Fig. 2. Circumferential wavenumbers ranging from 1 to 10 are considered here. As is shown this figure, with the increase of the circumferential wavenumber $k$, the general variation trend of frequency parameters decreases first and then increase. Frequency parameters for different boundaries coincide with each other when the circumferential wavenumber $k \geq 3$, and this indicates that the boundary type has a significant influence on frequency parameters corresponding to low circumferential wavenumbers. Furthermore, the frequency parameters increase with the increase of the thickness-radius ratios or decrease with the increase of the length-radius ratios. As can be seen from this figure, the effects of the thickness-radius ratios on the frequency parameters are mainly reflected in the case that the circumferential wavenumber $k \geq 2$, while the effects of the length-radius ratios on the frequency parameters are mainly reflected in the case that the 
circumferential wavenumber $\mathrm{k} \leq 3$.

\subsection{Convergence and validation}

To demonstrate the superiority of the developed approach used in this paper, the convergence of the developed approach is investigated with different number $N$ of grid points along the axial direction. A series of grid points, $N=5,7,9,11$ and 13, is used for this. Fig. 3 shows the influences of grid points on the time-history response of a cylindrical shell at a chosen position $(x=l / 2, \theta=0, z=0)$ and displacements of each point on the $x$-axis at a time when $t=0.26 \mathrm{~s}$. It is evident that the results tend to be stable and consistent when the number of grid points is higher than 7 , which suggests that the convergence rate of the developed approach used in the present work is fast. Moreover, in order to illustrate the merit of the proposed method in improving computation speed, the time required to use the developed method for calculating the dynamic response of the cylindrical shell is compared with the time required to adopt the Navier method. As is shown in Table 4, the time required to use the proposed method for calculating the dynamic response of the cylindrical shell is much less than the time required to the Navier method, and it indicates that the developed method is much more efficient than the Navier method in obtaining the dynamic response of the cylindrical shell.

Table 4 The time required to use different methods for calculating dynamic responses of the cylindrical shell.

\begin{tabular}{lccccc}
\hline \multicolumn{2}{c}{ Methods } & $1^{\text {st }}$ & $2^{\text {nd }}$ & $3^{\text {rd }}$ & Average time (s) \\
\hline NGP (The & 5 & 249.876 & 249.929 & 248.837 & 249.547 \\
present & 7 & 369.504 & 372.406 & 370.019 & 370.718 \\
method) & 9 & 665.313 & 662.661 & 668.121 & 665.365 \\
\hline \multicolumn{2}{r}{ The Navier method } & 1096.38 & 1089.37 & 1092.16 & 1092.64 \\
\hline
\end{tabular}

* NDP: the number of grid points

To prove the effectiveness of the present method, response results of cylindrical shells with SD-SD boundary are also compared with Navier's solution. The displacement components of a cylindrical shell with SD-SD boundary at the ends in terms of the Navier method can be expanded as follows:

$$
\left\{\begin{array}{l}
u(x, \theta, t)=\sum_{n=1}^{\infty} \sum_{m=1}^{\infty} u_{m n}(t) \cos \left(\frac{m \pi x}{l}\right) \cos (n \theta), \\
v(x, \theta, t)=\sum_{n=1}^{\infty} \sum_{m=1}^{\infty} v_{m n}(t) \sin \left(\frac{m \pi x}{l}\right) \sin (n \theta), \\
w(x, \theta, t)=\sum_{n=1}^{\infty} \sum_{m=1}^{\infty} w_{m n}(t) \sin \left(\frac{m \pi x}{l}\right) \cos (n \theta) .
\end{array}\right.
$$

where $u_{m n}(t), v_{m n}(t)$ and $w_{m n}(t)$ represent generalized coordinates, $m$ and $n$ denote the axial half wavenumber and circumferential wavenumber, respectively.

The results of the time-history response of the cylindrical shell with SD-SD boundary at a chosen position $(x=l / 2, \theta=0, z=0)$ obtained by the two methods are compared in Fig. 4 . As shown in this figure, the result derived from the semi-analytical method is in great agreement 
with the Navier's solution.
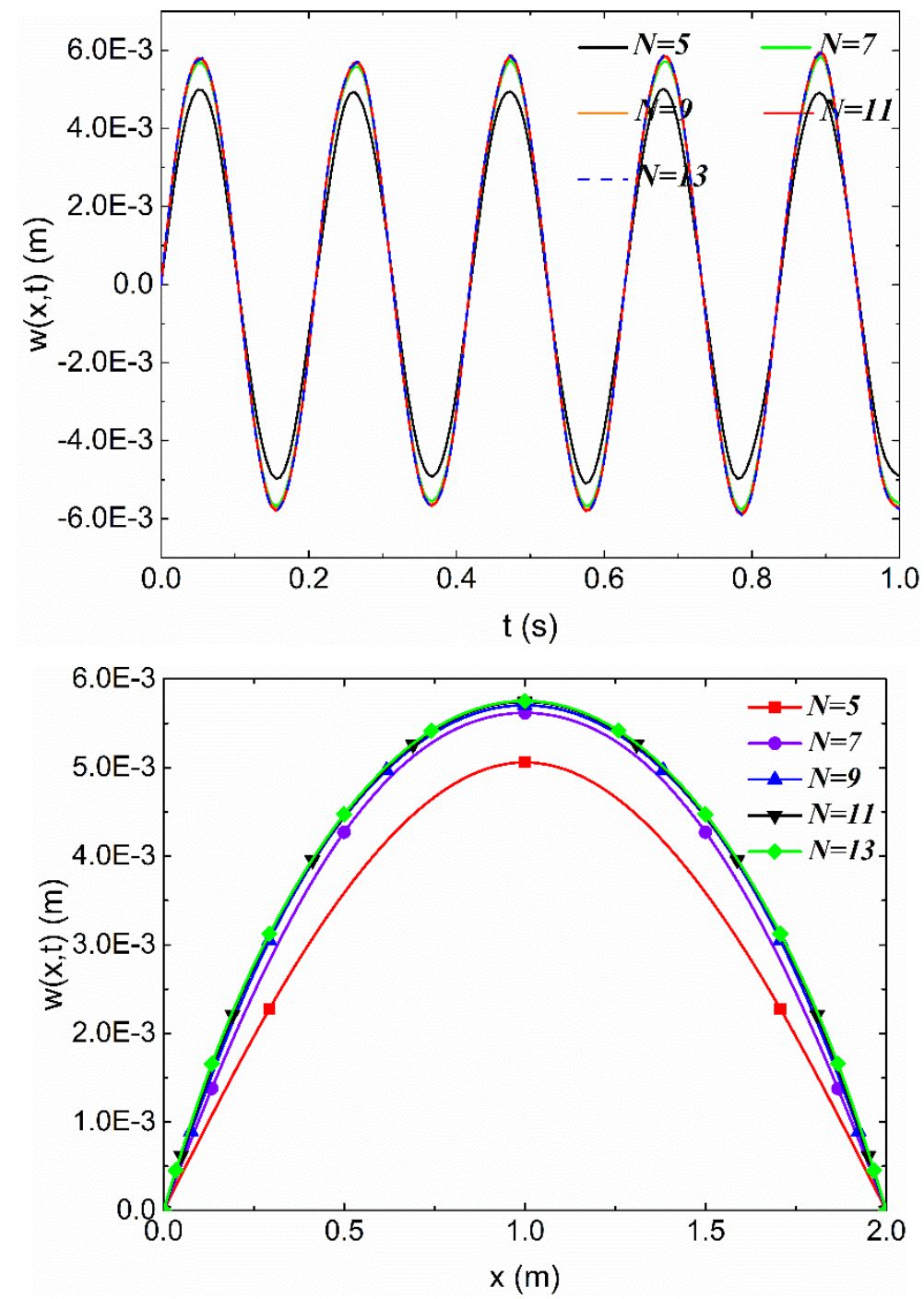

Fig. 3. The time-history curves of a SD-SD cylindrical shell at a chosen position $(x=l / 2, \theta=0$, $z=0)$ under different grid points and displacement of all grid points at a time when $t=0.26 \mathrm{~s}$.

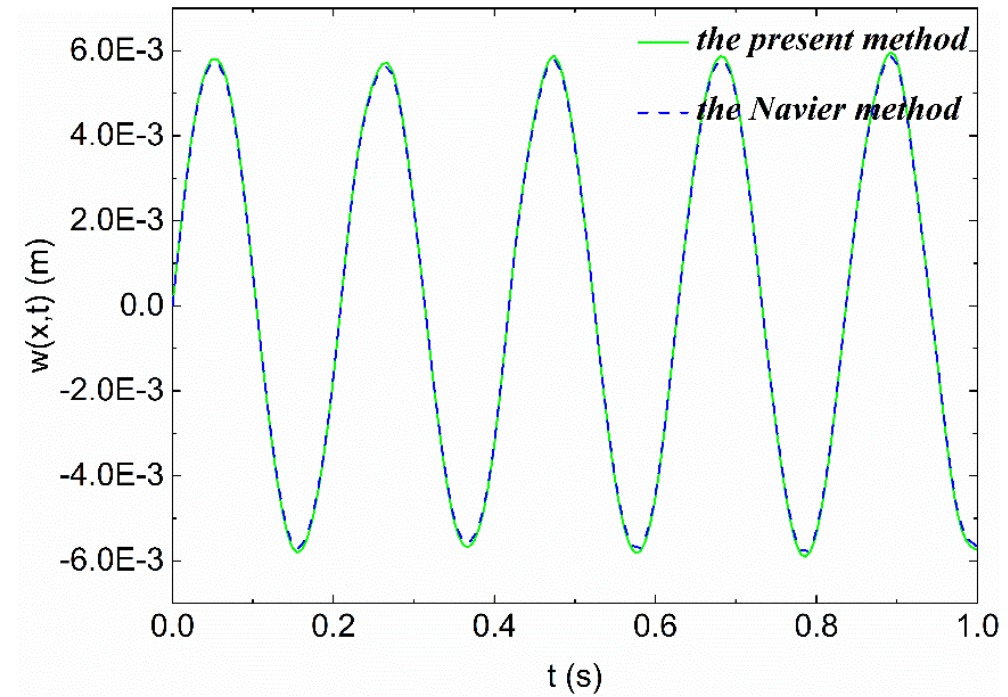

Fig. 4. The time-history curves of a SD-SD cylindrical shell at a chosen position $(x=l / 2, \theta=0$, $z=0)$ using the Navier method and the present method. 


\subsection{Comparison of different shell theories}

The influences of shell theories on the time-history responses of the cylindrical shell are analyzed. The results obtained by classical shell theory (CST) and first-order shear deformation theory (FSDT) with different boundaries and thickness-radius ratios are compared. Two sets of thickness-radius ratios $(h / R=1 / 500,1 / 1000)$ are used to present the time-history responses of cylindrical shells with SD-SD and C-C boundaries. As shown in Fig. 5, the results obtained by CST and FSDT under two sets of thickness-radius ratios are in good agreement, indicating that CST without considering shear deformation and rotary inertia is valid and accurate in calculating time-history responses of the cylindrical shell when the thickness-radius ratios are small enough.
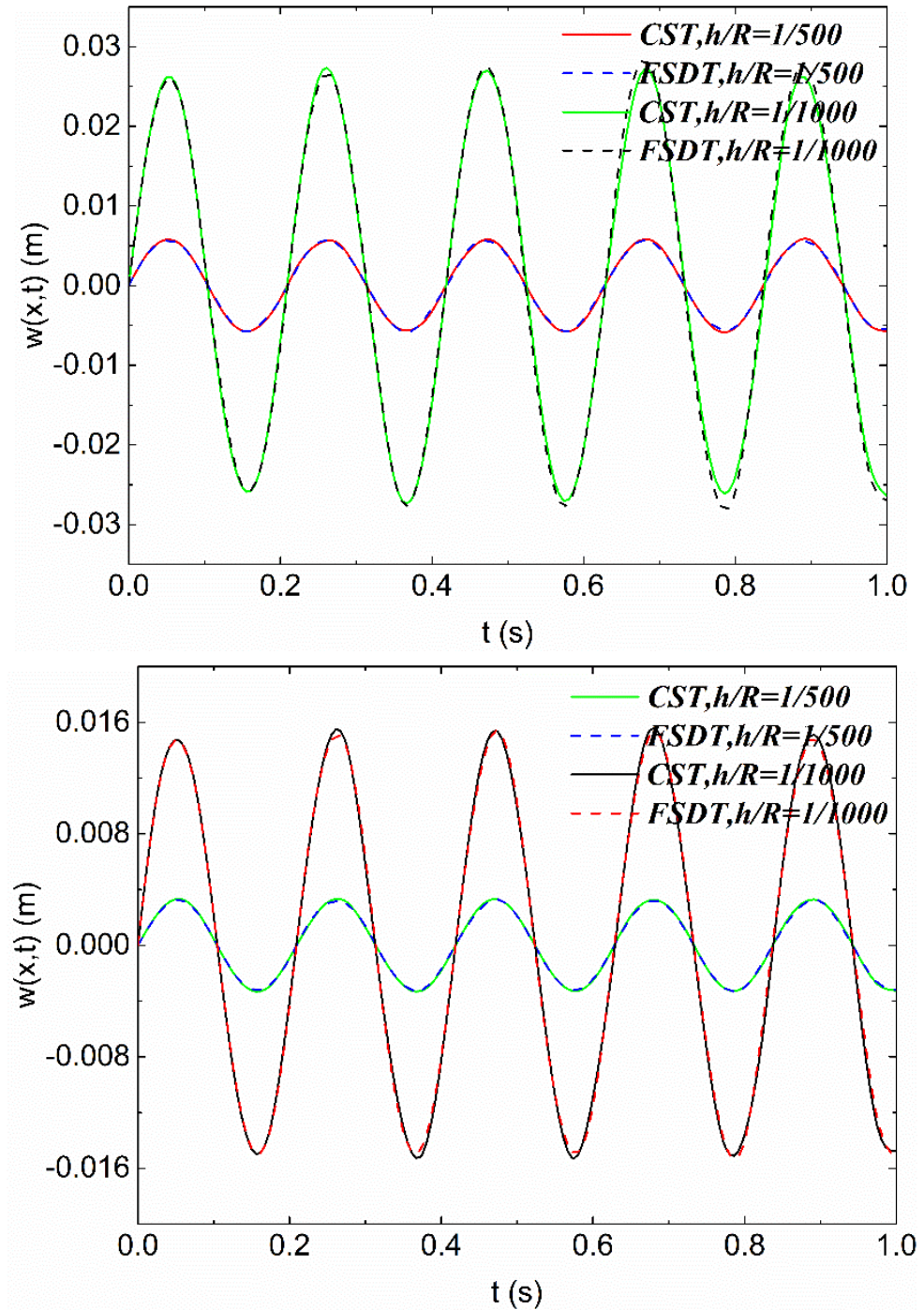

Fig. 5. The time-history curves of the cylindrical shell at a chosen position $(x=l / 2, \theta=0, z=0)$ under SD-SD and C-C boundaries.

\subsection{The influences of boundaries at the edges}

The influences of boundaries at the edges on the time-history response of the cylindrical shell are studied. Four kinds of boundaries at the edges are considered: Simply diaphragm-Simply diaphragm boundaries (SD-SD), Clamped-Clamped boundaries (C-C), Clamped-Simply diaphragm boundaries (C-SD) and Clamped-Free boundaries (C-F). The time-history responses of cylindrical shells under various boundaries at a chosen position 
$(x=l / 2, \theta=0, z=0)$ is plotted in Fig. 6. It is evident that the peak displacement is maximum when the boundary is C-F, and minimum peak displacement of the cylindrical shell appears when the boundary is $\mathrm{C}-\mathrm{C}$, and the results indicate that the peak displacement is closely related to the number of edge degrees of freedom.
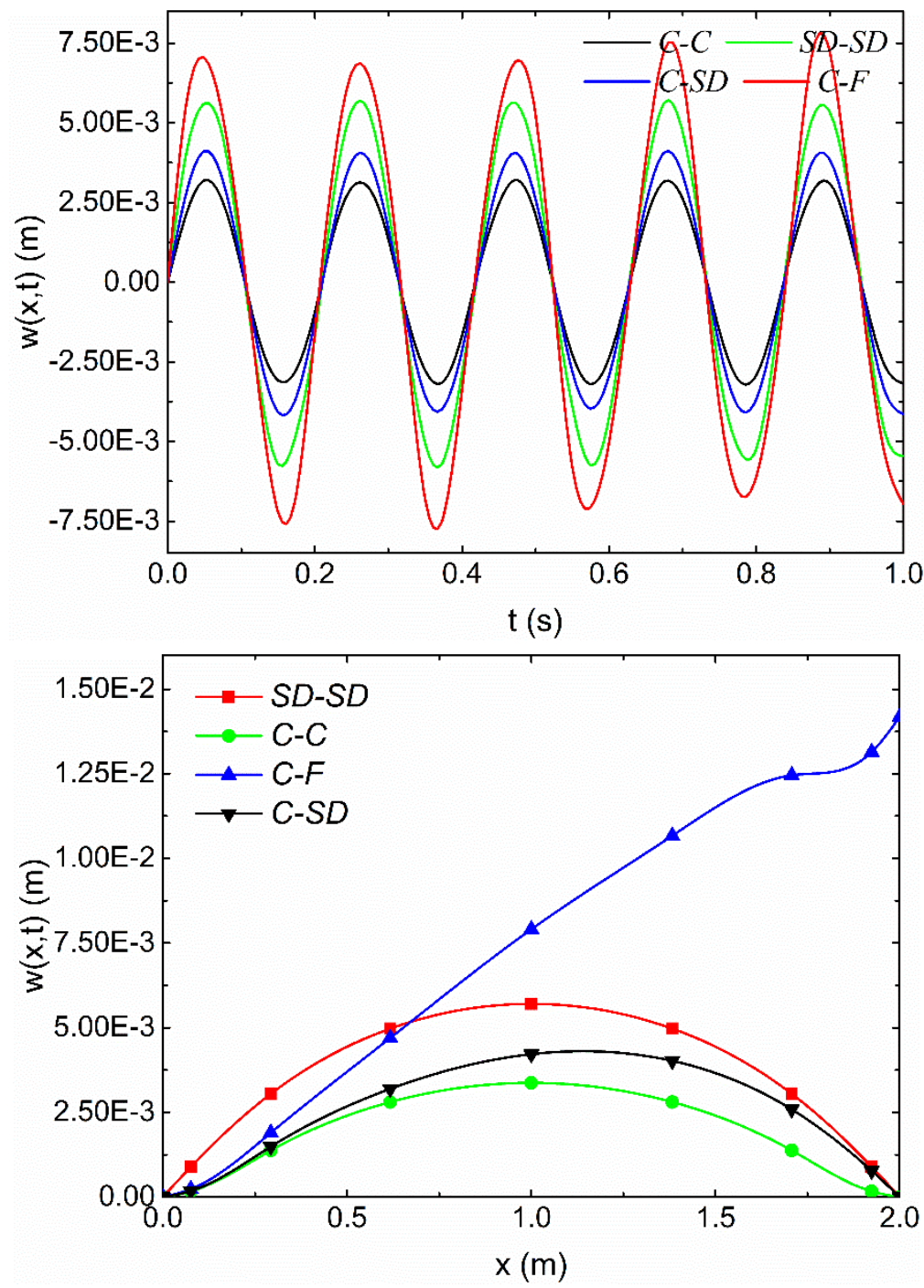

Fig. 6. The time-history curves of a cylindrical shell at a chosen position $(x=l / 2, \theta=0, z=0)$ under different boundaries and displacement of all grid points on the $x$-axis at a time when $t=0.26 \mathrm{~s}$.

\subsection{The influences of geometric dimensioning}

The influences of geometric dimensioning including length-radius ratio $l / R$ and thickness-radius ratio $h / R$ are evaluated. Two sets of geometric dimensioning are used, the first series is: $l / R=1,2,3,4,5$ with $h / R=1 / 250$; the second series is: $h / R=1 / 50,1 / 100,1 / 150$, $1 / 200,1 / 250$ with $l / R=4$. The time-history responses of the cylindrical shell for a given length-radius ratio and thickness-radius ratio at a chosen position $(x=l / 2, \theta=0, z=0)$ are given in Fig. 7. It is found that the peak displacement increases as length-radius ratio increases. On the contrary, for a fixed length-radius ratio, the peak displacement increases with a decrease in thickness-radius ratio. 

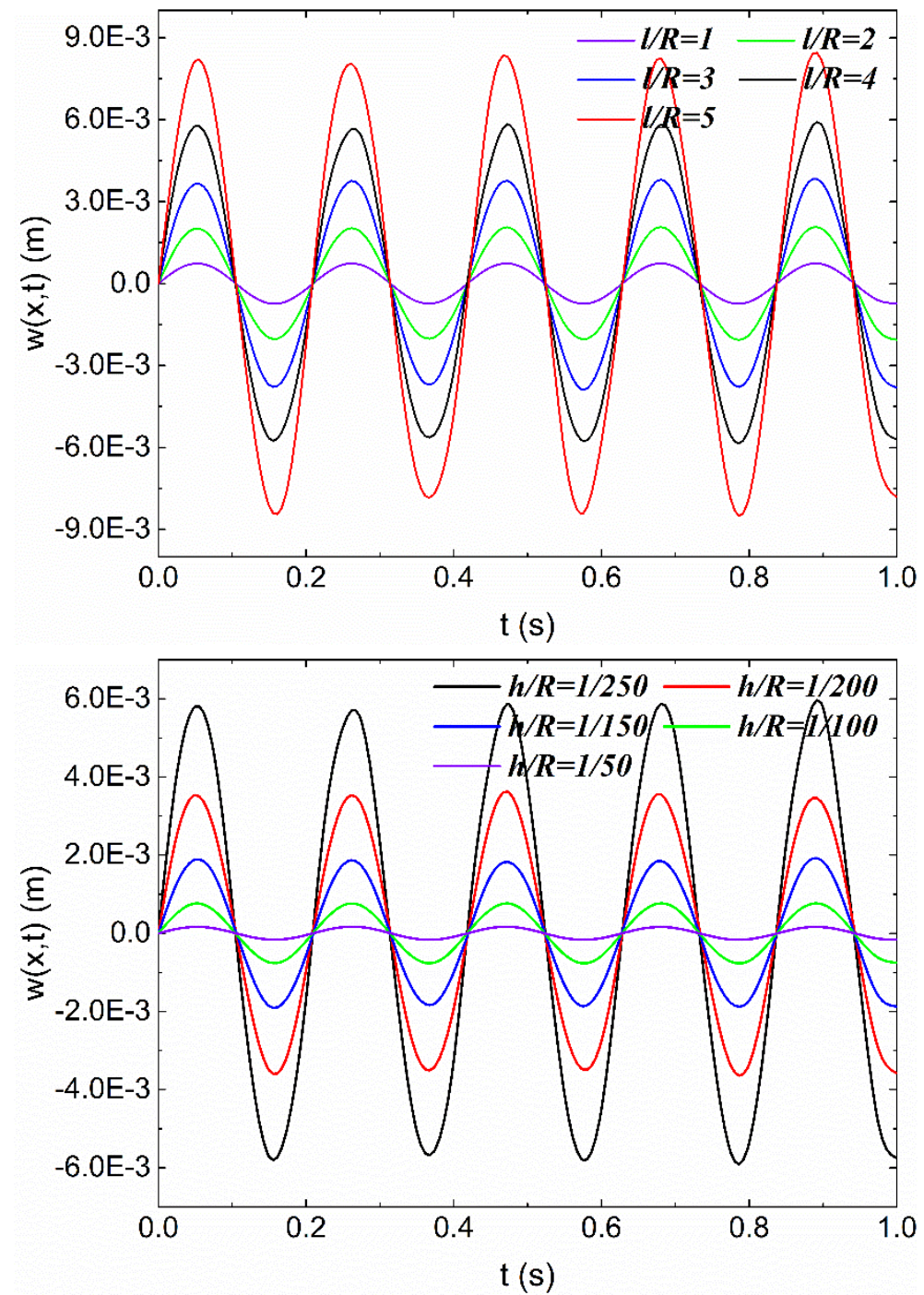

Fig. 7. The time-history responses of a cylindrical shell at a chosen position $(x=l / 2, \theta=0, z=0)$ under different length-radius ratios and thickness-radius ratios.

\section{Conclusions}

In the present work, a novel semi-analytical methodology, which includes the differential quadrature method, the Fourier series expansion technique, and Durbin's numerical inversion method, is proposed to derive the time-history responses of a cylindrical shell under various boundaries.

The frequency parameters of the thin cylindrical shells are calculated and a series of results obtained are compared with the data from the literature. This shows that the results obtained by the semi-analytical approach are consistent with the results in the literature. To demonstrate the convergence and the validity of the results obtained, varying numbers of grid points along the axial direction are used and a comparison of results with the Navier's solution is carried out. The results indicate that the approach used in this paper is of fast rapidity of convergence and high accuracy. A comparison of time-history responses derived by classical shell theory with results obtained by first-order shear deformation theory shows that the classical shell theory is accurate and precise in estimating the time-history responses of cylindrical shells when the thickness-radius ratio is small enough. The analysis of various 
boundaries on the time-history response of cylindrical shells shows that the peak displacement of cylindrical shells has a close relationship with the number of edge degrees of freedom. The influences of length-radius ratios and thickness-radius ratios on the time-history responses of cylindrical shells are also analyzed. Parametric studies show that the peak displacement becomes larger as the length-radius ratio increases or the thickness-radius ratio decreases.

\section{Acknowledgements}

This work was supported by the National Natural science Foundation of China (Grant Nos. 51879231, 51679214, 51409228), and the authors would like to express sincere gratitude to Victor Sun Zheng for his assistance in editing this manuscript.

\section{Appendix A}

$$
\begin{aligned}
& \mathbf{H}_{11}=A_{11} R \mathbf{A}_{i j}^{(2)}+\left(-\frac{A_{66} k^{2}}{R}-I_{0} R s^{2}\right) \mathbf{I}_{N}, \mathbf{H}_{12}=\left(A_{12} k+A_{66} k+\frac{B_{12} k}{R}+\frac{2 B_{66} k}{R}\right) \mathbf{A}_{i j}^{(1)}, \\
& \mathbf{H}_{13}=\left(A_{12}+\frac{B_{12} k^{2}}{R}+\frac{2 B_{66} k^{2}}{R}\right) \mathbf{A}_{i j}^{(1)}-B_{11} R \mathbf{A}_{i j}^{(3)}, \mathbf{H}_{21}=\left(-A_{12} k-A_{66} k-\frac{B_{12} k}{R}-\frac{2 B_{66} k}{R}\right) \mathbf{A}_{i j}^{(1)}, \\
& \mathbf{H}_{22}=\left(4 B_{66}+\frac{4 D_{66}}{R}+A_{66} R\right) \mathbf{A}_{i j}^{(2)}+\left(-\frac{D_{22} k^{2}}{R^{3}}-\frac{2 B_{22} k^{2}}{R^{2}}-\frac{A_{22} k^{2}}{R}-I_{0} R s^{2}\right) \mathbf{I}_{N}, \\
& \mathbf{H}_{23}=\left(B_{12} k+2 B_{66} k+\frac{D_{12} k}{R}+\frac{4 D_{66} k}{R}\right) \mathbf{A}_{i j}^{(2)}+\left(-\frac{D_{22} k^{3}}{R^{3}}-\frac{B_{22} k}{R^{2}}-\frac{B_{22} k^{3}}{R^{2}}-\frac{A_{22} k}{R}\right) \mathbf{I}_{N}, \\
& \mathbf{H}_{31}=\left(-A_{12}-\frac{B_{12} k^{2}}{R}-\frac{2 B_{66} k^{2}}{R}\right) \mathbf{A}_{i j}^{(1)}+B_{11} R \mathbf{A}_{i j}^{(3)}, \\
& \mathbf{H}_{32}=\left(B_{12} k+2 B_{66} k+\frac{D_{12} k}{R}+\frac{4 D_{66} k}{R}\right) \mathbf{A}_{i j}^{(2)}+\left(-\frac{D_{22} k^{3}}{R^{3}}-\frac{B_{22} k}{R^{2}}-\frac{B_{22} k^{3}}{R^{2}}-\frac{A_{22} k}{R}\right) \mathbf{I}_{N}, \\
& \mathbf{H}_{33}=\left(2 B_{12}+\frac{2 D_{12} k^{2}}{R}+\frac{4 D_{66} k^{2}}{R}\right) \mathbf{A}_{i j}^{(2)}-D_{11} R \mathbf{A}_{i j}^{(4)}+\left(-\frac{D_{22} k^{4}}{R^{3}}-\frac{2 B_{22} k^{2}}{R^{2}}-\frac{A_{22}}{R}-I_{0} R s^{2}\right) \mathbf{I}_{N} . \\
& I_{N}=\left[\begin{array}{cccc}
1 & 0 & \cdots & 0 \\
0 & 1 & \cdots & 0 \\
\vdots & \vdots & \ddots & \vdots \\
0 & 0 & \cdots & 1
\end{array}\right]_{N \times N}, \mathbf{A}_{i j}^{(m)}=\left[\begin{array}{cccc}
A_{11}^{(m)} & A_{12}^{(m)} & \cdots & A_{1 N}^{(m)} \\
A_{21}^{(m)} & A_{22}^{(m)} & \cdots & A_{2 N}^{(m)} \\
\vdots & \vdots & \ddots & \vdots \\
A_{N 1}^{(m)} & A_{N 2}^{(m)} & \cdots & A_{N N}^{(m)}
\end{array}\right] .
\end{aligned}
$$

\section{Appendix B}

$$
\begin{aligned}
& H_{11,1 i}=T_{11} A_{1 i}^{(1)}, i=2 \sim N, H_{11,11}=T_{11} A_{11}^{(1)}-k_{u 0}, \\
& H_{11, N i}=-T_{11} A_{N i}^{(1)}, i=1 \sim N-1, H_{11, N N}=-T_{11} A_{N N}^{(1)}-k_{u L}, \\
& H_{12,1 i}=0, i=2 \sim N, H_{12,11}=T_{12}, \\
& H_{12, N i}=0, i=1 \sim N-1, H_{12, N N}=-T_{12}, \\
& H_{13,1 i}=0, i=2 \sim N, H_{13,11}=T_{13}, \\
& H_{13, N i}=0, i=1 \sim N-1, H_{13, N N}=-T_{13} .
\end{aligned}
$$




$$
\begin{aligned}
& H_{21,1 i}=0, i=2 \sim N, H_{21,11}=T_{21}, \\
& H_{21, N i}=0, i=1 \sim N-1, H_{21, N N}=-T_{21}, \\
& H_{22,1 i}=T_{22} A_{1 i}^{(1)}, i=2 \sim N, H_{22,11}=T_{22} A_{11}^{(1)}-k_{v 0}, \\
& H_{22, N i}=-T_{22} A_{N i}^{(1)}, i=1 \sim N-1, H_{22, N N}=-T_{22} A_{N N}^{(1)}-k_{v L}, \\
& H_{23,1 i}=T_{23} A_{1 i}^{(1)}, H_{23, N i}=-T_{23} A_{N i}^{(1)}, i=1 \sim N . \\
& H_{31,1 i}=0, H_{31, N i}=0, i=1 \sim N, \\
& H_{32,1 i}=T_{32} A_{1 i}^{(1)}, i=2 \sim N-1, H_{32,11}=T_{32} A_{11}^{(1)}, \\
& H_{32, N i}=-T_{32} A_{N i}^{(1)}, i=1 \sim N-1, H_{32, N N}=-T_{32} A_{N N}^{(1)}, \\
& H_{33,1 i}=T_{331} A_{1 i}^{(1)}+T_{332} A_{1 i}^{(3)}, i=2 \sim N, \\
& H_{33,11}=T_{331} A_{11}^{(1)}+T_{332} A_{11}^{(3)}-k_{w 0}, \\
& H_{33, N i}=-T_{331} A_{N i}^{(1)}-T_{332} A_{N i}^{(3)}, i=1 \sim N-1, \\
& H_{33, N N}=-T_{331} A_{N N}^{(1)}-T_{332} A_{N N}^{(3)}-k_{w L} . \\
& H_{31,2 i}=0, H_{31, N-1, i}=0, i=1 \sim N, \\
& H_{32,2 i}=0, i=2 \sim N-1, H_{32,21}=T_{42}, \\
& H_{32, N-1, i}=0, i=1 \sim N-1, H_{32, N-1, N}=-T_{42}, \\
& H_{33,2 i}=T_{432} A_{1 i}^{(2)}-K_{w 0} A_{1 i}^{(1)}, i=2 \sim N, \\
& H_{33,21}=T_{432} A_{11}^{(2)}-K_{w 0} A_{11}^{(1)}+T_{431}, \\
& H_{33, N-1, i}=-T_{432} A_{N i}^{(2)}-K_{w 0} A_{N i}^{(1)}, i=1 \sim N-1, \\
& H_{33, N-1, N}=-T_{432} A_{N N}^{(2)}-K_{w 0} A_{N N}^{(1)}-T_{431} .
\end{aligned}
$$

\section{References}

Bodaghi, M., Shakeri, M., 2012. An analytical approach for free vibration and transient response of functionally graded piezoelectric cylindrical panels subjected to impulsive loads. Composite Structures 94 (5), 1721-1735.

Civalek, Ö., 2006. The determination of frequencies of laminated conical shells via the discrete singular convolution method. Journal of Mechanics of Materials \& Structures 1 (1), 163-182.

Civalek, Ö., 2013. Vibration analysis of laminated composite conical shells by the method of discrete singular convolution based on the shear deformation theory. Composites Part B Engineering 45 (1), 1001-1009.

Duc, N.D., 2013. Nonlinear dynamic response of imperfect eccentrically stiffened FGM double curved shallow shells on elastic foundation. Composite Structures 99, 88-96.

Durbin, F., 1974. Numerical inversion of Laplace Transforms: An efficient improvement to Dubner and Abate's method. Computer Journal 17 (4), 371-376.

Ferreira, A.J.M., Carrera, E., Cinefra, M., Roque, C.M.C., Polit, O., 2011. Analysis of laminated shells by a sinusoidal shear deformation theory and radial basis functions collocation, accounting for through-the-thickness deformations. Composites Part B Engineering 42 (5), 1276-1284.

Ferreira, A.J.M., Roque, C.M.C., Jorge, R.M.N., 2006. Modelling cross-ply laminated elastic shells by a higher-order theory and multiquadrics. Computers \& Structures 84 (19), 
1288-1299.

Frikha, A., Zghal, S., Dammak, F., 2018. Dynamic analysis of functionally graded carbon nanotubes-reinforced plate and shell structures using a double directors finite shell element. Aerospace Science \& Technology 78, 438-451.

Gu, J., An, C., Duan, M., Levi, C., Su, J., 2013. Integral transform solutions of dynamic response of a clamped-clamped pipe conveying fluid. Nuclear Engineering \& Design 254 (1), 237-245.

Gupta, S., Matos, H., Leblanc, J.M., Shukla, A., 2016. Shock initiated instabilities in underwater cylindrical structures. Journal of the Mechanics \& Physics of Solids 95, 188-212. Haddadpour, H., Mahmoudkhani, S., Navazi, H.M., 2007. Free vibration analysis of functionally graded cylindrical shells including thermal effects. Thin-Walled Structures 45 (6), 591-599.

Hajmohammad, M.H., Kolahchi, R., Zarei, M.S., Maleki, M., 2017. Earthquake induced dynamic deflection of submerged viscoelastic cylindrical shell reinforced by agglomerated CNTs considering thermal and moisture effects. Composite Structures 187, 498-508.

Jin, G., Yang, C., Liu, Z., Gao, S., Zhang, C., 2015. A unified method for the vibration and damping analysis of constrained layer damping cylindrical shells with arbitrary boundary conditions. Composite Structures 130, 124-142.

Jin, G., Ye, T., Ma, X., Chen, Y., Su, Z., Xie, X., 2013. A unified approach for the vibration analysis of moderately thick composite laminated cylindrical shells with arbitrary boundary conditions. International Journal of Mechanical Sciences 75 (10), 357-376.

Kadoli, R., Ganesan, N., 2006. Buckling and free vibration analysis of functionally graded cylindrical shells subjected to a temperature-specified boundary condition. Journal of Sound \& Vibration 289 (3), 450-480.

Lam, K.Y., Loy, C.T., 1995. Effects of boundary conditions on frequencies of a multi-layered cylindrical shell. Journal of Sound \& Vibration 188 (188), 363-384.

Leissa, A.W., 1993. Vibration of shells. US Government Printing Office, Washington DC.

Liang, S., Chen, H.L., 2006. The natural vibration of a conical shell with an annular end plate. Journal of Sound \& Vibration 294 (4), 927-943.

Liang, X., Wang, Z., Wang, L., Liu, G., 2014. Semi-analytical solution for three-dimensional transient response of functionally graded annular plate on a two parameter viscoelastic foundation. Journal of Sound \& Vibration 333 (12), 2649-2663.

Liu, J., He, W., Xie, D., 2018. Study on Vibrational Power Flow Propagation Characteristics in a Laminated Composite Cylindrical Shell Filled with Fluid. Shock \& Vibration 2018 (4), $1-19$.

Loy, C.T., Lam, K.Y., Shu, C., 1997. Analysis of cylindrical shells using generalized differential quadrature. Shock \& Vibration 4 (3), 193-198.

Lu, C., Lam, K.Y., 1995. Dynamic analysis of clamped laminated curved panels. Composite Structures 30 (4), 389-398.

Maleki, S., Tahani, M., Andakhshideh, A., 2012. Static and transient analysis of laminated cylindrical shell panels with various boundary conditions and general lay - ups. ZAMM Journal of Applied Mathematics and Mechanics / Zeitschrift für Angewandte Mathematik und Mechanik 92 (2), 124-140.

Markuš, Š., 1988. The mechanics of vibrations of cylidrical shells. Elsevier, New York. 
Pradhan, S.C., Loy, C.T., Lam, K.Y., Reddy, J.N., 2000. Vibration characteristics of functionally graded cylindrical shells under various boundary conditions. Applied Acoustics 61 (1), 111-129.

Qatu, M.S., 2004. Vibration of laminated shells and plates. Elsevier, San Diego.

Qing, G., Liu, Y., Guo, Q., Zhang, D., 2008. Dynamic analysis for three-dimensional laminated plates and panels with damping. International Journal of Mechanical Sciences 50 (1), 83-91.

Qu, Y., Hua, H., Meng, G., 2013a. A domain decomposition approach for vibration analysis of isotropic and composite cylindrical shells with arbitrary boundaries. Composite Structures 95, 307-321.

Qu, Y., Long, X., Wu, S., Meng, G., 2013b. A unified formulation for vibration analysis of composite laminated shells of revolution including shear deformation and rotary inertia. Composite Structures 98 (3), 169-191.

Reddy, J.N., 2003. Mechanics of laminated composite plates and shells : theory and analysis. CRC Press, Florida.

Reddy, J.N., Khdeir, A.A., 1989. Dynamic response of cross - ply laminated shallow shells according to a refined shear deformation theory. Journal of the Acoustical Society of America 85 (6), 2423-2431.

Santos, H., Soares, C.M.M., Soares, C.A.M., Reddy, J.N., 2009. A semi-analytical finite element model for the analysis of cylindrical shells made of functionally graded materials. Composite Structures 91 (4), 427-432.

Shao, D., Hu, S., Wang, Q., Pang, F., 2017a. An enhanced reverberation-ray matrix approach for transient response analysis of composite laminated shallow shells with general boundary conditions. Composite Structures 162, 133-155.

Shao, D., Wang, Q., Qin, B., 2017b. A simple first-order shear deformation shell theory for vibration analysis of composite laminated open cylindrical shells with general boundary conditions. Composite Structures.

Thai, H.T., Kim, S.E., 2015. A review of theories for the modeling and analysis of functionally graded plates and shells. Composite Structures 128 (3), 70-86.

Wu, J., Chong, J., Long, Y., Zhou, Y., Yu, Y., Liu, J., 2018. Experimental study on the deformation and damage of cylindrical shell-water-cylindrical shell structures subjected to underwater explosion. Thin-Walled Structures 127, 654-665.

Yazdi, A.A., 2013. Applicability of homotopy perturbation method to study the nonlinear vibration of doubly curved cross-ply shells. Composite Structures 96 (96), 526-531.

Zhang, L.W., Song, Z.G., Qiao, P., Liew, K.M., 2017. Modeling of dynamic responses of CNT-reinforced composite cylindrical shells under impact loads. Computer Methods in Applied Mechanics \& Engineering 313, 889-903.

Zhang, Y., Duan, M., Yi, W., Gui, C., 2015a. Analytical study of the strength of adhesive joints of riser pipes. Ships \& Offshore Structures 10 (5), 545-553.

Zhang, Y., Wu, P., Duan, M., 2015b. A mesh-independent technique to evaluate stress singularities in adhesive joints. International Journal of Adhesion \& Adhesives 57, 105-117. 


\section{List of Tables}

Table 1 Frequency parameters of a thin cylindrical shell with three different boundaries $(h / R=0.01)$.

Table 2 Frequency parameters of SD-SD cylindrical shells.

Table 3 Frequency parameters of C-SD cylindrical shells $(h / R=0.002)$.

Table 4 The time required to use different methods for calculating dynamic responses of the cylindrical shell. 
Table 1 Frequency parameters of a thin cylindrical shell with three different boundaries $(h / R=0.01)$.

\begin{tabular}{ccccccc}
\hline & \multicolumn{5}{c}{ Frequency parameters $\Omega=\omega R \sqrt{\left(1-\mu^{2}\right) \rho / E}$} \\
\cline { 2 - 7 }$k$ & \multicolumn{2}{c}{ SD-SD } & \multicolumn{3}{c}{ C-C } & \multicolumn{3}{c}{ C-SD } \\
\cline { 2 - 7 } & $\begin{array}{c}\text { Loy et al. } \\
(1997)\end{array}$ & Present & $\begin{array}{c}\text { Loy et al. } \\
(1997)\end{array}$ & Present & $\begin{array}{c}\text { Loy et al. } \\
(1997)\end{array}$ & Present \\
\hline 1 & 0.016101 & 0.016101 & 0.032885 & 0.032884 & 0.023974 & 0.023976 \\
2 & 0.009382 & 0.009378 & 0.013923 & 0.013929 & 0.011225 & 0.011221 \\
3 & 0.022105 & 0.022103 & 0.022672 & 0.022668 & 0.022310 & 0.022308 \\
4 & 0.042095 & 0.042094 & 0.042208 & 0.042207 & 0.042139 & 0.042137 \\
5 & 0.068008 & 0.068007 & 0.068046 & 0.068045 & 0.068024 & 0.068023 \\
6 & 0.099730 & 0.099729 & 0.099748 & 0.099747 & 0.099738 & 0.099737 \\
7 & 0.137239 & 0.137238 & 0.137249 & 0.137249 & 0.137244 & 0.137243 \\
8 & 0.180527 & 0.180527 & 0.180535 & 0.180534 & 0.180531 & 0.180531 \\
9 & 0.229594 & 0.229593 & 0.229599 & 0.229598 & 0.229596 & 0.229596 \\
10 & 0.284435 & 0.284435 & 0.284439 & 0.284439 & 0.284437 & 0.284437 \\
\hline
\end{tabular}


Table 2 Frequency parameters of SD-SD cylindrical shells.

\begin{tabular}{ccccccc}
\hline \multicolumn{8}{c}{ Frequency parameters $\Omega=\omega R \sqrt{\left(1-\mu^{2}\right) \rho / E}$} \\
\hline \multirow{3}{*}{$k$} & \multicolumn{3}{c}{$h / R=0.05$} & & \multicolumn{3}{c}{$h / R=0.002$} \\
\cline { 2 - 7 } & Markuš & Loy et al. & Present & Markuš & Loy et al. & Present \\
\hline 1 & 0.0161063 & 0.01610 & 0.0161029 & 0.0161011 & 0.016101 & 0.0161011 \\
2 & 0.0392332 & 0.03930 & 0.0392710 & 0.00545243 & 0.005453 & 0.00545297 \\
3 & 0.109477 & 0.109824 & 0.1098116 & 0.00503724 & 0.005042 & 0.00504148 \\
4 & 0.209008 & 0.210284 & 0.2102773 & 0.00853409 & 0.008534 & 0.00853382 \\
\hline
\end{tabular}


Table 3 Frequency parameters of C-SD cylindrical shells $(h / R=0.002)$.

Frequency parameters $\Omega=\omega R \sqrt{\left(1-\mu^{2}\right) \rho / E}$

\begin{tabular}{llllccccc}
\hline & $\begin{array}{l}\text { Lam and } \\
k\end{array}$ & Loy \\
$(1995)$ & $\begin{array}{c}\text { Loy et al. } \\
(1997)\end{array}$ & Present & $k$ & $\begin{array}{c}\text { Lam and } \\
\text { Loy }\end{array}$ & $\begin{array}{c}\text { Loy et al. } \\
(1997)\end{array}$ & Present \\
& & & & $(1995)$ & \\
\hline 1 & 0.024830 & 0.023974 & 0.023976 & 6 & 0.019974 & 0.019973 & 0.019972 \\
2 & 0.008410 & 0.008223 & 0.008222 & 7 & 0.027461 & 0.027460 & 0.027460 \\
3 & 0.005897 & 0.005842 & 0.005842 & 8 & 0.036113 & 0.36112 & 0.036112 \\
4 & 0.008717 & 0.008705 & 0.008705 & 9 & 0.045924 & 0.045923 & 0.045923 \\
5 & 0.013682 & 0.013679 & 0.013679 & 10 & 0.056891 & 0.056890 & 0.056890 \\
\hline
\end{tabular}


Table 4 The time required to use different methods for calculating dynamic responses of the cylindrical shell.

\begin{tabular}{lccccc}
\hline \multicolumn{2}{c}{ Methods } & $1^{\text {st }}$ & $2^{\text {nd }}$ & $3^{\text {rd }}$ & Average time (s) \\
\hline NGP (The & 5 & 249.876 & 249.929 & 248.837 & 249.547 \\
present & 7 & 369.504 & 372.406 & 370.019 & 370.718 \\
method) & 9 & 665.313 & 662.661 & 668.121 & 665.365 \\
\hline \multicolumn{2}{r}{ The Navier method } & 1096.38 & 1089.37 & 1092.16 & 1092.64 \\
\hline
\end{tabular}

* NDP: the number of grid points 


\section{List of Figures}

Fig. 1. Geometry of the thin cylindrical shell subjected to a harmonic load.

Fig. 2. Variations of frequency parameters versus circumferential wavenumber $k$ : (a) different boundaries; (b) different thickness-radius ratios $h / R$; (c) different length-radius ratios $l / R$.

Fig. 3. The time-history curves of a SD-SD cylindrical shell at a chosen position $(x=l / 2, \theta=0$, $z=0)$ under different grid points and displacement of all grid points at a time when $t=0.26 \mathrm{~s}$.

Fig. 4 . The time-history curves of a SD-SD cylindrical shell at a chosen position $(x=l / 2, \theta=0$, $z=0$ ) using the Navier method and the present method.

Fig. 5. The time-history curves of the cylindrical shell at a chosen position $(x=l / 2, \theta=0, z=0)$ under SD-SD and C-C boundaries.

Fig. 6. The time-history curves of a cylindrical shell at a chosen position $(x=l / 2, \theta=0, z=0)$ under different boundaries and displacement of all grid points on the $x$-axis at a time when $t=0.26 \mathrm{~s}$.

Fig. 7. The time-history responses of a cylindrical shell at a chosen position $(x=l / 2, \theta=0, z=0)$ under different length-radius ratios and thickness-radius ratios. 
1

2

3

4

5

6

7

8

10

11

12

13

14

15

16

17

18

19

20

21

22

23

24

25

26

27

28

29

30

31

32

33

34

35

36

37

38

39

40

41

42

43

44

45

46

47

48

49

50

51

52

53

54

55

56

57

58

59

60

61

62

63

64

65

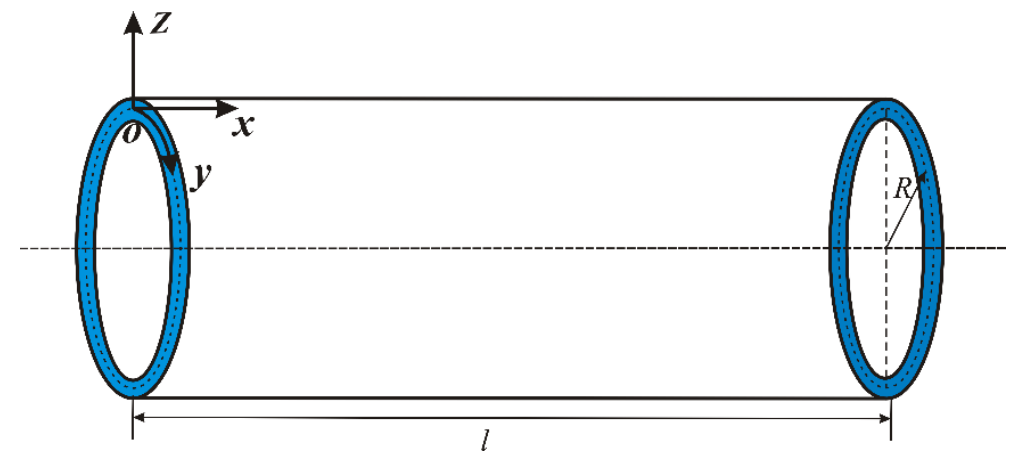

Fig. 1. Geometry of the thin cylindrical shell subjected to a harmonic load. 


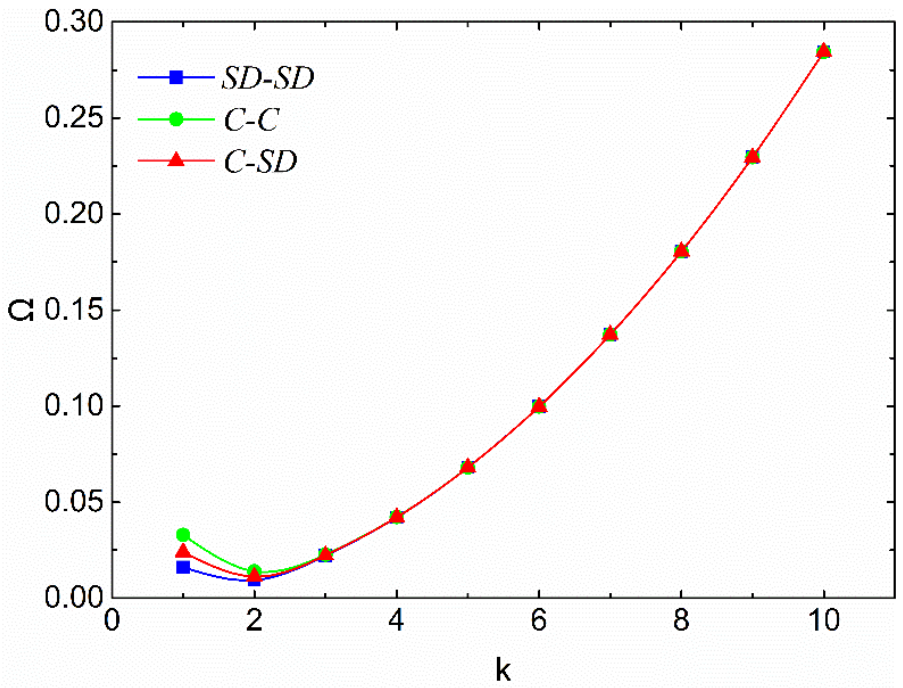

(a) different boundaries

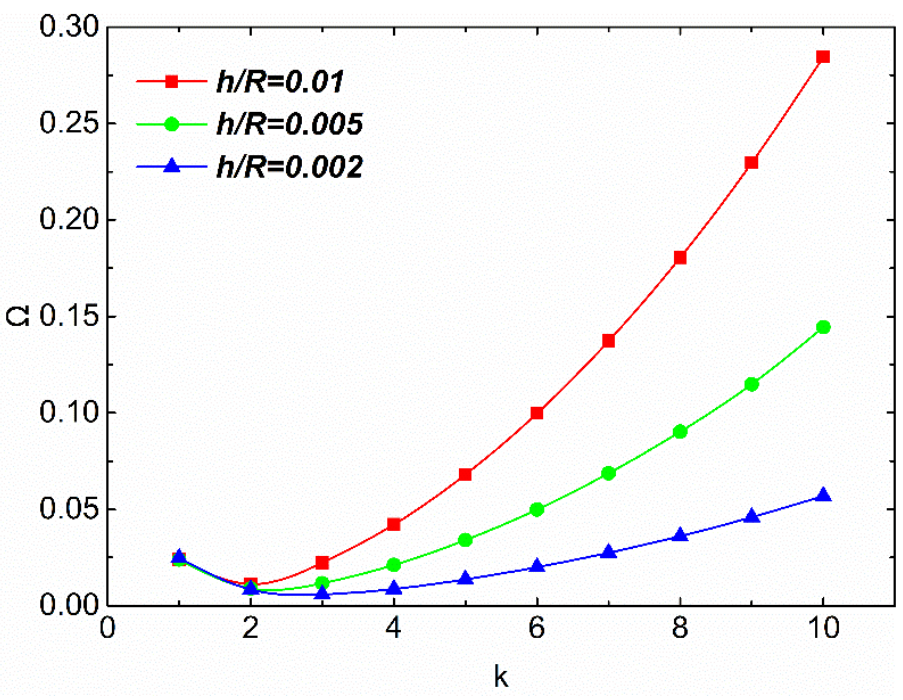

(b) different thickness-radius ratios $h / R$

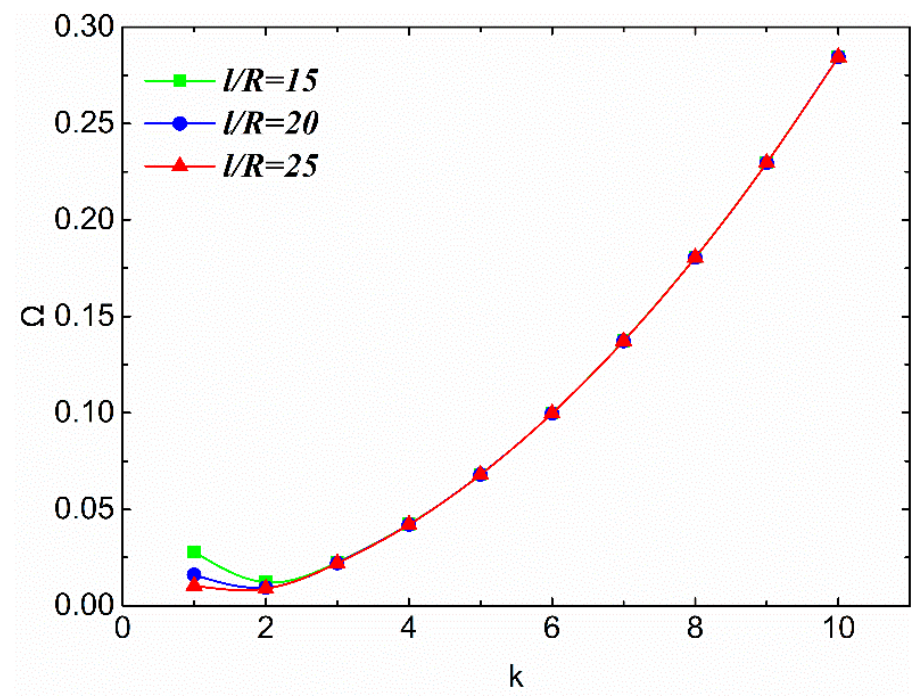

(c) different length-radius ratios $l / R$

Fig. 2. Variations of frequency parameters versus circumferential wavenumber $k$ : (a) different boundaries; (b) different thickness-radius ratios $h / R$.; (c) different length-radius ratios $l / R$. 

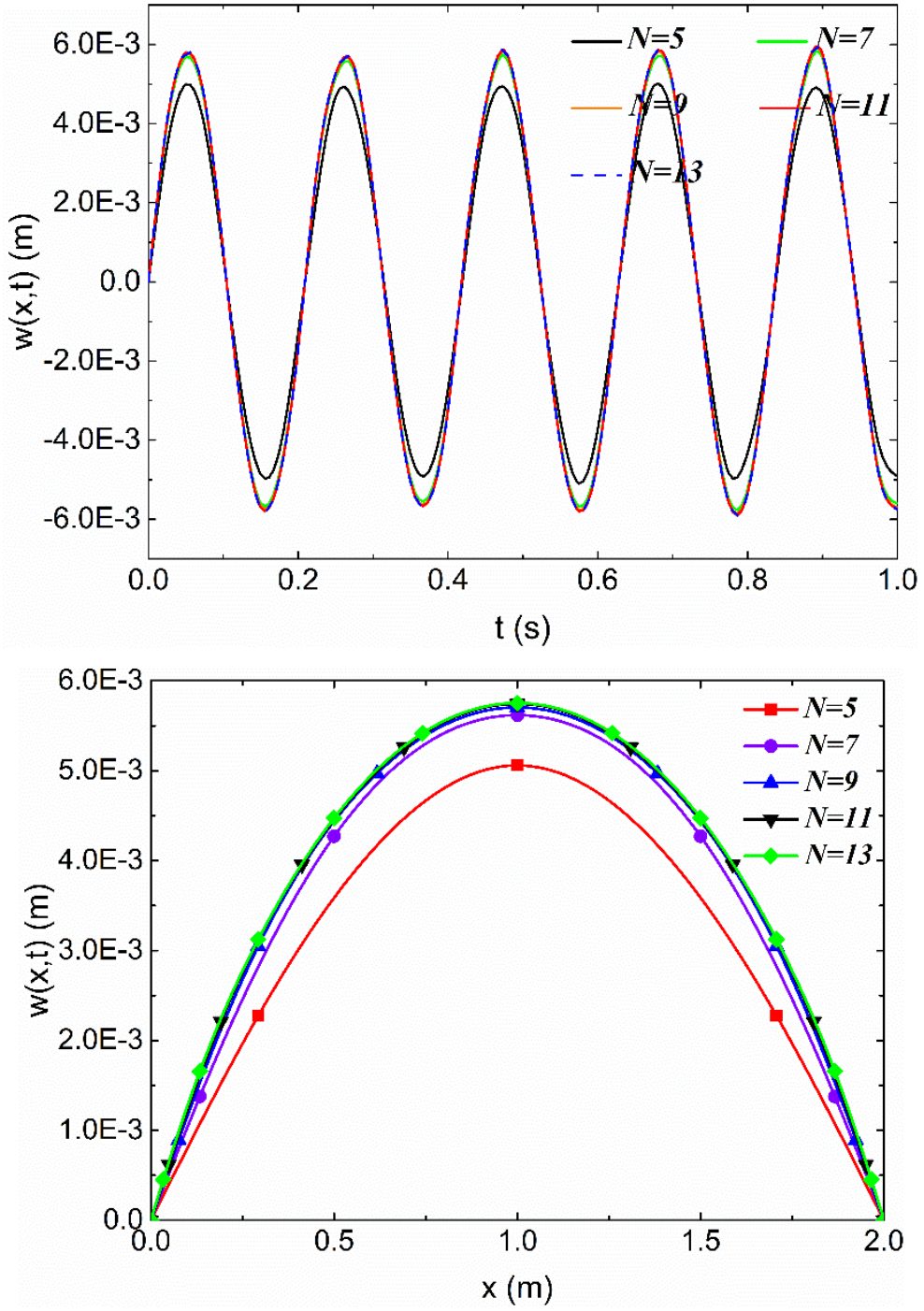

Fig. 3. The time-history curves of a SD-SD cylindrical shell at a chosen position $(x=l / 2, \theta=0$, $z=0)$ under different grid points and displacement of all grid points at a time when $t=0.26 \mathrm{~s}$. 


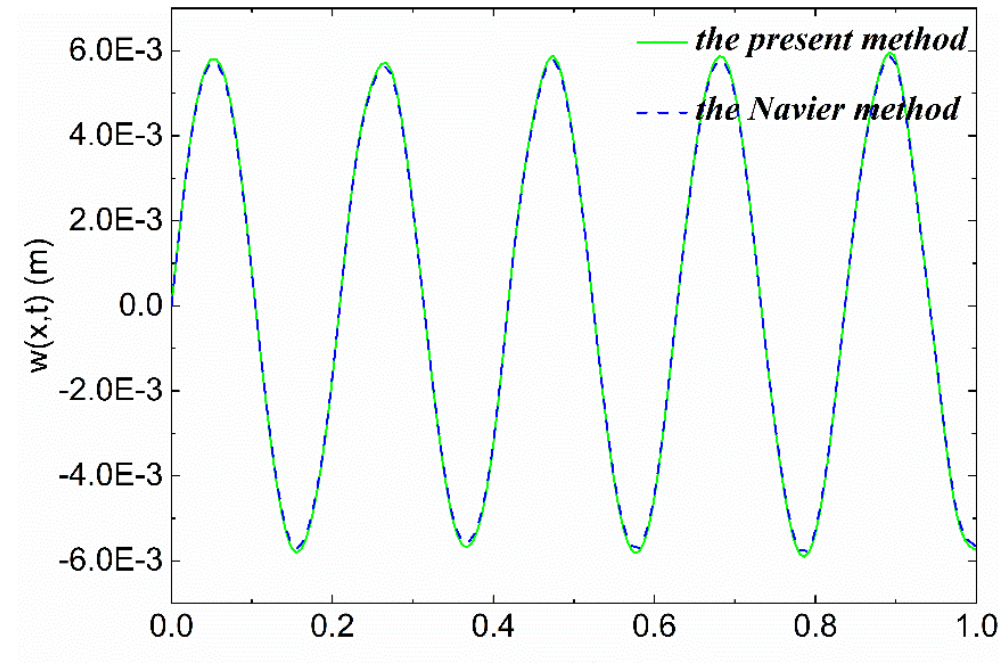

$t(s)$

Fig. 4. The time-history curves of a SD-SD cylindrical shell at a chosen position $(x=l / 2, \theta=0$, $z=0$ ) using the Navier method and the present method. 


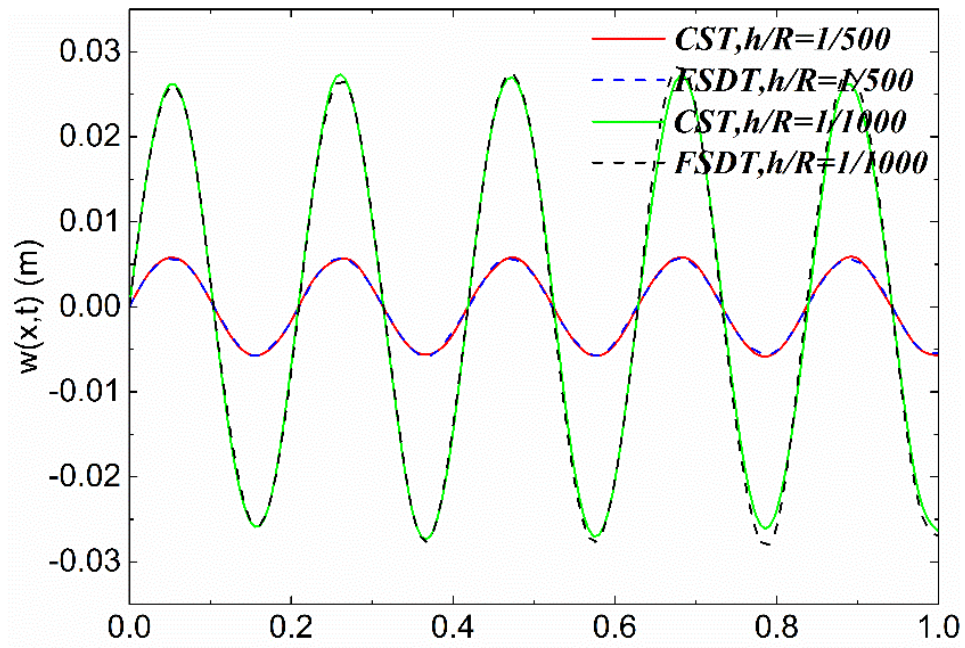

$t$ (s)

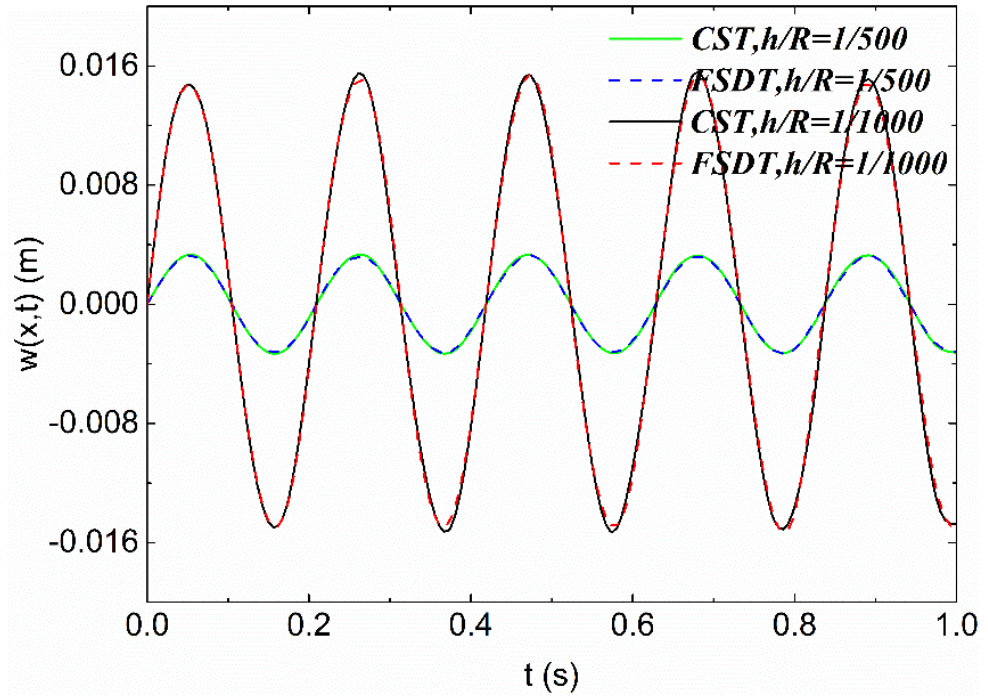

Fig. 5. The time-history curves of the cylindrical shell at a chosen position $(x=l / 2, \theta=0, z=0)$ under SD-SD and C-C boundaries. 

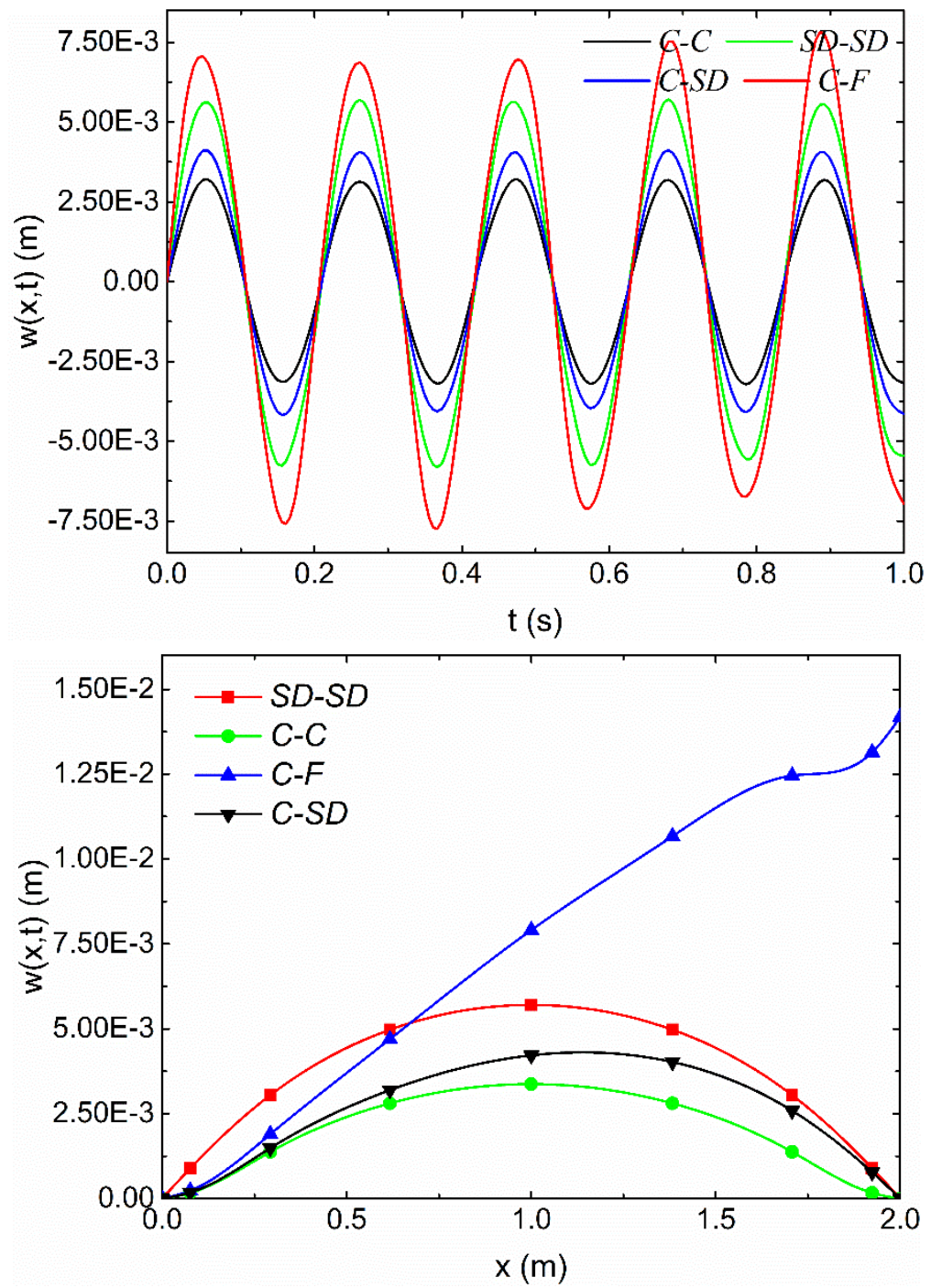

Fig. 6. The time-history curves of a cylindrical shell at a chosen position $(x=l / 2, \theta=0, z=0)$ under different boundaries and displacement of all grid points on the $x$-axis at a time when $t=0.26 \mathrm{~s}$. 

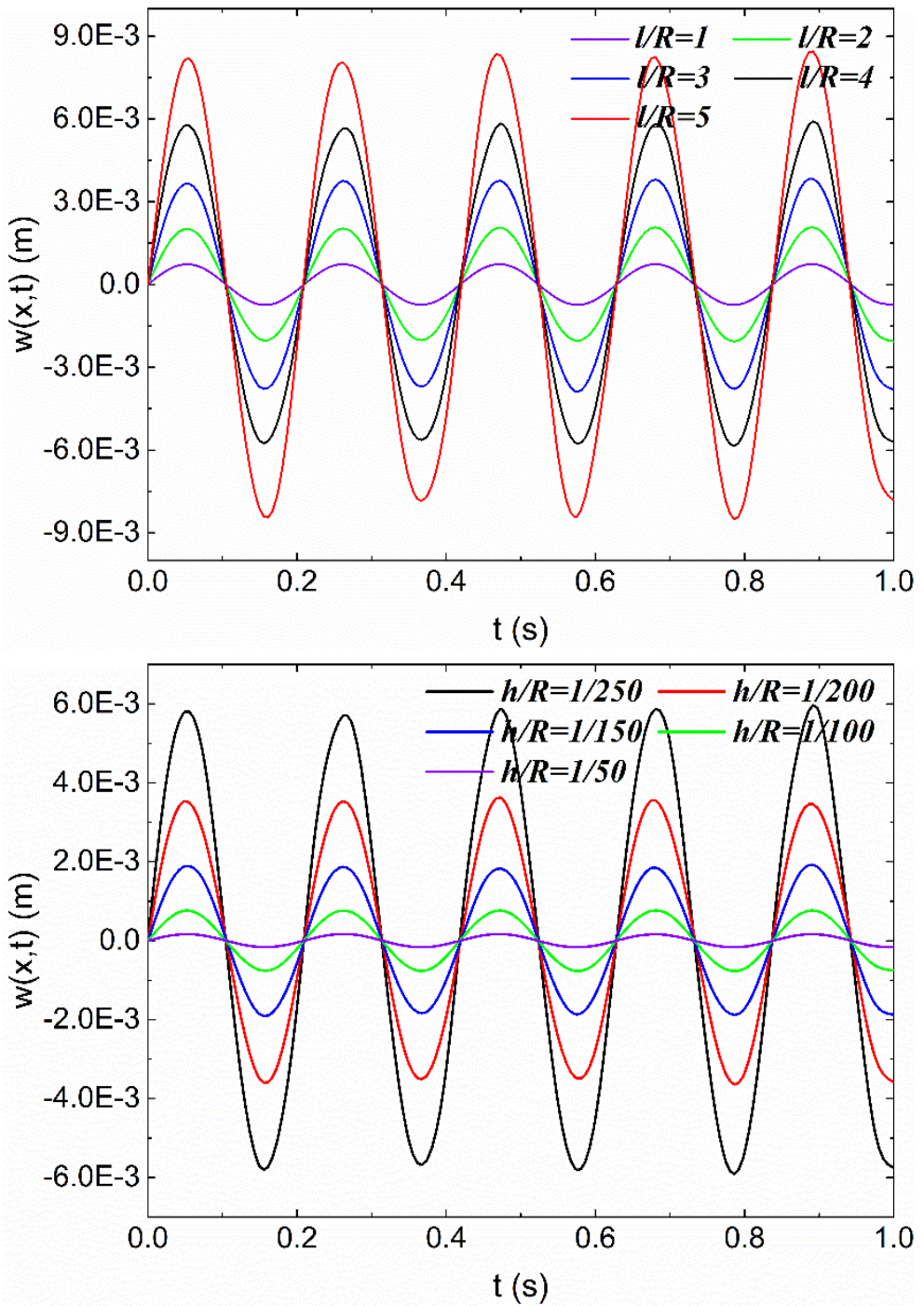

Fig. 7. The time-history responses of a cylindrical shell at a chosen position $(x=l / 2, \theta=0, z=0)$ under different length-radius ratios and thickness-radius ratios. 


\title{
A semi-analytical method for the dynamic analysis of cylindrical shells with arbitrary boundaries
}

Xu Liang ${ }^{1}$, Xing Zha ${ }^{1}$, Xue Jiang ${ }^{2, *}$, Zeng Cao ${ }^{1}$, Yuhong Wang ${ }^{1}$, Jianxing Leng ${ }^{1}$

${ }^{1}$ Ocean College, Zhejiang University, Hangzhou, Zhejiang 310058, China

${ }^{2}$ Dept. of Naval Architecture, Ocean and Marine Engineering, University of Strathclyde, Henry Dyer Building, Glasgow, G1 5AE, UK

* Corresponding Author: xue.jiang@ @strath.ac.uk

\begin{abstract}
The dynamic behavior of cylindrical shells with arbitrary boundaries is studied in this paper. Love's shell theory and Hamilton's principle are employed to derive the motion equations for cylindrical shells. A semi-analytical methodology, which incorporates Durbin's inverse Laplace transform, differential quadrature method and Fourier series expansion technique, is proposed to investigate this phenomenon. The use of the differential quadrature method provides a solution in terms of the axial direction whereas the use of Durbin's numerical inversion method generates a solution in the time domain. Comparison of calculated frequency parameters to that derived from the literature illustrates the effectiveness of the method. Specifically, convergence tests indicate that the present approach has a rapid convergence, the time-history response and the Navier's solution are in great agreement. Comparisons between time-history responses derived by two shell theories show that the results fit well with each other when the thickness-radius ratios are small enough. An analysis of the influences of boundaries on the time-history response of cylindrical shells indicates that the peak displacement is closely related to the degrees of freedom of boundaries. The influences of the length-radius ratios and the thickness-radius ratios on the peak displacement are further investigated.
\end{abstract}

Keywords: Time-history response; Frequency parameter; Differential quadrature method; Durbin's inverse Laplace transform.

\section{Introduction}

As an important structural component, the cylindrical shells have been widely used in ship construction and marine engineering, such as autonomous underwater vehicles, deep-ocean submersibles, subsea pipelines and nuclear facilities for their transportation ability, convenient storage and good load capacity (Gupta et al., 2016; Wu et al., 2018; Zhang et al., 2015a). These structures usually suffer from extreme loads including ocean currents, waves, and wind etc., resulting in vibration and damage of the structures (Zhang et al., 2015b). It is, therefore, necessary to exactly predict the vibration behavior of the cylindrical shells, as this knowledge can provide a theoretical foundation and scientific basis for structural design and performance optimization.

The extensive application of these structural elements has spurred rapid developments in shell theories and calculation methods for more accurate and efficient dynamic analysis of 
the cylindrical shells. The existing shell theories can be divided into three categories: the classical shell theory (CST), the first-order shear deformation theory (FSDT) and higher-order shear deformation theory (HSDT) (Thai and Kim, 2015). It is noted that CST is effective only in calculating the natural frequency and time-history response of thin cylindrical shells, FSDT and HSDT are more accurate in analyzing the dynamic behaviors of cylindrical shells. More details about the development of this research can be found in Leissa (1993), Qatu (2004) and Reddy (2003). Although the FSDT and HSDT are more accurate and precise, it is effective to choose CST to simulate the thin cylindrical shells under the consideration of computational loads and accuracy requirements.

Apart from the various shell theories, a variety of accurate and efficient calculation methods have been proposed one after another for vibration analysis of cylindrical shells, such as Rayleigh-Ritz method (Pradhan et al., 2000), Galerkin method (Haddadpour et al., 2007), discrete singular convolution method (Civalek, 2006, 2013), wave propagation approach (Liu et al., 2018), the transfer matrix method (Liang and Chen, 2006), finite element method (Kadoli and Ganesan, 2006; Santos et al., 2009), the generalized integral transform technique (Gu et al., 2013), meshless method (Ferreira et al., 2011; Ferreira et al., 2006), a domain decomposition approach ( $\mathrm{Qu}$ et al., 2013a; Qu et al., 2013b) and homotopy perturbation method (Yazdi, 2013) and so on. These methods mentioned above are proposed to derive the natural frequencies of shells and plates. However, the time-history response of cylindrical shells is rarely discussed in references. Reddy and Khdeir (1989) used the state space approach and the separation of variables technique to investigate the dynamic response of simply supported cross-ply laminated shallow shells under various loadings based on the third-order shear deformation shell theory. Lu and Lam (1995) combined the Rayleigh-Ritz method and the normal mode superposition method to evaluate the transient response of clamped laminated curved panels subjected to external loadings. Qing et al. (2008) presented the natural frequency, harmonic vibration and complex frequency response of simply supported laminated plates and shells with damping using the precise integration method and Muller method. Bodaghi and Shakeri (2012) developed an analytical approach to obtain the free vibration and dynamic response of the simply supported functionally graded piezo-electric cylindrical panel impacted by time-dependent blast pulses. Maleki et al. (2012) proposed a hybrid method to study the static and transient response of the moderately thick laminated cylindrical shell panels with various boundary conditions and loadings. Shao et al. (2017b) obtained the natural frequencies and transient response of cylindrical shells based on a simple first-order shear deformation shell theory using the method of reverberation ray matrix. Shao et al. (2017a) adopted an enhanced reverberation ray matrix approach to assess the transient response of composite laminated shallow shells with general boundary conditions based on the first-order shear deformation shallow shell theory and the classical shallow shell theory. Frikha et al. (2018) used a linear discrete double directors finite element model and Newmark's algorithm to study the dynamic behavior of functionally graded carbon nanotubes-reinforced shells. Duc (2013) incorporated the Bubnov-Galerkin method and Runge-Kutta method to evaluate the nonlinear transient response of imperfect shallow shells. Hajmohammad et al. (2017) chose the differential quadrature method and Newmark approach to investigate the dynamic response of submerged cylindrical shells under seismic action. Zhang et al. (2017) estimated the transient response of CVT-reinforced cylindrical shells 
subjected to impact loads by Fourier series expansion and Laplace transform.

Although the above methods can analyze the dynamic behavior of the cylindrical shells, it is difficult for them to take account of the calculation accuracy, efficiency and algorithm stability at the same time. Considering the limitations of these methods, a more precise and accurate method is needed to calculate the time-history response of cylindrical shells under arbitrary boundaries. In the present work, Love's shell theory and Hamilton's principle are employed to obtain the motion equations and a semi-analytical method that incorporates the differential quadrature method, Durbin's inverse Laplace transform is developed to predict the time-history response of cylindrical shells subjected to a harmonic load. This response is validated by comparing with the Navier's solution. The frequency parameters of cylindrical shells are then calculated and the influences of boundaries, length-radius ratios, and thickness-radius ratios on the time-history response of cylindrical shells are evaluated.

\section{Laplace transform and differential quadrature method}

\subsection{Laplace transform and its numerical inversion}

As an efficient and high precision computing method, the Laplace transform, which has been extensively used for solving partial differential equations, is expressed as follows:

$$
\tilde{h}(s)=\int_{0}^{+\infty} h(t) e^{-s t} d t
$$

in which $\sim$ denotes the transformed function and $s$ is a complex number.

Durbin developed a novel numerical inverse Laplace transform, which can give exact values of original functions in the interval $(0, T / 2)$, defined as (Durbin, 1974):

$$
h(t) \approx \frac{2 e^{\lambda t}}{T}\left\{\tilde{h}(\lambda) / 2+\sum_{k=1}^{K} \operatorname{Re}[\tilde{h}(\lambda+k \pi i / T)] \cos (k \pi t / T)\right\}
$$

in which $\lambda=5 / T, i$ is the imaginary unit in complex number and $K$ denotes a large integer.

\subsection{Differential quadrature method}

The principle of differential quadrature method is that the weighted sum of functional values of all grid points is used to represent a function and its derivatives at a chosen point, and it is defined as below (Liang et al., 2014):

$$
\frac{\partial^{i} g\left(x_{m}\right)}{\partial x^{i}}=\sum_{n=1}^{N} C_{m n}^{(i)} g\left(x_{n}\right) \quad(m, n=1,2, . . N)
$$

in which $N$ denotes the number of grid points, and $C_{\mathrm{mn}}^{(i)}$ refer to the weighting coefficients defined by:

$$
\begin{gathered}
C_{m n}^{(1)}=\frac{\prod_{r=1, r \neq n}^{N}\left(x_{m}-x_{r}\right)}{\left(x_{m}-x_{n}\right) \prod_{r=1, r \neq n}^{N}\left(x_{n}-x_{r}\right)} \\
C_{m n}^{(i)}=i\left(C_{m n}^{(i-1)} C_{m n}^{1}-\frac{C_{m n}^{(i-1)}}{x_{m}-x_{n}}\right)(i=2,3, \ldots N-1)
\end{gathered}
$$

in which $n \neq m$, and the $C_{\mathrm{mm}}^{(\mathrm{i})}$ are given as: 


$$
C_{m m}^{(i)}=-\sum_{n=1, n \neq m}^{N} C_{m n}^{(i)}(m=1,2 \ldots N, i=1,2 \ldots N-1)
$$

\section{Problem description}

\subsection{Governing equations}

A thin cylindrical shell as shown in Fig. 1 is considered, the symbols $l, R, h$ denote the length, radius, and thickness of the cylindrical shell. The origin of the cylindrical coordinate system is fixed at the mid-surface of the cylindrical shells.

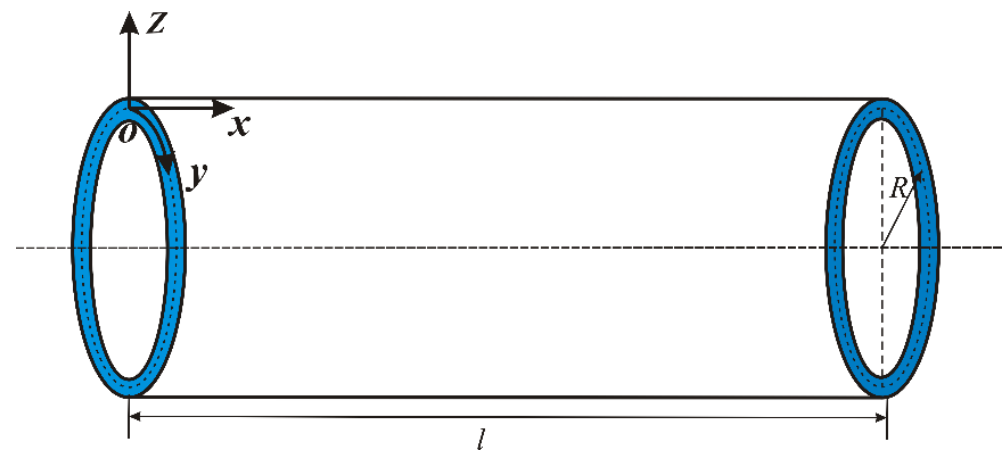

Fig. 1. Geometry of the thin cylindrical shell subjected to a harmonic load.

For thin shells, the following assumptions are considered: the shear deformation and rotary inertia are ignored, and the normal line of the mid-surface remains straight and normals to the mid-surface during deformation. The displacement-strain relationships based on Love's shell theory are expressed as:

$$
\boldsymbol{\varepsilon}=\left\{\begin{array}{c}
\varepsilon_{x} \\
\varepsilon_{\theta} \\
\gamma_{x \theta}
\end{array}\right\}=\boldsymbol{\varepsilon}_{0}+z \boldsymbol{\kappa}=\left\{\begin{array}{c}
\frac{\partial u_{0}}{\partial x} \\
\frac{1}{R}\left(\frac{\partial v_{0}}{\partial \theta}+w_{0}\right) \\
\frac{\partial v_{0}}{\partial x}+\frac{1}{R} \frac{\partial u_{0}}{\partial \theta}
\end{array}\right\}+z\left\{\begin{array}{c}
-\frac{\partial^{2} w_{0}}{\partial x^{2}} \\
\frac{1}{R^{2}}\left(\frac{\partial v_{0}}{\partial \theta}-\frac{\partial^{2} w_{0}}{\partial \theta^{2}}\right) \\
\frac{2}{R}\left(\frac{\partial v_{0}}{\partial x}-\frac{\partial^{2} w_{0}}{\partial x \partial \theta}\right)
\end{array}\right\}
$$

in which $\boldsymbol{\varepsilon}_{\mathbf{0}}$ is the strain vector and $\boldsymbol{\kappa}$ refers to the vector of curvature changes, $u_{0}, v_{0}, w_{0}$ denote displacement components on the mid-surface in $x$-, $\theta$ - and $z$-directions, respectively;

The stress-strain relationships are given as:

$$
\boldsymbol{\sigma}=\mathbf{Q} \boldsymbol{\varepsilon}
$$

in which $\boldsymbol{\sigma}$ is the stress vector, and $\mathbf{Q}$ is the elastic constants matrix defined as:

$$
\boldsymbol{\sigma}=\left\{\sigma_{x}, \sigma_{\theta}, \tau_{x \theta}\right\}, \mathbf{Q}=\left[\begin{array}{ccc}
Q_{11} & Q_{12} & 0 \\
Q_{12} & Q_{22} & 0 \\
0 & 0 & Q_{66}
\end{array}\right]
$$

For isotropic materials, the elastic constants $Q_{i j}(i, j=1,2$ and 6$)$ can be stated as:

$$
Q_{11}=Q_{22}=\frac{E}{1-\mu^{2}}, Q_{12}=\frac{\mu E}{1-\mu^{2}}, Q_{66}=\frac{E}{2(1+\mu)} .
$$

in which $E$ is Young's modulus, and $\mu$ is Poisson's ratio. 
The Hamilton's principle is employed to obtain motion equations for thin cylindrical shells, and it is defined as:

$$
\delta J=\int_{0}^{t}\left(\delta K-\delta V+\delta W_{n c}\right) d t=0
$$

in which $K$, and $V$ refer to the kinetic energy and strain energy, $W_{n c}$ denotes the work done by external loads.

The kinetic energy without considering the rotary inertia terms can be written as follows:

$$
K=\frac{1}{2} \int_{0}^{l} \int_{0}^{2 \pi} \int_{-h / 2}^{h / 2} \rho\left(\dot{u}_{0}^{2}+\dot{v}_{0}^{2}+\dot{w}_{0}^{2}\right) R d x d \theta d z
$$

in which the superscript "“" represents the variables with respect to time.

The strain energy is expressed as:

$$
V=\frac{1}{2} \int_{0}^{l} \int_{0}^{2 \pi} \int_{-h / 2}^{h / 2}\left(\sigma_{x} \varepsilon_{x}+\sigma_{\theta} \varepsilon_{\theta}+\tau_{x \theta} \gamma_{x \theta}\right) R d x d \theta d z
$$

The work done by external loads is defined by:

$$
\delta W_{n c}=\int_{0}^{l} \int_{0}^{2 \pi}\left(f_{x} \delta u_{0}+f_{\theta} \delta v_{0}+f_{z} \delta w_{0}\right) R d x d \theta
$$

in which $f_{x}, f_{\theta}$ and $f_{z}$ stand for external loads in $x$-, $\theta$ - and $z$ - directions, respectively, and it is noteworthy that $f_{x}=0, f_{\theta}=0, f_{z}=3000 \cos (300 \mathrm{t}) \delta(\theta)$.

Substituting Eqs. (12)-(14) into Eq. (11), the motion equations for thin cylindrical shells in terms of stress resultants are given as:

$$
\begin{gathered}
\frac{\partial N_{x}}{\partial x}+\frac{1}{R} \frac{\partial N_{x \theta}}{\partial \theta}+f_{x}=I_{0} \frac{\partial^{2} u_{0}}{\partial t^{2}} \\
\frac{\partial N_{x \theta}}{\partial x}+\frac{1}{R} \frac{\partial N_{\theta}}{\partial \theta}+\frac{1}{R} \frac{\partial M_{x \theta}}{\partial x}+\frac{1}{R^{2}} \frac{\partial M_{\theta}}{\partial \theta}+f_{\theta}=I_{0} \frac{\partial^{2} v_{0}}{\partial t^{2}} \\
\frac{\partial^{2} M_{x}}{\partial x^{2}}+\frac{2}{R} \frac{\partial^{2} M_{x \theta}}{\partial x \partial \theta}+\frac{1}{R^{2}} \frac{\partial^{2} M_{\theta}}{\partial \theta^{2}}-\frac{N_{\theta}}{R}+f_{z}=I_{0} \frac{\partial^{2} w_{0}}{\partial t^{2}}
\end{gathered}
$$

in which

$$
\begin{aligned}
\left\{N_{x}, N_{\theta}, N_{x \theta}\right\} & =\int_{-h / 2}^{h / 2}\left\{\sigma_{x}, \sigma_{\theta}, \tau_{x \theta}\right\} d z \\
\left\{M_{x}, M_{\theta}, M_{x \theta}\right\} & =\int_{-h / 2}^{h / 2} z\left\{\sigma_{x}, \sigma_{\theta}, \tau_{x \theta}\right\} d z \\
I_{0} & =\int_{-h / 2}^{h / 2} \rho d z
\end{aligned}
$$

By substituting Eqs. (7)-(10) into Eq. (15), the motion equations in terms of the displacement components $\left(u_{0}, v_{0}, w_{0}\right)$ can be written as: 
in which

$$
\left\{A_{i j}, B_{i j}, D_{i j}\right\}=\int_{-h / 2}^{h / 2}\left\{1, z, z^{2}\right\} Q_{i j} d z, \quad(i, j=1,2,6) .
$$

\subsection{The boundary conditions at the edges}

The boundaries of the elastically restrained shell are described by setting the values of translational springs and rotational spring, and the boundaries of cylindrical shells can be stated as follows (Jin et al., 2015; Jin et al., 2013):

At left edge $(x=0)$ :

$$
\begin{gathered}
k_{u 0} u_{0}=N_{x}, \quad k_{v 0} v_{0}=N_{x \theta}+\frac{M_{x \theta}}{R}, \\
k_{w 0} w_{0}=Q_{x}+\frac{\partial M_{x \theta}}{R \partial \theta}, K_{w 0} \frac{\partial w_{0}}{\partial x}=-M_{x} .
\end{gathered}
$$

At right edge $(x=l)$ :

$$
\begin{gathered}
k_{u L} u_{0}=-N_{x}, \quad k_{v L} v_{0}=-N_{x \theta}-\frac{M_{x \theta}}{R}, \\
k_{w L} w_{0}=-Q_{x}-\frac{\partial M_{x \theta}}{R \partial \theta}, \quad K_{w L} \frac{\partial w_{0}}{\partial x}=M_{x} .
\end{gathered}
$$

in which $k_{u}, k_{v}$ and $k_{w}$ denote the translational spring stiffness coefficients, $K_{w}$ is the rotational spring stiffness.

The stiffness coefficients of springs corresponding to four boundaries are defined as:

Free edge $(\mathrm{F})$ :

$$
k_{u}=k_{v}=k_{w}=K_{w}=0
$$

Simply-supported edge (S):

$$
k_{u}=k_{v}=k_{w}=10^{14}, K_{w}=0 .
$$


Shear-diaphragm edge (SD):

$$
k_{u}=K_{w}=0, k_{v}=k_{w}=10^{14} .
$$

Clamped edge (C):

$$
k_{u}=k_{v}=k_{w}=K_{w}=10^{14} .
$$

\section{Solution process}

\subsection{Natural frequency solution}

The modal frequencies of cylindrical shells are calculated, and the displacement components of cylindrical shells for modal analysis are expanded as:

$$
\left\{\begin{array}{c}
u_{0}(x, \theta, t)=U(x) \cos (k \theta) e^{i \omega t}, \\
v_{0}(x, \theta, t)=V(x) \sin (k \theta) e^{i \omega t}, \\
w_{0}(x, \theta, t)=W(x) \cos (k \theta) e^{i \omega t} .
\end{array}\right.
$$

in which $k$ and $\omega$ denote the circumferential wavenumber and natural frequency of the cylindrical shell, respectively.

By substituting Eq. (27) into the motion equations without considering external forces, and then employing the DQM on the resulting equations, the following formulas can be obtained:

$$
\begin{gathered}
-\frac{A_{66} k^{2}}{R} U_{i}+A_{11} R \sum_{j=1}^{N} A_{i j}^{(2)} U_{j}+\left(A_{12} k+A_{66} k+\frac{B_{12} k}{R}+\frac{2 B_{66} k}{R}\right) \sum_{j=1}^{N} A_{i j}^{(1)} V_{j} \\
+\left(A_{12}+\frac{B_{12} k^{2}}{R}+\frac{2 B_{66} k^{2}}{R}\right) \sum_{j=1}^{N} A_{i j}^{(1)} W_{j}-B_{11} R \sum_{j=1}^{N} A_{i j}^{(3)} W_{j}+\omega^{2} I_{0} R U_{i}=0, \\
\left(-A_{12} k-A_{66} k-\frac{B_{12} k}{R}-\frac{2 B_{66} k}{R}\right) \sum_{j=1}^{N} A_{i j}^{(1)} U_{j}+\left(-\frac{D_{22} k^{2}}{R^{3}}-\frac{2 B_{22} k^{2}}{R^{2}}-\frac{A_{22} k^{2}}{R}\right) V_{i} \\
+\left(4 B_{66}+\frac{4 D_{66}}{R}+A_{66} R\right) \sum_{j=1}^{N} A_{i j}^{(2)} V_{j}+\left(-\frac{D_{22} k^{3}}{R^{3}}-\frac{B_{22} k}{R^{2}}-\frac{B_{22} k^{3}}{R^{2}}-\frac{A_{22} k}{R}\right) W_{i} \\
+\left(B_{12} k+2 B_{66} k+\frac{D_{12} k}{R}+\frac{4 D_{66} k}{R}\right) \sum_{j=1}^{N} A_{i j}^{(2)} W_{j}+\omega^{2} I_{0} R V_{i}=0, \\
\left.+-A_{12}-\frac{B_{12} k^{2}}{R}-\frac{2 B_{66} k^{2}}{R}\right) \sum_{j=1}^{N} A_{i j}^{(1)} U_{j}+B_{11} R \sum_{j=1}^{N} A_{i j}^{(3)} U_{j}+\left(-\frac{D_{22} k^{3}}{R^{3}}-\frac{B_{22} k}{R^{2}}-\frac{B_{22} k^{3}}{R^{2}}-\frac{A_{22} k}{R}\right) V_{i} \\
+\left(B_{12} k+2 B_{66} k+\frac{D_{12} k}{R}+\frac{4 D_{66} k}{R}\right) \sum_{j=1}^{N} A_{i j}^{(2)} V_{j}+\left(-\frac{D_{22} k^{4}}{R^{3}}-\frac{2 B_{22} k^{2}}{R^{2}}-\frac{A_{22}}{R}\right) W_{i} \\
+\left(2 B_{12}+\frac{2 D_{12} k^{2}}{R}+\frac{4 D_{66} k^{2}}{R}\right) \sum_{j=1}^{N} A_{i j}^{(2)} W_{j}-D_{11} R \sum_{j=1}^{N} A_{i j}^{(4)} W_{j}+\omega^{2} I_{0} R W_{i}=0 .
\end{gathered}
$$

Substituting boundaries into Eq. (28), the obtained equations are rewritten into matrix form as: 


$$
\begin{gathered}
{\left[\begin{array}{ll}
\mathbf{k}_{\mathbf{b b}} & \mathbf{k}_{\mathbf{b d}} \\
\mathbf{k}_{\mathbf{d b}} & \mathbf{k}_{\mathbf{d d}}
\end{array}\right]\left\{\begin{array}{l}
\mathbf{u}_{\mathbf{b}} \\
\mathbf{u}_{\mathbf{d}}
\end{array}\right\}+\omega^{2}\left[\begin{array}{ll}
\mathbf{s}_{\mathbf{b b}} & \mathbf{s}_{\mathbf{b d}} \\
\mathbf{s}_{\mathbf{d b}} & \mathbf{s}_{\mathbf{d d}}
\end{array}\right]\left\{\begin{array}{l}
\mathbf{u}_{\mathbf{b}} \\
\mathbf{u}_{\mathbf{d}}
\end{array}\right\}=\left\{\begin{array}{l}
\mathbf{0} \\
\mathbf{0}
\end{array}\right\}} \\
\mathbf{u}_{\mathbf{b}}^{\mathbf{T}}=\left\{U_{1}, U_{n}, V_{1}, V_{n}, W_{1}, W_{2}, W_{n-1}, W_{n}\right\}, \\
\mathbf{u}_{\mathbf{d}}^{\mathbf{T}}=\left\{U_{2}, \ldots, U_{n-1}, V_{2}, \ldots, V_{n-1}, W_{3}, \ldots, W_{n-2}\right\} .
\end{gathered}
$$

After eliminating the vector $\boldsymbol{u}_{\boldsymbol{b}}$, the resulting equations can be described as:

$$
\left(\omega^{2} \mathbf{M}+\mathbf{K}\right) \mathbf{u}_{\mathbf{d}}=\mathbf{0}
$$

in which $\boldsymbol{M}$ denotes the mass matrix, $\boldsymbol{K}$ is stiffness matrix, and $\boldsymbol{u}_{\boldsymbol{d}}$ refer to a vector. The eigenvalues of the following matrix are the natural frequencies, and the eigenvalue matrix is given as:

$$
\mathbf{E}=\left[\begin{array}{cc}
\mathbf{0} & \mathbf{I} \\
-\mathbf{M}^{-1} \mathbf{K} & \mathbf{0}
\end{array}\right]
$$

\subsection{Transient response solution}

The trigonometric series are taken to expand the displacement components and external loads on the mid-surface of the cylindrical shells in the circumferential direction, and the detailed expansion forms of displacement components and external loads are presented as:

$$
\begin{array}{ll}
\tilde{u}_{0}=\sum_{k=1}^{\infty} \tilde{\overline{\bar{u}}}_{0}(x, s) \cos k \theta, & \tilde{f}_{x}=\sum_{k=1}^{\infty} \tilde{\overline{\bar{f}}}(x, s) \cos k \theta, \\
\tilde{v}_{0}=\sum_{k=1}^{\infty} \tilde{\bar{v}}_{0}(x, s) \sin k \theta, & \tilde{f}_{\theta}=\sum_{k=1}^{\infty} \tilde{\bar{f}}_{\theta}(x, s) \sin k \theta, \\
\tilde{w}_{0}=\sum_{k=1}^{\infty} \tilde{\bar{w}}_{0}(x, s) \cos k \theta, & \tilde{f}_{z}=\sum_{k=1}^{\infty} \tilde{\bar{f}}_{z}(x, s) \cos k \theta .
\end{array}
$$

Substituting Eq. (32) into Eq. (19) and the motion equations processed by Laplace transform are expressed as: 


$$
\begin{gathered}
\left(-\frac{A_{66} k^{2}}{R}-I_{0} R s^{2}\right) \tilde{\bar{u}}_{0}+A_{11} R \frac{\partial^{2} \tilde{\bar{u}}_{0}}{\partial x^{2}}+\left(A_{12} k+A_{66} k+\frac{B_{12} k}{R}+\frac{2 B_{66} k}{R}\right) \frac{\partial \tilde{\bar{v}}_{0}}{\partial x} \\
+\left(A_{12}+\frac{B_{12} k^{2}}{R}+\frac{2 B_{66} k^{2}}{R}\right) \frac{\partial \tilde{\bar{w}}_{0}}{\partial x}-B_{11} R \frac{\partial^{3} \tilde{\bar{w}}_{0}}{\partial x^{3}}+\tilde{\bar{f}}_{x} R=0, \\
\left(-A_{12} k-A_{66} k-\frac{B_{12} k}{R}-\frac{2 B_{66} k}{R}\right) \frac{\partial \tilde{\bar{u}}_{0}}{\partial x}+\left(4 B_{66}+\frac{4 D_{66}}{R}+A_{66} R\right) \frac{\partial^{2} \tilde{\bar{v}}_{0}}{\partial x^{2}} \\
+\left(-\frac{D_{22} k^{2}}{R^{3}}-\frac{2 B_{22} k^{2}}{R^{2}}-\frac{A_{22} k^{2}}{R}-I_{0} R s^{2}\right) \tilde{\bar{v}}_{0}+\left(-\frac{D_{22} k^{3}}{R^{3}}-\frac{B_{22} k}{R^{2}}-\frac{B_{22} k^{3}}{R^{2}}-\frac{A_{22} k}{R}\right) \tilde{\bar{w}}_{0} \\
+\left(B_{12} k+2 B_{66} k+\frac{D_{12} k}{R}+\frac{4 D_{66} k}{R}\right) \frac{\partial^{2} \tilde{\bar{w}}_{0}}{\partial x^{2}}+\tilde{\bar{f}}_{\theta} R=0, \\
\left(-A_{12}-\frac{B_{12} k^{2}}{R}-\frac{2 B_{66} k^{2}}{R}\right) \frac{\partial \tilde{\bar{u}}_{0}}{\partial x}+B_{11} R \frac{\partial^{3} \tilde{\bar{u}}_{0}}{\partial x^{3}}+\left(-\frac{D_{22} k^{3}}{R^{3}}-\frac{B_{22} k}{R^{2}}-\frac{B_{22} k^{3}}{R^{2}}-\frac{A_{22} k}{R}\right) \tilde{\bar{v}}_{0}+ \\
\left(B_{12} k+2 B_{66} k+\frac{D_{12} k}{R}+\frac{4 D_{66} k}{R}\right) \frac{\partial^{2} \tilde{\bar{v}}_{0}}{\partial x^{2}}+\left(-\frac{D_{22} k^{4}}{R^{3}}-\frac{2 B_{22} k^{2}}{R^{2}}-\frac{A_{22}}{R}-I_{0} R s^{2}\right) \tilde{\bar{w}}_{0} \\
+\left(2 B_{12}+\frac{2 D_{12} k^{2}}{R}+\frac{4 D_{66} k^{2}}{R}\right) \frac{\partial^{2} \tilde{\bar{w}}_{0}}{\partial x^{2}}-D_{11} R \frac{\partial^{4} \tilde{\bar{w}}_{0}}{\partial x^{4}}+\tilde{\bar{f}}_{z} R=0 .
\end{gathered}
$$

Employing the DQM on Eq. (33) and the following equations can be given:

$$
\begin{gathered}
\left(-\frac{A_{66} k^{2}}{R}-I_{0} R s^{2}\right) \tilde{\bar{u}}_{0 i}+A_{11} R \sum_{j=1}^{N} A_{i j}^{(2)} \tilde{\bar{u}}_{0 j}+\left(A_{12} k+A_{66} k+\frac{B_{12} k}{R}+\frac{2 B_{66} k}{R}\right) \sum_{j=1}^{N} A_{i j}^{(1)} \tilde{\bar{v}}_{0 j} \\
+\left(A_{12}+\frac{B_{12} k^{2}}{R}+\frac{2 B_{66} k^{2}}{R}\right) \sum_{j=1}^{N} A_{i j}^{(1)} \tilde{\bar{w}}_{0 j}-B_{11} R \sum_{j=1}^{N} A_{i j}^{(3)} \tilde{\bar{w}}_{0 j}+\tilde{\bar{f}}_{x i} R=0, \\
\left(-A_{12} k-A_{66} k-\frac{B_{12} k}{R}-\frac{2 B_{66} k}{R}\right) \sum_{j=1}^{N} A_{i j}^{(1)} \tilde{\bar{u}}_{0 j}+\left(4 B_{66}+\frac{4 D_{66}}{R}+A_{66} R\right) \sum_{j=1}^{N} A_{i j}^{(2)} \tilde{\bar{v}}_{0 j} \\
+\left(-\frac{D_{22} k^{2}}{R^{3}}-\frac{2 B_{22} k^{2}}{R^{2}}-\frac{A_{22} k^{2}}{R}-I_{0} R s^{2}\right) \tilde{\bar{v}}_{0 i}+\left(-\frac{D_{22} k^{3}}{R^{3}}-\frac{B_{22} k}{R^{2}}-\frac{B_{22} k^{3}}{R^{2}}-\frac{A_{22} k}{R}\right) \tilde{\bar{w}}_{0 i} \\
+\left(B_{12} k+2 B_{66} k+\frac{D_{12} k}{R}+\frac{4 D_{66} k}{R}\right) \sum_{j=1}^{N} A_{i j}^{(2)} \tilde{\bar{w}}_{0 j}+\tilde{\bar{f}}_{\theta i} R=0, \\
\left(-A_{12}-\frac{B_{12} k^{2}}{R}-\frac{2 B_{66} k^{2}}{R}\right) \sum_{j=1}^{N} A_{i j}^{(1)} \tilde{\bar{u}}_{0 j}+B_{11} R \sum_{j=1}^{N} A_{i j}^{(3)} \tilde{\bar{u}}_{0 j}+\left(-\frac{D_{22} k^{3}}{R^{3}}-\frac{B_{22} k}{R^{2}}-\frac{B_{22} k^{3}}{R^{2}}-\frac{A_{22} k}{R}\right) \tilde{\bar{v}}_{0 i}+ \\
\left(B_{12} k+2 B_{66} k+\frac{D_{12} k}{R}+\frac{4 D_{66} k}{R}\right) \sum_{j=1}^{N} A_{i j}^{(2)} \tilde{\bar{v}}_{0 j}+\left(-\frac{D_{22} k^{4}}{R^{3}}-\frac{2 B_{22} k^{2}}{R^{2}}-\frac{A_{22}}{R}-I_{0} R s^{2}\right) \tilde{\bar{w}}_{0 i} \\
+\left(2 B_{12}+\frac{2 D_{12} k^{2}}{R}+\frac{4 D_{66} k^{2}}{R}\right) \sum_{j=1}^{N} A_{i j}^{(2)} \tilde{\bar{w}}_{0 j}-D_{11} R \sum_{j=1}^{N} A_{i j}^{(4)} \tilde{\bar{w}}_{0 j}+\tilde{\bar{f}}_{z i} R=0 .
\end{gathered}
$$

The matrix form of Eq. (34) can be stated as:

$$
\mathbf{H} \cdot \mathbf{U}=\mathbf{F}
$$

in which 


$$
\begin{gathered}
\mathbf{U}=\left\{\begin{array}{c}
\mathbf{u}_{\mathbf{0}} \\
\mathbf{v}_{\mathbf{0}} \\
\mathbf{w}_{\mathbf{0}}
\end{array}\right\}, \mathbf{F}=\left\{\begin{array}{c}
\mathbf{f}_{\mathbf{x}} \\
\mathbf{f}_{\boldsymbol{\theta}} \\
\mathbf{f}_{\mathbf{z}}
\end{array}\right\}, \mathbf{H}=\left\{\begin{array}{lll}
\mathbf{H}_{\mathbf{1 1}} & \mathbf{H}_{\mathbf{1 2}} & \mathbf{H}_{\mathbf{1 3}} \\
\mathbf{H}_{\mathbf{2 1}} & \mathbf{H}_{\mathbf{2 2}} & \mathbf{H}_{\mathbf{2 3}} \\
\mathbf{H}_{\mathbf{3 1}} & \mathbf{H}_{\mathbf{3 2}} & \mathbf{H}_{\mathbf{3 3}}
\end{array}\right\}, \\
\mathbf{u}_{\mathbf{0}}^{T}=\left\{\tilde{\overline{\bar{u}}}_{01}, \ldots \tilde{\overline{\bar{u}}}_{0 j}, \ldots \tilde{\overline{\bar{u}}}_{0 N}\right\}, \mathbf{v}_{\mathbf{0}}^{T}=\left\{\tilde{\overline{\bar{v}}}_{01}, \ldots \tilde{\overline{\bar{v}}}_{0 j}, \ldots \tilde{\overline{\bar{v}}}_{0 N}\right\}, \mathbf{w}_{\mathbf{0}}^{T}=\left\{\tilde{\overline{\bar{w}}}_{01}, \ldots \tilde{\overline{\bar{w}}}_{0 j}, \ldots \tilde{\overline{\bar{w}}}_{0 N}\right\}, \\
\mathbf{f}_{\mathbf{x}}^{T}=-R\left\{\tilde{\overline{\bar{f}}}_{x 1}, \ldots \tilde{\bar{f}}_{x j}, \ldots \tilde{\bar{f}}_{x N}\right\}, \mathbf{f}_{\boldsymbol{\theta}}^{T}=-R\left\{\tilde{\overline{\bar{f}}}_{\theta 1}, \ldots \tilde{\bar{f}}_{\theta j}, \ldots \tilde{\bar{f}}_{\theta N}\right\}, \mathbf{f}_{\mathbf{z}}^{T}=-R\left\{\tilde{\bar{f}}_{z 1}, \ldots \tilde{\bar{f}}_{z j}, \ldots \tilde{\bar{f}}_{z N}\right\} .
\end{gathered}
$$

The expressions of matrix $\mathbf{H}$ are detailed in Appendix A.

The general boundaries of the elastically restrained shells processed by the trigonometric series expansion and Laplace transform can be stated as $(x=0)$ :

$$
\begin{gathered}
T_{11} \frac{\partial \tilde{\bar{u}}_{0}}{\partial x}+T_{12} \tilde{\bar{v}}_{0}+T_{13} \tilde{\bar{w}}_{0}=k_{u 0} \tilde{\bar{u}}_{0}, \\
T_{21} \tilde{\bar{u}}_{0}+T_{22} \frac{\partial \tilde{\bar{v}}_{0}}{\partial x}+T_{23} \frac{\partial \tilde{\bar{w}}_{0}}{\partial x}=k_{v 0} \tilde{\bar{v}}_{0}, \\
T_{32} \frac{\partial \tilde{\bar{v}}_{0}}{\partial x}+T_{331} \frac{\partial \tilde{\bar{w}}_{0}}{\partial x}+T_{332} \frac{\partial^{3} \tilde{\bar{w}}_{0}}{\partial x^{3}}=k_{w 0} \tilde{\bar{w}}_{0}, \\
T_{42} \tilde{\bar{v}}_{0}+T_{431} \tilde{\overline{\bar{w}}}_{0}+T_{432} \frac{\partial^{2} \tilde{\bar{w}}_{0}}{\partial x^{2}}=K_{w 0} \frac{\partial \tilde{\bar{w}}_{0}}{\partial x} .
\end{gathered}
$$

in which

$$
\begin{gathered}
T_{11}=\frac{E h}{1-\mu^{2}}, T_{12}=\frac{E h}{1-\mu^{2}} \frac{\mu k}{R}, T_{13}=\frac{E h}{1-\mu^{2}} \frac{\mu}{R}, T_{21}=-\frac{k}{R} \frac{E h}{2(1+\mu)}, \\
T_{22}=\frac{E h}{2(1+\mu)}\left(1+\frac{h^{2}}{6 R^{2}}\right), T_{23}=\frac{E h}{2(1+\mu)} \frac{h^{2} k}{6 R^{2}}, T_{32}=\frac{E h^{3} k}{12\left(1-\mu^{2}\right)}\left(\frac{\mu}{2 R^{2}}+\frac{1-\mu}{R^{2}}\right), \\
T_{331}=\frac{E h^{3} k^{2}}{12\left(1-\mu^{2}\right)}\left(\frac{\mu}{2 R^{2}}+\frac{1-\mu}{R^{2}}\right), T_{332}=-\frac{1}{2} \frac{E h^{3}}{12\left(1-\mu^{2}\right)}, \\
T_{42}=-\frac{\mu k}{R^{2}} \frac{E h^{3}}{12\left(1-\mu^{2}\right)}, T_{431}=-\frac{\mu k^{2}}{R^{2}} \frac{E h^{3}}{12\left(1-\mu^{2}\right)}, T_{432}=\frac{E h^{3}}{12\left(1-\mu^{2}\right)} .
\end{gathered}
$$

By substituting Eq. (37) into Eq. (35), and the elements corresponding to boundaries in matrices $\mathbf{H}_{\mathrm{ij}}$ are available in Appendix B.

After finding the solution of Eq. (35), the time-history responses of cylindrical shells are obtained by using the Durbin's inverse Laplace transform.

\section{Numerical results}

A Mathematica package was developed to present the numerical results in this paper, and the following steps are taken to evaluate the model. Firstly, the frequency parameters of thin cylindrical shells are calculated. Subsequently, the convergence of results for different numbers of grid points $(N)$ in the axial direction and the validity of the present method is estimated. Thirdly, the time-history responses derived by classical shell theory and first-order shear deformation theory are compared. Fourthly, the influences of various boundaries on cylindrical shell displacement are investigated. Lastly, the influences of length-radius ratios $(l / R)$ and thickness-radius ratios $(h / R)$ on time-history response are assessed. Considering a set 
of model parameters used in following numerical calculation, the system parameters used in the present work are given as follows: $E=70 \mathrm{GPa}, \mu=0.3, \rho=2707 \mathrm{~kg} / \mathrm{m}^{3}, l=2 \mathrm{~m}, R=0.5 \mathrm{~m}, h=0.002 \mathrm{~m}$.

In order to guarantee the convergence and accuracy of the results, the Chebyshev-Gauss-Lobatto grid points are used in present work and are defined as:

$$
x_{\kappa}=\frac{l}{2}\left(1-\cos \left(\frac{\kappa-1}{N-1}\right) \pi\right), \kappa=1,2, \ldots . N
$$

in which $N$ denotes the number of grid points in the axial direction, and it is taken as 11 in the following numerical examples.

\subsection{Natural frequencies}

To prove the correctness of the present method for the modal analysis of thin cylindrical shells, a series of calculated results will be compared with the frequency parameters from the literature. The length-radius ratio $l / R=20$ and Poisson's ratio $\mu=0.3$ are adopted in the following calculations. Table 1 presents the frequency parameters of cylindrical shells under three different boundaries, $m$ and $k$ refer to the axial and circumferential wavenumbers, respectively. It can be found from Table 1 that the frequency parameters produced by the differential quadrature method are consistent with the results obtained by Loy et al. (1997). Table 2 shows the frequency parameters of a SD-SD cylindrical shell under different thickness-radius ratios. As shown in Table 2, the frequency parameters generated by the proposed method fits well with the results calculated by Markuš (1988) and Loy et al. (1997). The frequency parameters of a different cylindrical shell with C-SD boundary are again compared in Table 3, which also shows a good agreement between the results produced by the present method and results from the literature.

Table 1 Frequency parameters of a thin cylindrical shell with three different boundaries $(h / R=0.01)$.

\begin{tabular}{ccccccc}
\hline & \multicolumn{5}{c}{ Frequency parameters $\Omega=\omega R \sqrt{\left(1-\mu^{2}\right) \rho / E}$} \\
\cline { 2 - 7 }$k$ & \multicolumn{2}{c}{ SD-SD } & \multicolumn{2}{c}{ C-C } & \multicolumn{2}{c}{ C-SD } \\
\cline { 2 - 7 } & $\begin{array}{c}\text { Loy et al. } \\
(1997)\end{array}$ & Present & $\begin{array}{c}\text { Loy et al. } \\
(1997)\end{array}$ & Present & $\begin{array}{c}\text { Loy et al. } \\
(1997)\end{array}$ & Present \\
\hline 1 & 0.016101 & 0.016101 & 0.032885 & 0.032884 & 0.023974 & 0.023976 \\
2 & 0.009382 & 0.009378 & 0.013923 & 0.013929 & 0.011225 & 0.011221 \\
3 & 0.022105 & 0.022103 & 0.022672 & 0.022668 & 0.022310 & 0.022308 \\
4 & 0.042095 & 0.042094 & 0.042208 & 0.042207 & 0.042139 & 0.042137 \\
5 & 0.068008 & 0.068007 & 0.068046 & 0.068045 & 0.068024 & 0.068023 \\
6 & 0.099730 & 0.099729 & 0.099748 & 0.099747 & 0.099738 & 0.099737 \\
7 & 0.137239 & 0.137238 & 0.137249 & 0.137249 & 0.137244 & 0.137243 \\
8 & 0.180527 & 0.180527 & 0.180535 & 0.180534 & 0.180531 & 0.180531 \\
9 & 0.229594 & 0.229593 & 0.229599 & 0.229598 & 0.229596 & 0.229596 \\
10 & 0.284435 & 0.284435 & 0.284439 & 0.284439 & 0.284437 & 0.284437 \\
\hline
\end{tabular}

Table 2 Frequency parameters of SD-SD cylindrical shells. 
Frequency parameters $\Omega=\omega R \sqrt{\left(1-\mu^{2}\right) \rho / E}$

\begin{tabular}{ccccccc}
\hline & \multicolumn{3}{c}{$h / R=0.05$} & & \multicolumn{3}{c}{$h / R=0.002$} \\
\cline { 2 - 7 }$k$ & $\begin{array}{c}\text { Markuš } \\
(1988)\end{array}$ & $\begin{array}{c}\text { Loy et al. } \\
(1997)\end{array}$ & Present & $\begin{array}{c}\text { Markuš } \\
(1988)\end{array}$ & $\begin{array}{c}\text { Loy et al. } \\
(1997)\end{array}$ & Present \\
\hline 1 & 0.0161063 & 0.01610 & 0.0161029 & 0.0161011 & 0.016101 & 0.0161011 \\
2 & 0.0392332 & 0.03930 & 0.0392710 & 0.00545243 & 0.005453 & 0.00545297 \\
3 & 0.109477 & 0.109824 & 0.1098116 & 0.00503724 & 0.005042 & 0.00504148 \\
4 & 0.209008 & 0.210284 & 0.2102773 & 0.00853409 & 0.008534 & 0.00853382 \\
\hline
\end{tabular}

Table 3 Frequency parameters of C-SD cylindrical shells $(h / R=0.002)$.

Frequency parameters $\Omega=\omega R \sqrt{\left(1-\mu^{2}\right) \rho / E}$

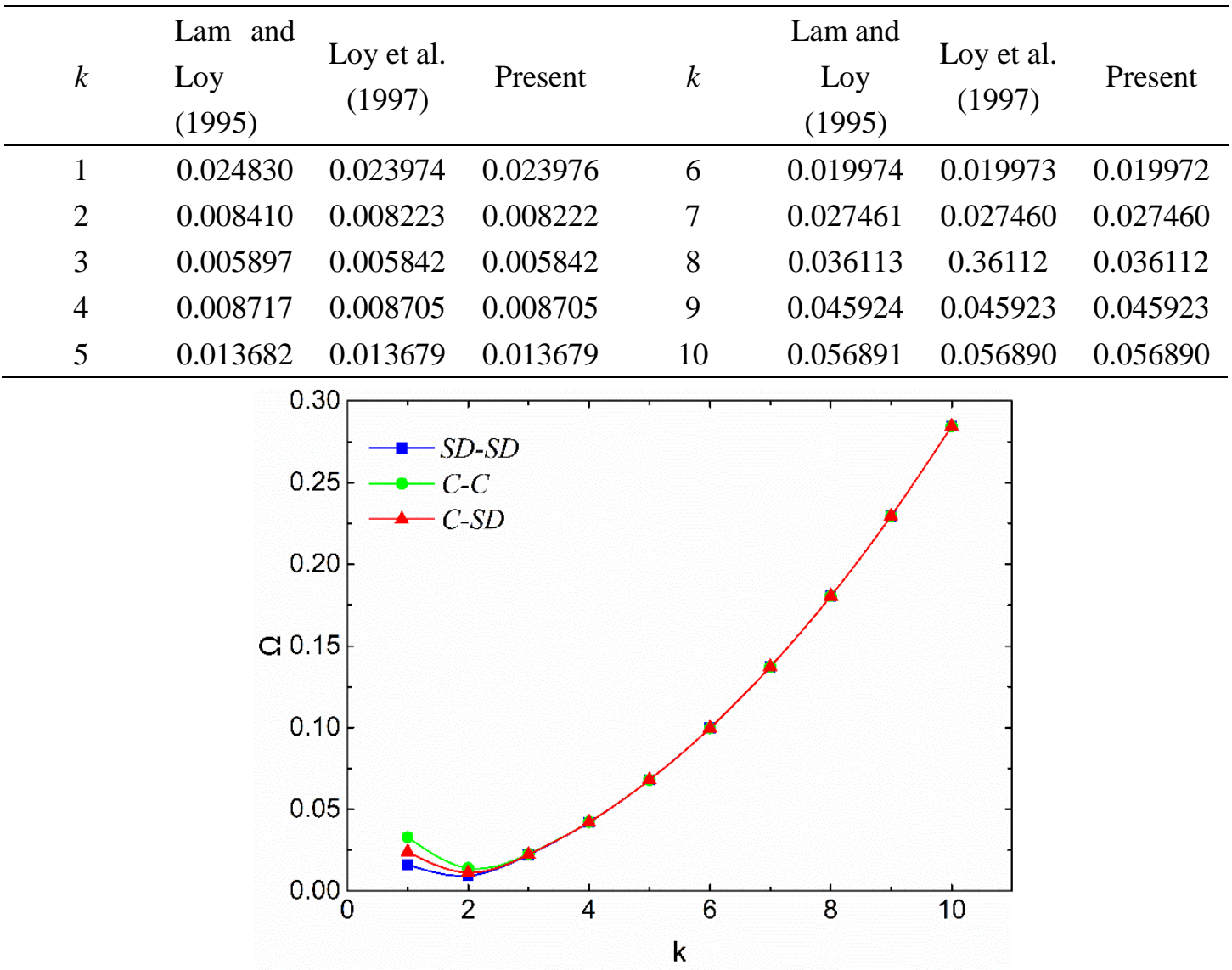

(a) different boundaries 


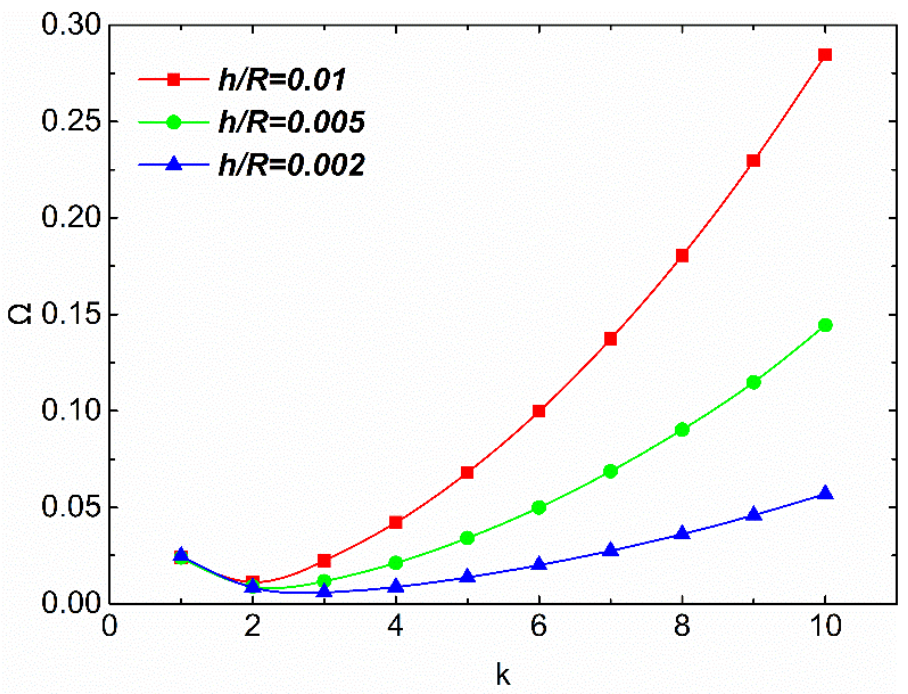

(b) different thickness-radius ratios $h / R$

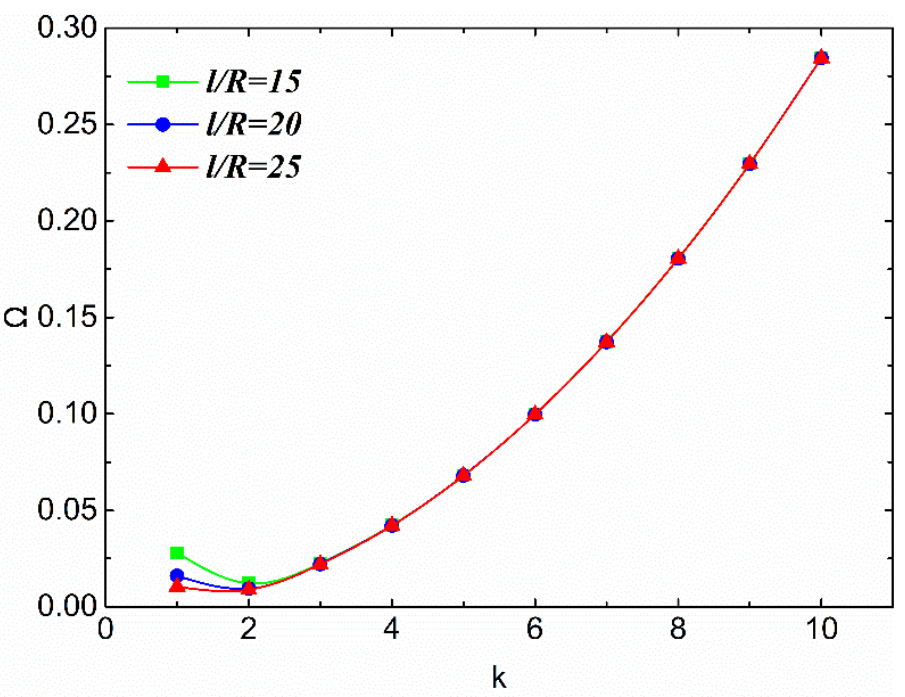

(c) different length-radius ratios $l / R$

Fig. 2. Variations of frequency parameters versus circumferential wavenumber $k$ : (a) different boundaries; (b) different thickness-radius ratios $h / R$.; (c) different length-radius ratios $l / R$.

Frequency parameters of a cylindrical shell with different boundaries, thickness-radius ratios, and length-radius ratios are plotted in Fig. 2. Circumferential wavenumbers ranging from 1 to 10 are considered here. As is shown this figure, with the increase of the circumferential wavenumber $k$, the general variation trend of frequency parameters decreases first and then increase. Frequency parameters for different boundaries coincide with each other when the circumferential wavenumber $k \geq 3$, and this indicates that the boundary type has a significant influence on frequency parameters corresponding to low circumferential wavenumbers. Furthermore, the frequency parameters increase with the increase of the thickness-radius ratios or decrease with the increase of the length-radius ratios. As can be seen from this figure, the effects of the thickness-radius ratios on the frequency parameters are mainly reflected in the case that the circumferential wavenumber $k \geq 2$, while the effects of the length-radius ratios on the frequency parameters are mainly reflected in the case that the 
circumferential wavenumber $\mathrm{k} \leq 3$.

\subsection{Convergence and validation}

To demonstrate the superiority of the developed approach used in this paper, the convergence of the developed approach is investigated with different number $N$ of grid points along the axial direction. A series of grid points, $N=5,7,9,11$ and 13, is used for this. Fig. 3 shows the influences of grid points on the time-history response of a cylindrical shell at a chosen position $(x=l / 2, \theta=0, z=0)$ and displacements of each point on the $x$-axis at a time when $t=0.26 \mathrm{~s}$. It is evident that the results tend to be stable and consistent when the number of grid points is higher than 7 , which suggests that the convergence rate of the developed approach used in the present work is fast. Moreover, in order to illustrate the merit of the proposed method in improving computation speed, the time required to use the developed method for calculating the dynamic response of the cylindrical shell is compared with the time required to adopt the Navier method. As is shown in Table 4, the time required to use the proposed method for calculating the dynamic response of the cylindrical shell is much less than the time required to the Navier method, and it indicates that the developed method is much more efficient than the Navier method in obtaining the dynamic response of the cylindrical shell.

Table 4 The time required to use different methods for calculating dynamic responses of the cylindrical shell.

\begin{tabular}{lccccc}
\hline \multicolumn{2}{c}{ Methods } & $1^{\text {st }}$ & $2^{\text {nd }}$ & $3^{\text {rd }}$ & Average time (s) \\
\hline NGP (The & 5 & 249.876 & 249.929 & 248.837 & 249.547 \\
present & 7 & 369.504 & 372.406 & 370.019 & 370.718 \\
method) & 9 & 665.313 & 662.661 & 668.121 & 665.365 \\
\hline \multicolumn{2}{r}{ The Navier method } & 1096.38 & 1089.37 & 1092.16 & 1092.64 \\
\hline
\end{tabular}

* NDP: the number of grid points

To prove the effectiveness of the present method, response results of cylindrical shells with SD-SD boundary are also compared with Navier's solution. The displacement components of a cylindrical shell with SD-SD boundary at the ends in terms of the Navier method can be expanded as follows:

$$
\left\{\begin{array}{l}
u(x, \theta, t)=\sum_{n=1}^{\infty} \sum_{m=1}^{\infty} u_{m n}(t) \cos \left(\frac{m \pi x}{l}\right) \cos (n \theta), \\
v(x, \theta, t)=\sum_{n=1}^{\infty} \sum_{m=1}^{\infty} v_{m n}(t) \sin \left(\frac{m \pi x}{l}\right) \sin (n \theta), \\
w(x, \theta, t)=\sum_{n=1}^{\infty} \sum_{m=1}^{\infty} w_{m n}(t) \sin \left(\frac{m \pi x}{l}\right) \cos (n \theta) .
\end{array}\right.
$$

where $u_{m n}(t), v_{m n}(t)$ and $w_{m n}(t)$ represent generalized coordinates, $m$ and $n$ denote the axial half wavenumber and circumferential wavenumber, respectively.

The results of the time-history response of the cylindrical shell with SD-SD boundary at a chosen position $(x=l / 2, \theta=0, z=0)$ obtained by the two methods are compared in Fig. 4 . As shown in this figure, the result derived from the semi-analytical method is in great agreement 
with the Navier's solution.
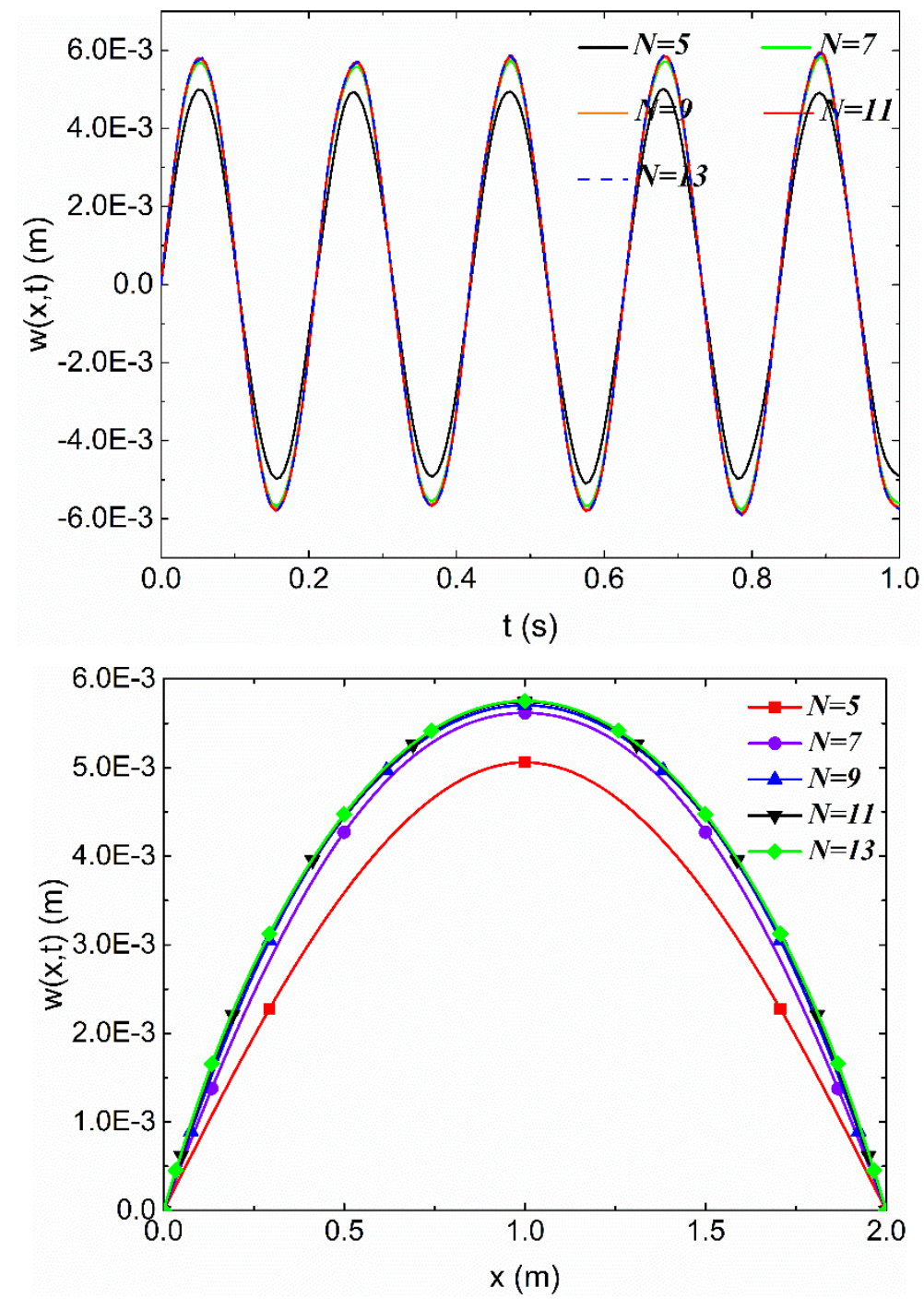

Fig. 3. The time-history curves of a SD-SD cylindrical shell at a chosen position $(x=l / 2, \theta=0$, $z=0)$ under different grid points and displacement of all grid points at a time when $t=0.26 \mathrm{~s}$.

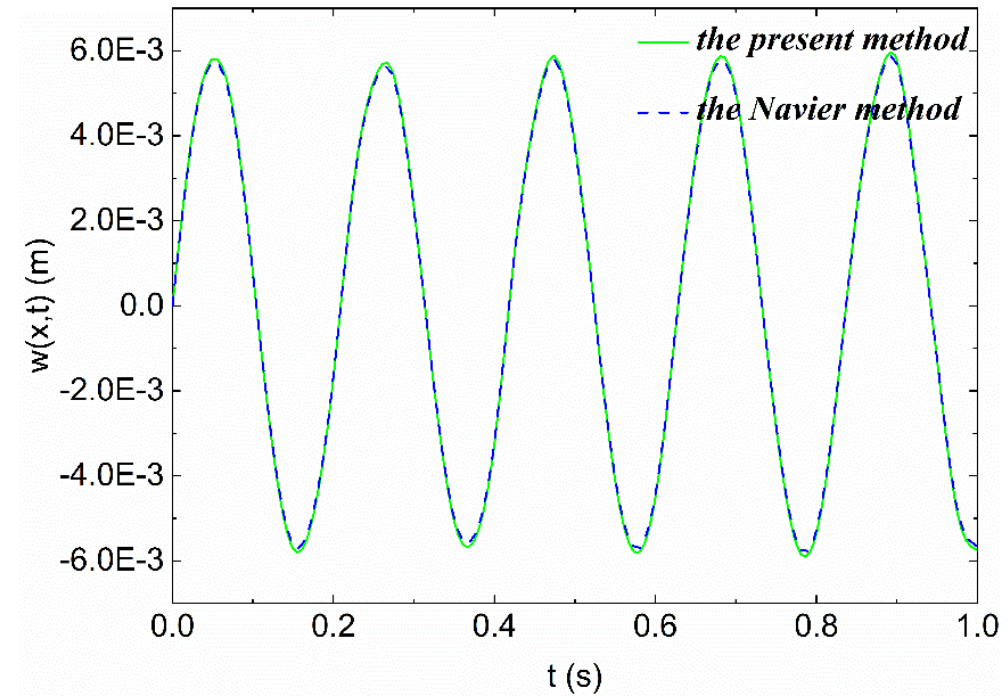

Fig. 4. The time-history curves of a SD-SD cylindrical shell at a chosen position $(x=l / 2, \theta=0$, $z=0)$ using the Navier method and the present method. 


\subsection{Comparison of different shell theories}

The influences of shell theories on the time-history responses of the cylindrical shell are analyzed. The results obtained by classical shell theory (CST) and first-order shear deformation theory (FSDT) with different boundaries and thickness-radius ratios are compared. Two sets of thickness-radius ratios $(h / R=1 / 500,1 / 1000)$ are used to present the time-history responses of cylindrical shells with SD-SD and C-C boundaries. As shown in Fig. 5, the results obtained by CST and FSDT under two sets of thickness-radius ratios are in good agreement, indicating that CST without considering shear deformation and rotary inertia is valid and accurate in calculating time-history responses of the cylindrical shell when the thickness-radius ratios are small enough.
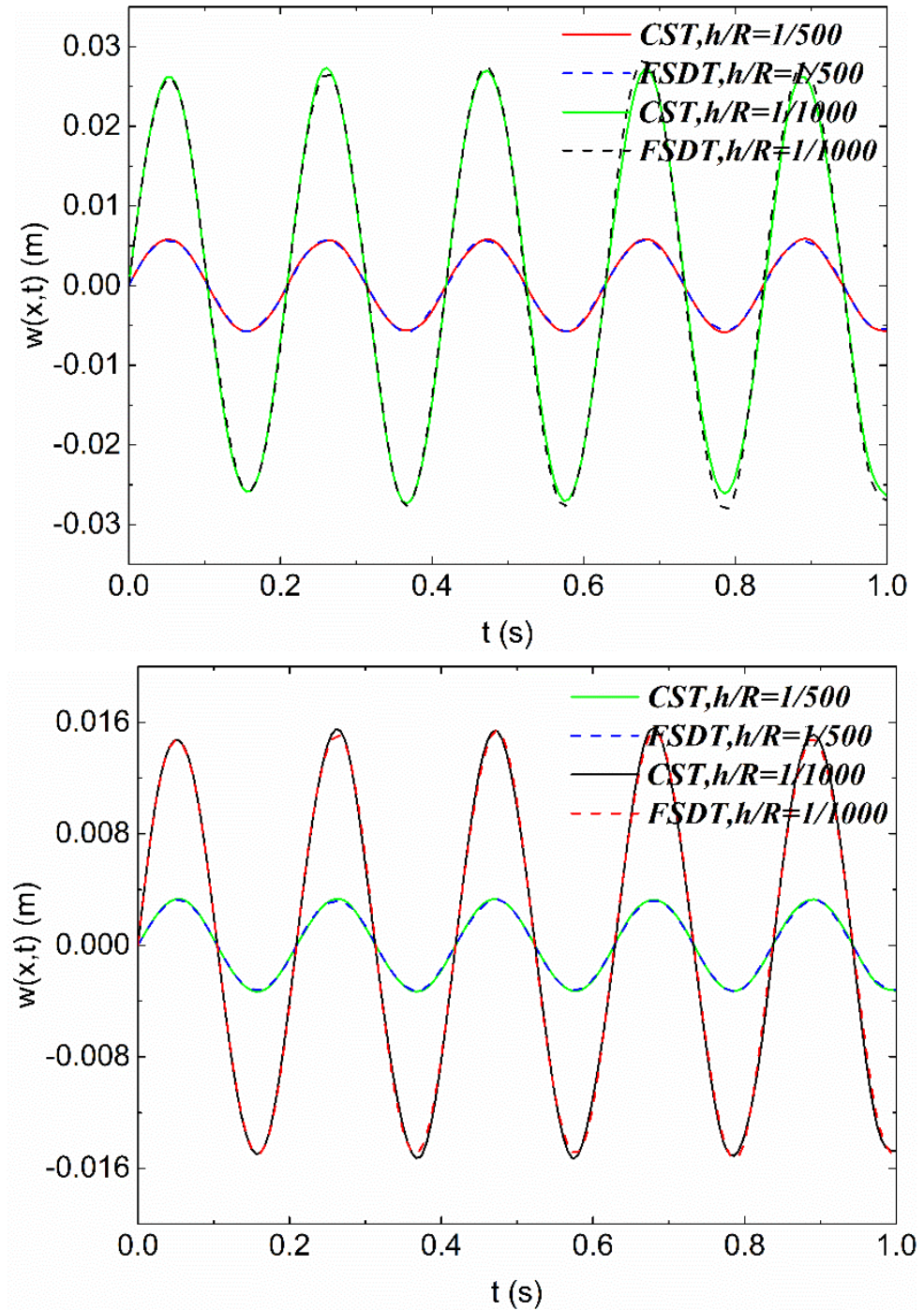

Fig. 5. The time-history curves of the cylindrical shell at a chosen position $(x=l / 2, \theta=0, z=0)$ under SD-SD and C-C boundaries.

\subsection{The influences of boundaries at the edges}

The influences of boundaries at the edges on the time-history response of the cylindrical shell are studied. Four kinds of boundaries at the edges are considered: Simply diaphragm-Simply diaphragm boundaries (SD-SD), Clamped-Clamped boundaries (C-C), Clamped-Simply diaphragm boundaries (C-SD) and Clamped-Free boundaries (C-F). The time-history responses of cylindrical shells under various boundaries at a chosen position 
$(x=l / 2, \theta=0, z=0)$ is plotted in Fig. 6. It is evident that the peak displacement is maximum when the boundary is C-F, and minimum peak displacement of the cylindrical shell appears when the boundary is $\mathrm{C}-\mathrm{C}$, and the results indicate that the peak displacement is closely related to the number of edge degrees of freedom.
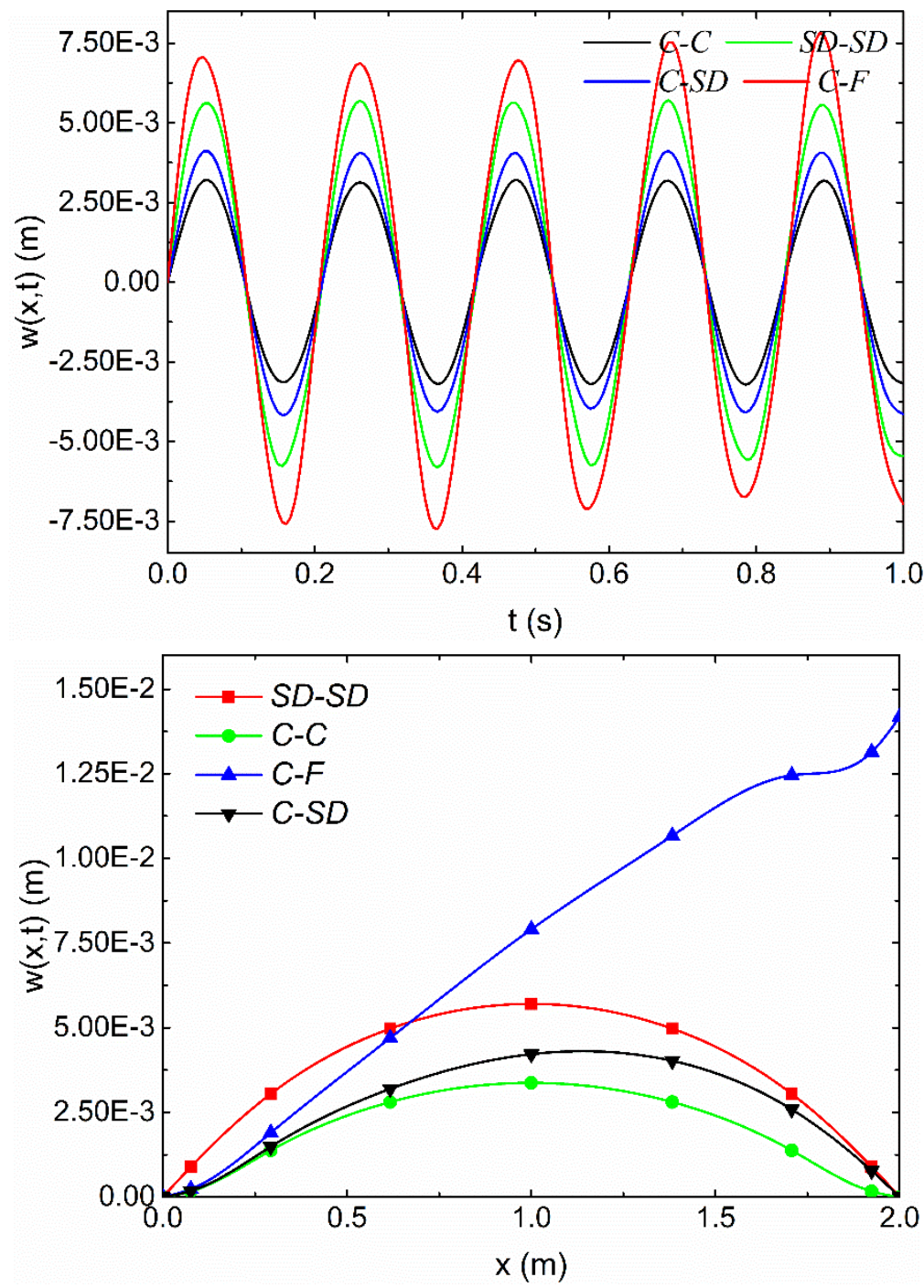

Fig. 6. The time-history curves of a cylindrical shell at a chosen position $(x=l / 2, \theta=0, z=0)$ under different boundaries and displacement of all grid points on the $x$-axis at a time when $t=0.26 \mathrm{~s}$.

\subsection{The influences of geometric dimensioning}

The influences of geometric dimensioning including length-radius ratio $l / R$ and thickness-radius ratio $h / R$ are evaluated. Two sets of geometric dimensioning are used, the first series is: $l / R=1,2,3,4,5$ with $h / R=1 / 250$; the second series is: $h / R=1 / 50,1 / 100,1 / 150$, $1 / 200,1 / 250$ with $l / R=4$. The time-history responses of the cylindrical shell for a given length-radius ratio and thickness-radius ratio at a chosen position $(x=l / 2, \theta=0, z=0)$ are given in Fig. 7. It is found that the peak displacement increases as length-radius ratio increases. On the contrary, for a fixed length-radius ratio, the peak displacement increases with a decrease in thickness-radius ratio. 

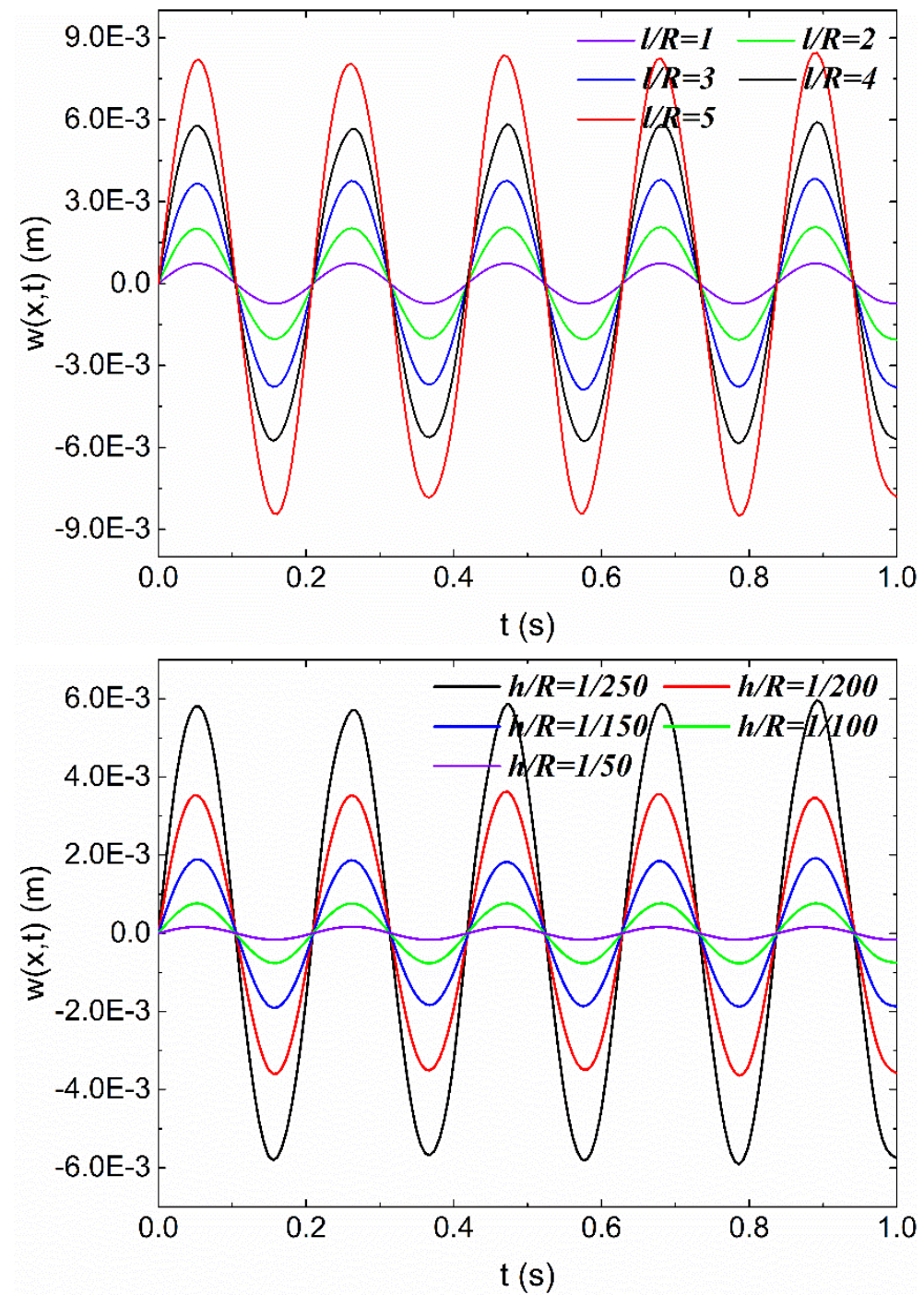

Fig. 7. The time-history responses of a cylindrical shell at a chosen position $(x=l / 2, \theta=0, z=0)$ under different length-radius ratios and thickness-radius ratios.

\section{Conclusions}

In the present work, a novel semi-analytical methodology, which includes the differential quadrature method, the Fourier series expansion technique, and Durbin's numerical inversion method, is proposed to derive the time-history responses of a cylindrical shell under various boundaries.

The frequency parameters of the thin cylindrical shells are calculated and a series of results obtained are compared with the data from the literature. This shows that the results obtained by the semi-analytical approach are consistent with the results in the literature. To demonstrate the convergence and the validity of the results obtained, varying numbers of grid points along the axial direction are used and a comparison of results with the Navier's solution is carried out. The results indicate that the approach used in this paper is of fast rapidity of convergence and high accuracy. A comparison of time-history responses derived by classical shell theory with results obtained by first-order shear deformation theory shows that the classical shell theory is accurate and precise in estimating the time-history responses of cylindrical shells when the thickness-radius ratio is small enough. The analysis of various 
boundaries on the time-history response of cylindrical shells shows that the peak displacement of cylindrical shells has a close relationship with the number of edge degrees of freedom. The influences of length-radius ratios and thickness-radius ratios on the time-history responses of cylindrical shells are also analyzed. Parametric studies show that the peak displacement becomes larger as the length-radius ratio increases or the thickness-radius ratio decreases.

\section{Acknowledgements}

This work was supported by the National Natural science Foundation of China (Grant Nos. 51879231, 51679214, 51409228), and the authors would like to express sincere gratitude to Victor Sun Zheng for his assistance in editing this manuscript.

\section{Appendix A}

$$
\begin{aligned}
& \mathbf{H}_{11}=A_{11} R \mathbf{A}_{i j}^{(2)}+\left(-\frac{A_{66} k^{2}}{R}-I_{0} R s^{2}\right) \mathbf{I}_{N}, \mathbf{H}_{12}=\left(A_{12} k+A_{66} k+\frac{B_{12} k}{R}+\frac{2 B_{66} k}{R}\right) \mathbf{A}_{i j}^{(1)}, \\
& \mathbf{H}_{13}=\left(A_{12}+\frac{B_{12} k^{2}}{R}+\frac{2 B_{66} k^{2}}{R}\right) \mathbf{A}_{i j}^{(1)}-B_{11} R \mathbf{A}_{i j}^{(3)}, \mathbf{H}_{21}=\left(-A_{12} k-A_{66} k-\frac{B_{12} k}{R}-\frac{2 B_{66} k}{R}\right) \mathbf{A}_{i j}^{(1)}, \\
& \mathbf{H}_{22}=\left(4 B_{66}+\frac{4 D_{66}}{R}+A_{66} R\right) \mathbf{A}_{i j}^{(2)}+\left(-\frac{D_{22} k^{2}}{R^{3}}-\frac{2 B_{22} k^{2}}{R^{2}}-\frac{A_{22} k^{2}}{R}-I_{0} R s^{2}\right) \mathbf{I}_{N}, \\
& \mathbf{H}_{23}=\left(B_{12} k+2 B_{66} k+\frac{D_{12} k}{R}+\frac{4 D_{66} k}{R}\right) \mathbf{A}_{i j}^{(2)}+\left(-\frac{D_{22} k^{3}}{R^{3}}-\frac{B_{22} k}{R^{2}}-\frac{B_{22} k^{3}}{R^{2}}-\frac{A_{22} k}{R}\right) \mathbf{I}_{N}, \\
& \mathbf{H}_{31}=\left(-A_{12}-\frac{B_{12} k^{2}}{R}-\frac{2 B_{66} k^{2}}{R}\right) \mathbf{A}_{i j}^{(1)}+B_{11} R \mathbf{A}_{i j}^{(3)}, \\
& \mathbf{H}_{32}=\left(B_{12} k+2 B_{66} k+\frac{D_{12} k}{R}+\frac{4 D_{66} k}{R}\right) \mathbf{A}_{i j}^{(2)}+\left(-\frac{D_{22} k^{3}}{R^{3}}-\frac{B_{22} k}{R^{2}}-\frac{B_{22} k^{3}}{R^{2}}-\frac{A_{22} k}{R}\right) \mathbf{I}_{N}, \\
& \mathbf{H}_{33}=\left(2 B_{12}+\frac{2 D_{12} k^{2}}{R}+\frac{4 D_{66} k^{2}}{R}\right) \mathbf{A}_{i j}^{(2)}-D_{11} R \mathbf{A}_{i j}^{(4)}+\left(-\frac{D_{22} k^{4}}{R^{3}}-\frac{2 B_{22} k^{2}}{R^{2}}-\frac{A_{22}}{R}-I_{0} R s^{2}\right) \mathbf{I}_{N} . \\
& I_{N}=\left[\begin{array}{cccc}
1 & 0 & \cdots & 0 \\
0 & 1 & \cdots & 0 \\
\vdots & \vdots & \ddots & \vdots \\
0 & 0 & \cdots & 1
\end{array}\right]_{N \times N}, \mathbf{A}_{i j}^{(m)}=\left[\begin{array}{cccc}
A_{11}^{(m)} & A_{12}^{(m)} & \cdots & A_{1 N}^{(m)} \\
A_{21}^{(m)} & A_{22}^{(m)} & \cdots & A_{2 N}^{(m)} \\
\vdots & \vdots & \ddots & \vdots \\
A_{N 1}^{(m)} & A_{N 2}^{(m)} & \cdots & A_{N N}^{(m)}
\end{array}\right] .
\end{aligned}
$$

\section{Appendix B}

$$
\begin{aligned}
& H_{11,1 i}=T_{11} A_{1 i}^{(1)}, i=2 \sim N, H_{11,11}=T_{11} A_{11}^{(1)}-k_{u 0}, \\
& H_{11, N i}=-T_{11} A_{N i}^{(1)}, i=1 \sim N-1, H_{11, N N}=-T_{11} A_{N N}^{(1)}-k_{u L}, \\
& H_{12,1 i}=0, i=2 \sim N, H_{12,11}=T_{12}, \\
& H_{12, N i}=0, i=1 \sim N-1, H_{12, N N}=-T_{12}, \\
& H_{13,1 i}=0, i=2 \sim N, H_{13,11}=T_{13}, \\
& H_{13, N i}=0, i=1 \sim N-1, H_{13, N N}=-T_{13} .
\end{aligned}
$$




$$
\begin{aligned}
& H_{21,1 i}=0, i=2 \sim N, H_{21,11}=T_{21}, \\
& H_{21, N i}=0, i=1 \sim N-1, H_{21, N N}=-T_{21}, \\
& H_{22,1 i}=T_{22} A_{1 i}^{(1)}, i=2 \sim N, H_{22,11}=T_{22} A_{11}^{(1)}-k_{v 0}, \\
& H_{22, N i}=-T_{22} A_{N i}^{(1)}, i=1 \sim N-1, H_{22, N N}=-T_{22} A_{N N}^{(1)}-k_{v L}, \\
& H_{23,1 i}=T_{23} A_{1 i}^{(1)}, H_{23, N i}=-T_{23} A_{N i}^{(1)}, i=1 \sim N . \\
& H_{31,1 i}=0, H_{31, N i}=0, i=1 \sim N, \\
& H_{32,1 i}=T_{32} A_{1 i}^{(1)}, i=2 \sim N-1, H_{32,11}=T_{32} A_{11}^{(1)}, \\
& H_{32, N i}=-T_{32} A_{N i}^{(1)}, i=1 \sim N-1, H_{32, N N}=-T_{32} A_{N N}^{(1)}, \\
& H_{33,1 i}=T_{331} A_{1 i}^{(1)}+T_{332} A_{1 i}^{(3)}, i=2 \sim N, \\
& H_{33,11}=T_{331} A_{11}^{(1)}+T_{332} A_{11}^{(3)}-k_{w 0}, \\
& H_{33, N i}=-T_{331} A_{N i}^{(1)}-T_{332} A_{N i}^{(3)}, i=1 \sim N-1, \\
& H_{33, N N}=-T_{331} A_{N N}^{(1)}-T_{332} A_{N N}^{(3)}-k_{w L} . \\
& H_{31,2 i}=0, H_{31, N-1, i}=0, i=1 \sim N, \\
& H_{32,2 i}=0, i=2 \sim N-1, H_{32,21}=T_{42}, \\
& H_{32, N-1, i}=0, i=1 \sim N-1, H_{32, N-1, N}=-T_{42}, \\
& H_{33,2 i}=T_{432} A_{1 i}^{(2)}-K_{w 0} A_{1 i}^{(1)}, i=2 \sim N, \\
& H_{33,21}=T_{432} A_{11}^{(2)}-K_{w 0} A_{11}^{(1)}+T_{431}, \\
& H_{33, N-1, i}=-T_{432} A_{N i}^{(2)}-K_{w 0} A_{N i}^{(1)}, i=1 \sim N-1, \\
& H_{33, N-1, N}=-T_{432} A_{N N}^{(2)}-K_{w 0} A_{N N}^{(1)}-T_{431} .
\end{aligned}
$$

\section{References}

Bodaghi, M., Shakeri, M., 2012. An analytical approach for free vibration and transient response of functionally graded piezoelectric cylindrical panels subjected to impulsive loads. Composite Structures 94 (5), 1721-1735.

Civalek, Ö., 2006. The determination of frequencies of laminated conical shells via the discrete singular convolution method. Journal of Mechanics of Materials \& Structures 1 (1), 163-182.

Civalek, Ö., 2013. Vibration analysis of laminated composite conical shells by the method of discrete singular convolution based on the shear deformation theory. Composites Part B Engineering 45 (1), 1001-1009.

Duc, N.D., 2013. Nonlinear dynamic response of imperfect eccentrically stiffened FGM double curved shallow shells on elastic foundation. Composite Structures 99, 88-96.

Durbin, F., 1974. Numerical inversion of Laplace Transforms: An efficient improvement to Dubner and Abate's method. Computer Journal 17 (4), 371-376.

Ferreira, A.J.M., Carrera, E., Cinefra, M., Roque, C.M.C., Polit, O., 2011. Analysis of laminated shells by a sinusoidal shear deformation theory and radial basis functions collocation, accounting for through-the-thickness deformations. Composites Part B Engineering 42 (5), 1276-1284.

Ferreira, A.J.M., Roque, C.M.C., Jorge, R.M.N., 2006. Modelling cross-ply laminated elastic shells by a higher-order theory and multiquadrics. Computers \& Structures 84 (19), 
1288-1299.

Frikha, A., Zghal, S., Dammak, F., 2018. Dynamic analysis of functionally graded carbon nanotubes-reinforced plate and shell structures using a double directors finite shell element. Aerospace Science \& Technology 78, 438-451.

Gu, J., An, C., Duan, M., Levi, C., Su, J., 2013. Integral transform solutions of dynamic response of a clamped-clamped pipe conveying fluid. Nuclear Engineering \& Design 254 (1), 237-245.

Gupta, S., Matos, H., Leblanc, J.M., Shukla, A., 2016. Shock initiated instabilities in underwater cylindrical structures. Journal of the Mechanics \& Physics of Solids 95, 188-212. Haddadpour, H., Mahmoudkhani, S., Navazi, H.M., 2007. Free vibration analysis of functionally graded cylindrical shells including thermal effects. Thin-Walled Structures 45 (6), 591-599.

Hajmohammad, M.H., Kolahchi, R., Zarei, M.S., Maleki, M., 2017. Earthquake induced dynamic deflection of submerged viscoelastic cylindrical shell reinforced by agglomerated CNTs considering thermal and moisture effects. Composite Structures 187, 498-508.

Jin, G., Yang, C., Liu, Z., Gao, S., Zhang, C., 2015. A unified method for the vibration and damping analysis of constrained layer damping cylindrical shells with arbitrary boundary conditions. Composite Structures 130, 124-142.

Jin, G., Ye, T., Ma, X., Chen, Y., Su, Z., Xie, X., 2013. A unified approach for the vibration analysis of moderately thick composite laminated cylindrical shells with arbitrary boundary conditions. International Journal of Mechanical Sciences 75 (10), 357-376.

Kadoli, R., Ganesan, N., 2006. Buckling and free vibration analysis of functionally graded cylindrical shells subjected to a temperature-specified boundary condition. Journal of Sound \& Vibration 289 (3), 450-480.

Lam, K.Y., Loy, C.T., 1995. Effects of boundary conditions on frequencies of a multi-layered cylindrical shell. Journal of Sound \& Vibration 188 (188), 363-384.

Leissa, A.W., 1993. Vibration of shells. US Government Printing Office, Washington DC.

Liang, S., Chen, H.L., 2006. The natural vibration of a conical shell with an annular end plate. Journal of Sound \& Vibration 294 (4), 927-943.

Liang, X., Wang, Z., Wang, L., Liu, G., 2014. Semi-analytical solution for three-dimensional transient response of functionally graded annular plate on a two parameter viscoelastic foundation. Journal of Sound \& Vibration 333 (12), 2649-2663.

Liu, J., He, W., Xie, D., 2018. Study on Vibrational Power Flow Propagation Characteristics in a Laminated Composite Cylindrical Shell Filled with Fluid. Shock \& Vibration 2018 (4), $1-19$.

Loy, C.T., Lam, K.Y., Shu, C., 1997. Analysis of cylindrical shells using generalized differential quadrature. Shock \& Vibration 4 (3), 193-198.

Lu, C., Lam, K.Y., 1995. Dynamic analysis of clamped laminated curved panels. Composite Structures 30 (4), 389-398.

Maleki, S., Tahani, M., Andakhshideh, A., 2012. Static and transient analysis of laminated cylindrical shell panels with various boundary conditions and general lay - ups. ZAMM Journal of Applied Mathematics and Mechanics / Zeitschrift für Angewandte Mathematik und Mechanik 92 (2), 124-140.

Markuš, Š., 1988. The mechanics of vibrations of cylidrical shells. Elsevier, New York. 
Pradhan, S.C., Loy, C.T., Lam, K.Y., Reddy, J.N., 2000. Vibration characteristics of functionally graded cylindrical shells under various boundary conditions. Applied Acoustics 61 (1), 111-129.

Qatu, M.S., 2004. Vibration of laminated shells and plates. Elsevier, San Diego.

Qing, G., Liu, Y., Guo, Q., Zhang, D., 2008. Dynamic analysis for three-dimensional laminated plates and panels with damping. International Journal of Mechanical Sciences 50 (1), 83-91.

Qu, Y., Hua, H., Meng, G., 2013a. A domain decomposition approach for vibration analysis of isotropic and composite cylindrical shells with arbitrary boundaries. Composite Structures 95, 307-321.

Qu, Y., Long, X., Wu, S., Meng, G., 2013b. A unified formulation for vibration analysis of composite laminated shells of revolution including shear deformation and rotary inertia. Composite Structures 98 (3), 169-191.

Reddy, J.N., 2003. Mechanics of laminated composite plates and shells : theory and analysis. CRC Press, Florida.

Reddy, J.N., Khdeir, A.A., 1989. Dynamic response of cross - ply laminated shallow shells according to a refined shear deformation theory. Journal of the Acoustical Society of America 85 (6), 2423-2431.

Santos, H., Soares, C.M.M., Soares, C.A.M., Reddy, J.N., 2009. A semi-analytical finite element model for the analysis of cylindrical shells made of functionally graded materials. Composite Structures 91 (4), 427-432.

Shao, D., Hu, S., Wang, Q., Pang, F., 2017a. An enhanced reverberation-ray matrix approach for transient response analysis of composite laminated shallow shells with general boundary conditions. Composite Structures 162, 133-155.

Shao, D., Wang, Q., Qin, B., 2017b. A simple first-order shear deformation shell theory for vibration analysis of composite laminated open cylindrical shells with general boundary conditions. Composite Structures.

Thai, H.T., Kim, S.E., 2015. A review of theories for the modeling and analysis of functionally graded plates and shells. Composite Structures 128 (3), 70-86.

Wu, J., Chong, J., Long, Y., Zhou, Y., Yu, Y., Liu, J., 2018. Experimental study on the deformation and damage of cylindrical shell-water-cylindrical shell structures subjected to underwater explosion. Thin-Walled Structures 127, 654-665.

Yazdi, A.A., 2013. Applicability of homotopy perturbation method to study the nonlinear vibration of doubly curved cross-ply shells. Composite Structures 96 (96), 526-531.

Zhang, L.W., Song, Z.G., Qiao, P., Liew, K.M., 2017. Modeling of dynamic responses of CNT-reinforced composite cylindrical shells under impact loads. Computer Methods in Applied Mechanics \& Engineering 313, 889-903.

Zhang, Y., Duan, M., Yi, W., Gui, C., 2015a. Analytical study of the strength of adhesive joints of riser pipes. Ships \& Offshore Structures 10 (5), 545-553.

Zhang, Y., Wu, P., Duan, M., 2015b. A mesh-independent technique to evaluate stress singularities in adhesive joints. International Journal of Adhesion \& Adhesives 57, 105-117. 


\section{List of Tables}

Table 1 Frequency parameters of a thin cylindrical shell with three different boundaries $(h / R=0.01)$.

Table 2 Frequency parameters of SD-SD cylindrical shells.

Table 3 Frequency parameters of C-SD cylindrical shells $(h / R=0.002)$.

Table 4 The time required to use different methods for calculating dynamic responses of the cylindrical shell. 
Table 1 Frequency parameters of a thin cylindrical shell with three different boundaries $(h / R=0.01)$.

\begin{tabular}{ccccccc}
\hline & \multicolumn{5}{c}{ Frequency parameters $\Omega=\omega R \sqrt{\left(1-\mu^{2}\right) \rho / E}$} \\
\cline { 2 - 7 }$k$ & \multicolumn{2}{c}{ SD-SD } & \multicolumn{2}{c}{ C-C } & \multicolumn{3}{c}{ C-SD } \\
\cline { 2 - 7 } & $\begin{array}{c}\text { Loy et al. } \\
(1997)\end{array}$ & Present & $\begin{array}{c}\text { Loy et al. } \\
(1997)\end{array}$ & Present & $\begin{array}{c}\text { Loy et al. } \\
(1997)\end{array}$ & Present \\
\hline 1 & 0.016101 & 0.016101 & 0.032885 & 0.032884 & 0.023974 & 0.023976 \\
2 & 0.009382 & 0.009378 & 0.013923 & 0.013929 & 0.011225 & 0.011221 \\
3 & 0.022105 & 0.022103 & 0.022672 & 0.022668 & 0.022310 & 0.022308 \\
4 & 0.042095 & 0.042094 & 0.042208 & 0.042207 & 0.042139 & 0.042137 \\
5 & 0.068008 & 0.068007 & 0.068046 & 0.068045 & 0.068024 & 0.068023 \\
6 & 0.099730 & 0.099729 & 0.099748 & 0.099747 & 0.099738 & 0.099737 \\
7 & 0.137239 & 0.137238 & 0.137249 & 0.137249 & 0.137244 & 0.137243 \\
8 & 0.180527 & 0.180527 & 0.180535 & 0.180534 & 0.180531 & 0.180531 \\
9 & 0.229594 & 0.229593 & 0.229599 & 0.229598 & 0.229596 & 0.229596 \\
10 & 0.284435 & 0.284435 & 0.284439 & 0.284439 & 0.284437 & 0.284437 \\
\hline
\end{tabular}


Table 2 Frequency parameters of SD-SD cylindrical shells.

\begin{tabular}{ccccccc}
\hline \multicolumn{8}{c}{ Frequency parameters $\Omega=\omega R \sqrt{\left(1-\mu^{2}\right) \rho / E}$} \\
\hline \multirow{3}{*}{$k$} & \multicolumn{3}{c}{$h / R=0.05$} & & \multicolumn{3}{c}{$h / R=0.002$} \\
\cline { 2 - 7 } & Markuš & Loy et al. & Present & Markuš & Loy et al. & Present \\
\hline 1 & 0.0161063 & 0.01610 & 0.0161029 & 0.0161011 & 0.016101 & 0.0161011 \\
2 & 0.0392332 & 0.03930 & 0.0392710 & 0.00545243 & 0.005453 & 0.00545297 \\
3 & 0.109477 & 0.109824 & 0.1098116 & 0.00503724 & 0.005042 & 0.00504148 \\
4 & 0.209008 & 0.210284 & 0.2102773 & 0.00853409 & 0.008534 & 0.00853382 \\
\hline
\end{tabular}


Table 3 Frequency parameters of C-SD cylindrical shells $(h / R=0.002)$.

Frequency parameters $\Omega=\omega R \sqrt{\left(1-\mu^{2}\right) \rho / E}$

\begin{tabular}{llllccccc}
\hline & $\begin{array}{l}\text { Lam and } \\
k\end{array}$ & Loy \\
$(1995)$ & $\begin{array}{c}\text { Loy et al. } \\
(1997)\end{array}$ & Present & $k$ & $\begin{array}{c}\text { Lam and } \\
\text { Loy }\end{array}$ & $\begin{array}{c}\text { Loy et al. } \\
(1997)\end{array}$ & Present \\
& & & & $(1995)$ & \\
\hline 1 & 0.024830 & 0.023974 & 0.023976 & 6 & 0.019974 & 0.019973 & 0.019972 \\
2 & 0.008410 & 0.008223 & 0.008222 & 7 & 0.027461 & 0.027460 & 0.027460 \\
3 & 0.005897 & 0.005842 & 0.005842 & 8 & 0.036113 & 0.36112 & 0.036112 \\
4 & 0.008717 & 0.008705 & 0.008705 & 9 & 0.045924 & 0.045923 & 0.045923 \\
5 & 0.013682 & 0.013679 & 0.013679 & 10 & 0.056891 & 0.056890 & 0.056890 \\
\hline
\end{tabular}


Table 4 The time required to use different methods for calculating dynamic responses of the cylindrical shell.

\begin{tabular}{lccccc}
\hline \multicolumn{2}{c}{ Methods } & $1^{\text {st }}$ & $2^{\text {nd }}$ & $3^{\text {rd }}$ & Average time (s) \\
\hline NGP (The & 5 & 249.876 & 249.929 & 248.837 & 249.547 \\
present & 7 & 369.504 & 372.406 & 370.019 & 370.718 \\
method) & 9 & 665.313 & 662.661 & 668.121 & 665.365 \\
\hline \multicolumn{2}{r}{ The Navier method } & 1096.38 & 1089.37 & 1092.16 & 1092.64 \\
\hline
\end{tabular}

* NDP: the number of grid points 


\section{List of Figures}

Fig. 1. Geometry of the thin cylindrical shell subjected to a harmonic load.

Fig. 2. Variations of frequency parameters versus circumferential wavenumber $k$ : (a) different boundaries; (b) different thickness-radius ratios $h / R$; (c) different length-radius ratios $l / R$.

Fig. 3. The time-history curves of a SD-SD cylindrical shell at a chosen position $(x=l / 2, \theta=0$, $z=0)$ under different grid points and displacement of all grid points at a time when $t=0.26 \mathrm{~s}$.

Fig. 4 . The time-history curves of a SD-SD cylindrical shell at a chosen position $(x=l / 2, \theta=0$, $z=0$ ) using the Navier method and the present method.

Fig. 5. The time-history curves of the cylindrical shell at a chosen position $(x=l / 2, \theta=0, z=0)$ under SD-SD and C-C boundaries.

Fig. 6. The time-history curves of a cylindrical shell at a chosen position $(x=l / 2, \theta=0, z=0)$ under different boundaries and displacement of all grid points on the $x$-axis at a time when $t=0.26 \mathrm{~s}$.

Fig. 7. The time-history responses of a cylindrical shell at a chosen position $(x=l / 2, \theta=0, z=0)$ under different length-radius ratios and thickness-radius ratios. 
1

2

3

4

5

6

7

8

10

11

12

13

14

15

16

17

18

19

20

21

22

23

24

25

26

27

28

29

30

31

32

33

34

35

36

37

38

39

40

41

42

43

44

45

46

47

48

49

50

51

52

53

54

55

56

57

58

59

60

61

62

63

64

65

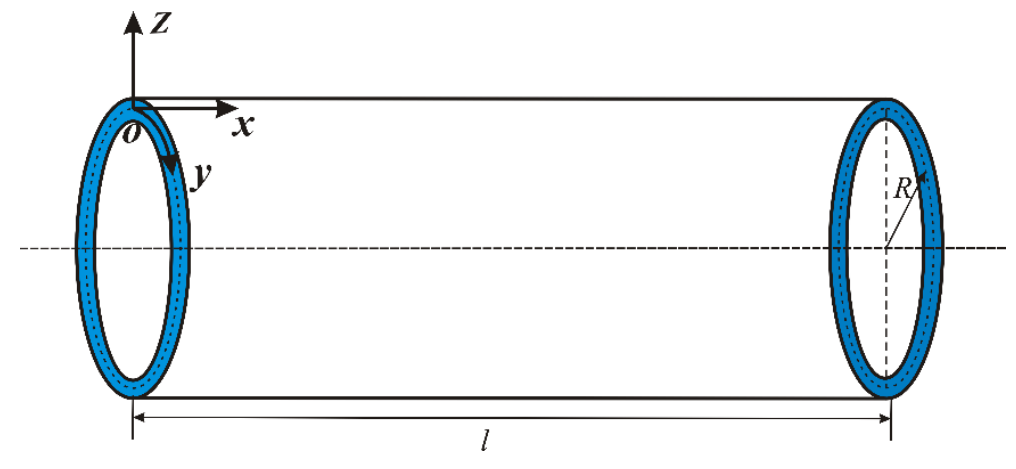

Fig. 1. Geometry of the thin cylindrical shell subjected to a harmonic load. 


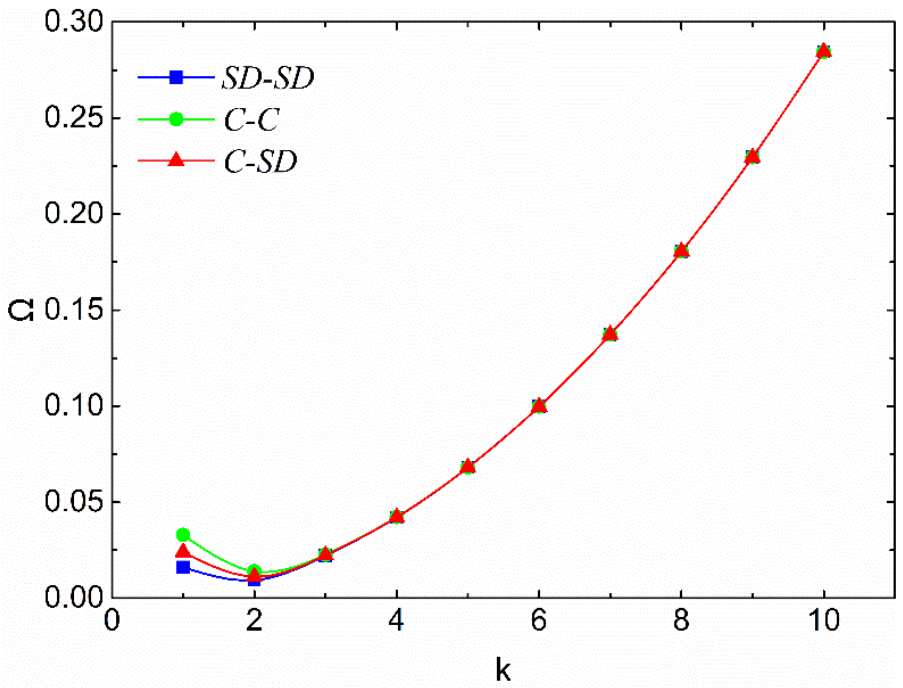

(a) different boundaries

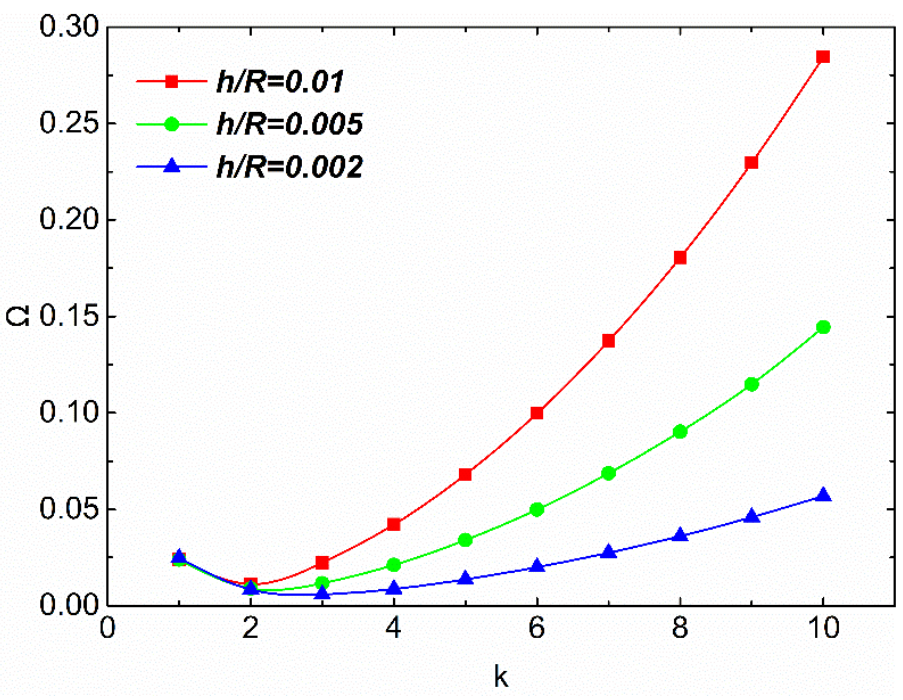

(b) different thickness-radius ratios $h / R$

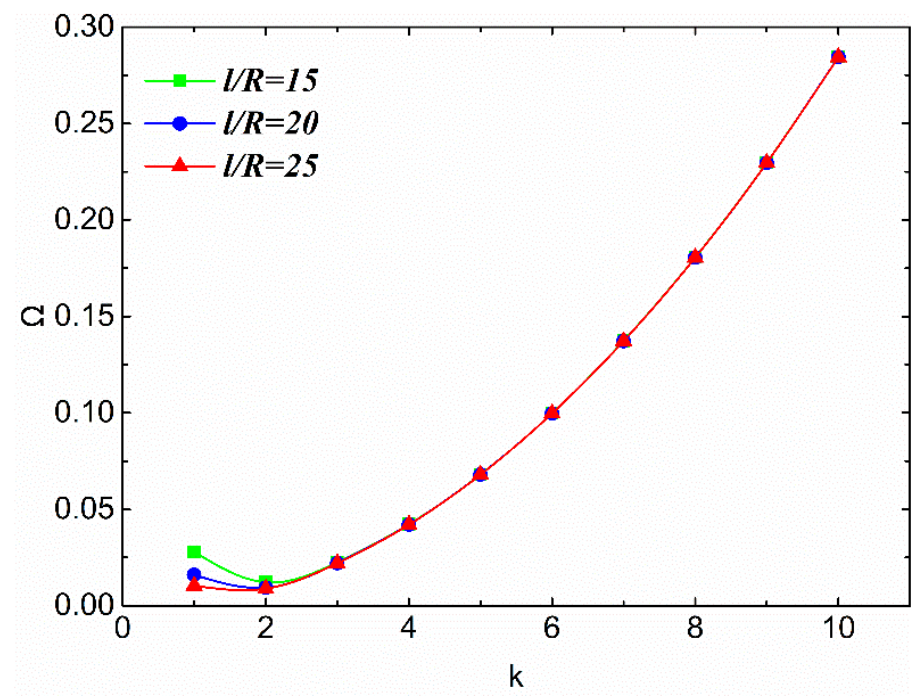

(c) different length-radius ratios $l / R$

Fig. 2. Variations of frequency parameters versus circumferential wavenumber $k$ : (a) different boundaries; (b) different thickness-radius ratios $h / R$.; (c) different length-radius ratios $l / R$. 

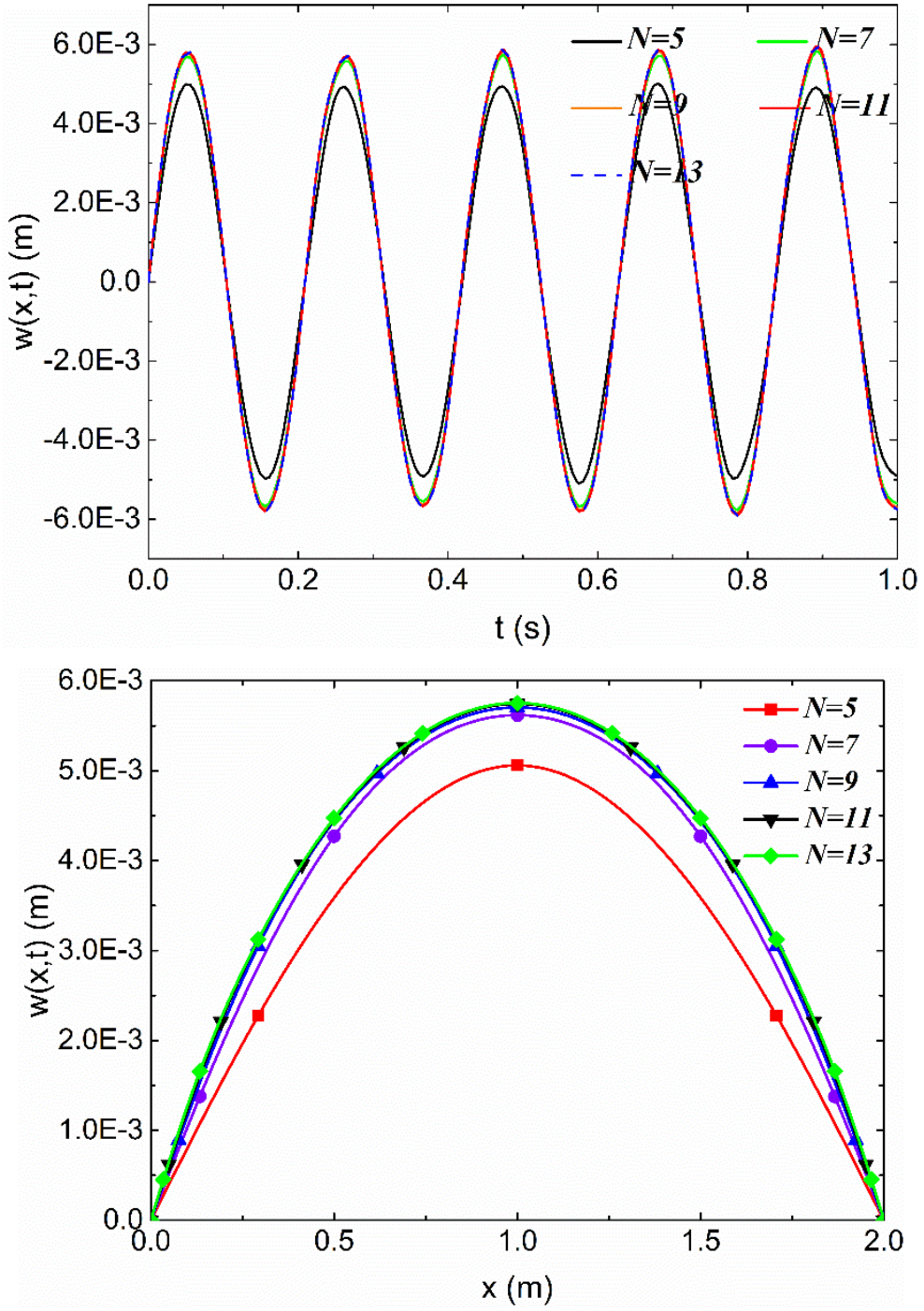

Fig. 3. The time-history curves of a SD-SD cylindrical shell at a chosen position $(x=l / 2, \theta=0$, $z=0)$ under different grid points and displacement of all grid points at a time when $t=0.26 \mathrm{~s}$. 


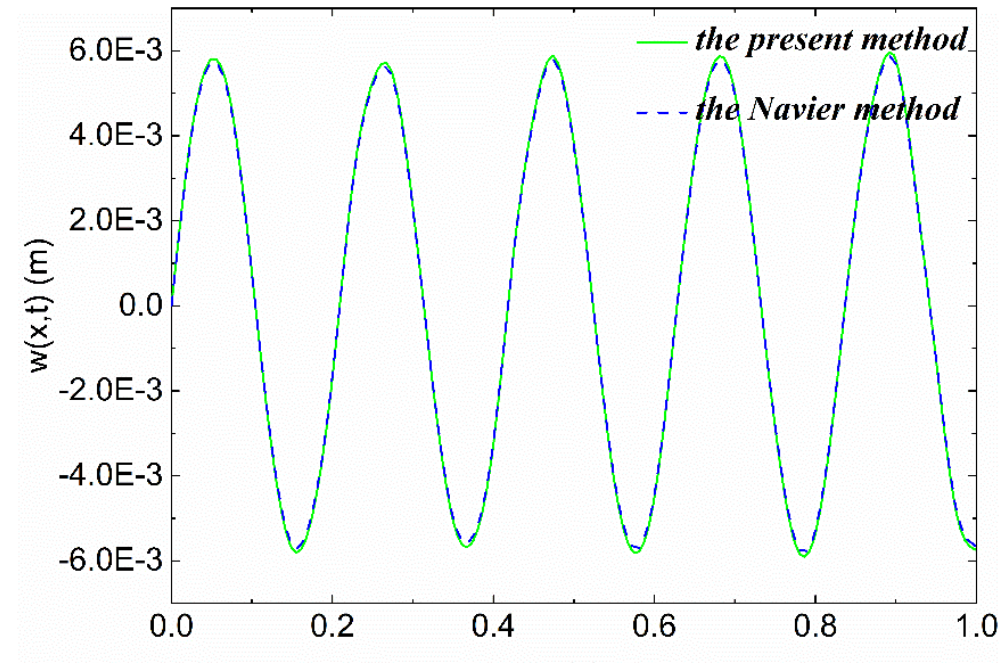

$t(s)$

Fig. 4. The time-history curves of a SD-SD cylindrical shell at a chosen position $(x=l / 2, \theta=0$, $z=0$ ) using the Navier method and the present method. 


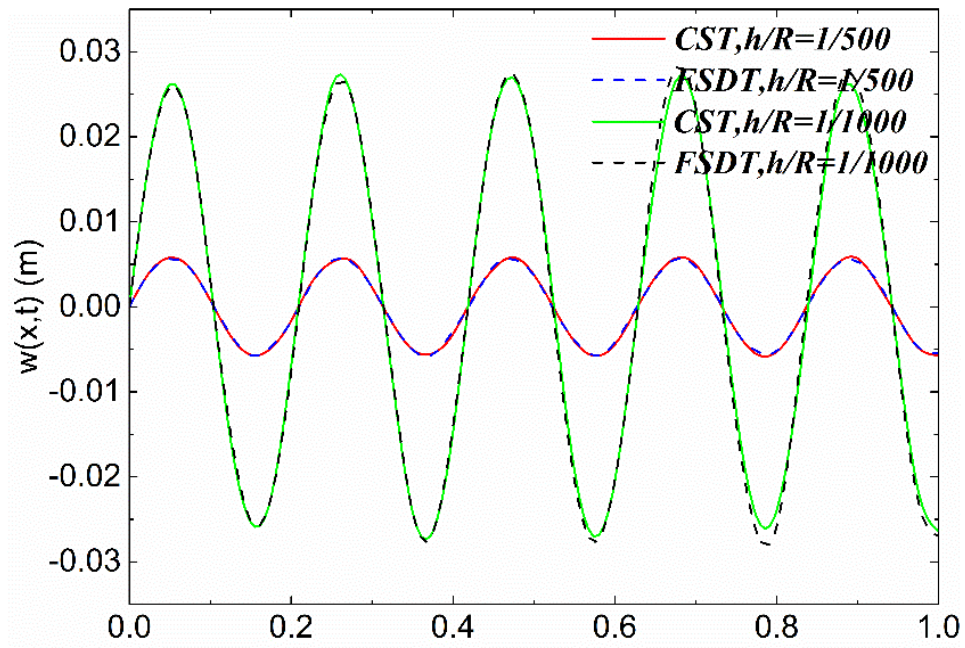

$t$ (s)

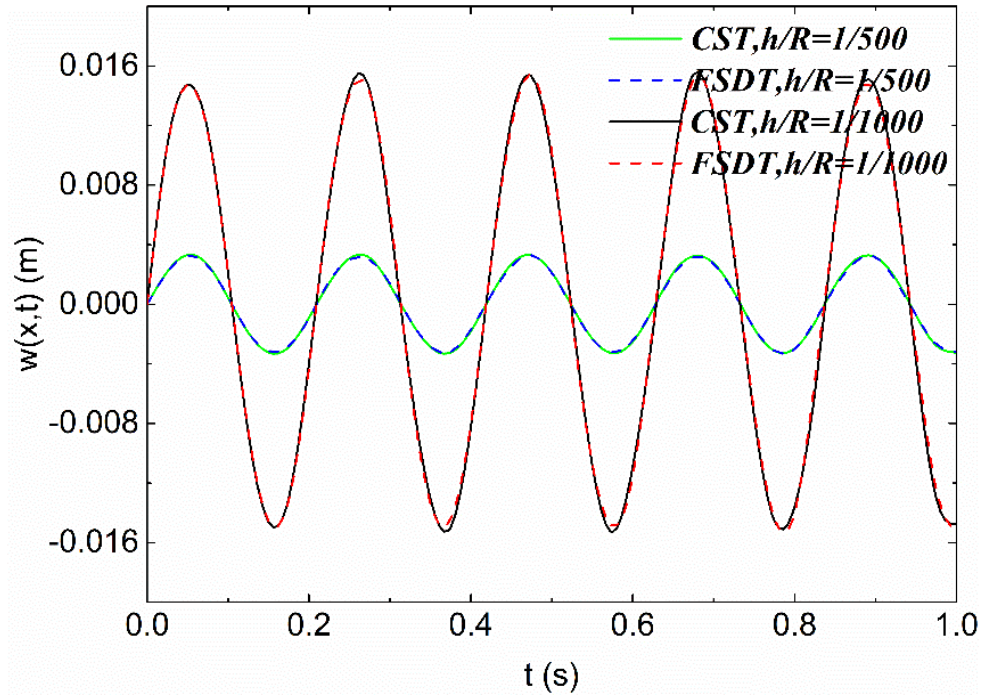

Fig. 5. The time-history curves of the cylindrical shell at a chosen position $(x=l / 2, \theta=0, z=0)$ under SD-SD and C-C boundaries. 

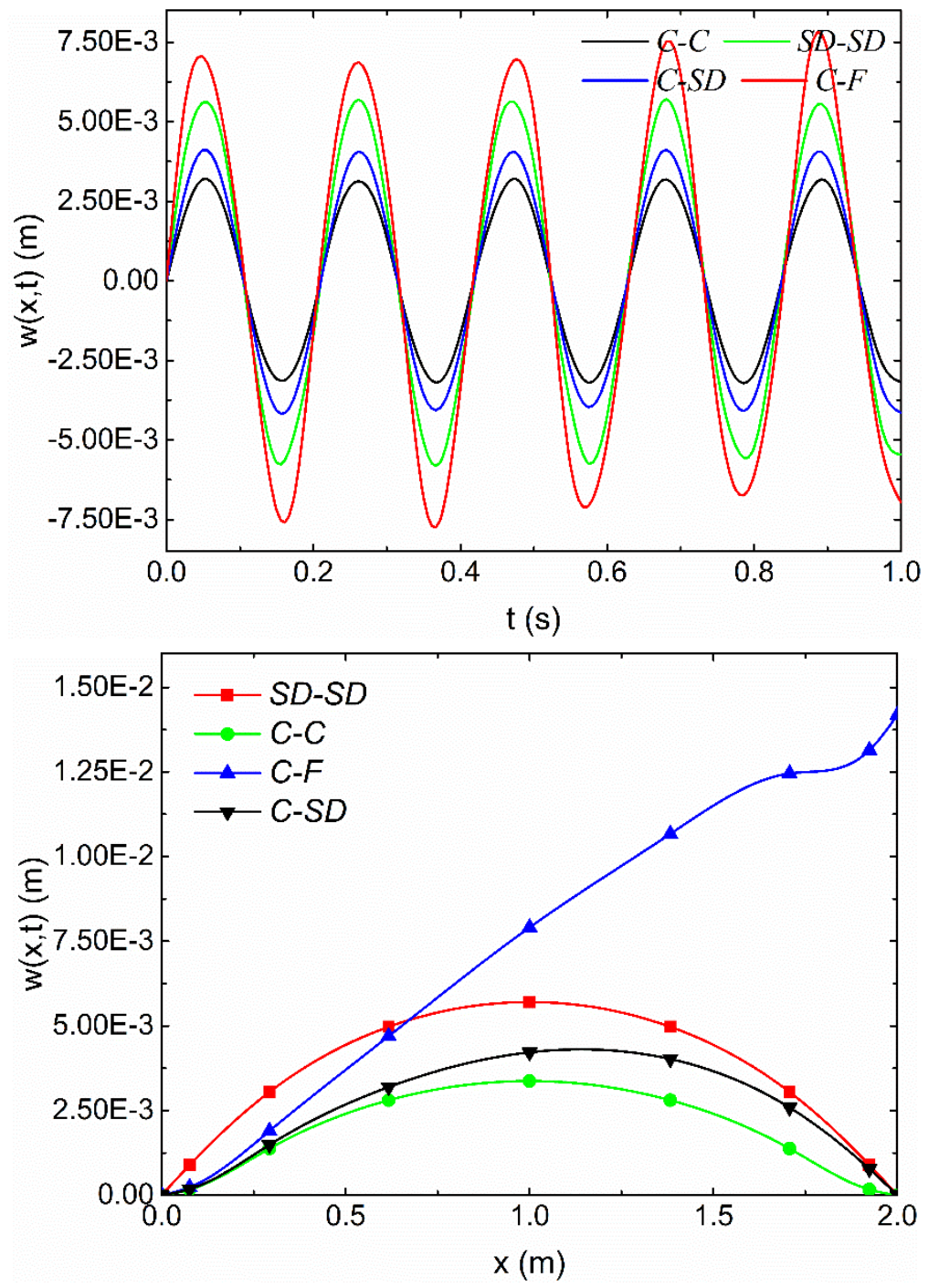

Fig. 6. The time-history curves of a cylindrical shell at a chosen position $(x=l / 2, \theta=0, z=0)$ under different boundaries and displacement of all grid points on the $x$-axis at a time when $t=0.26 \mathrm{~s}$. 

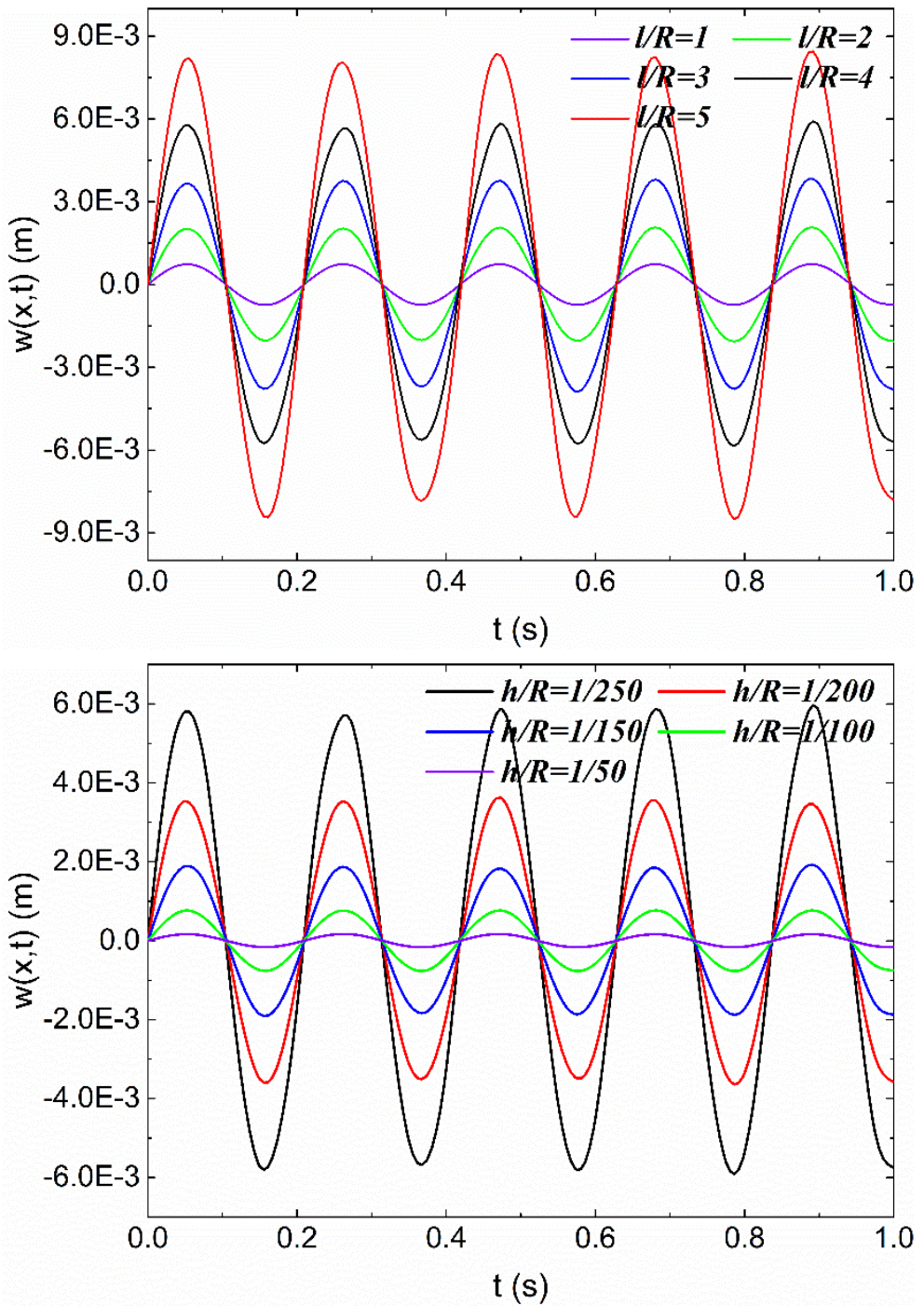

Fig. 7. The time-history responses of a cylindrical shell at a chosen position $(x=l / 2, \theta=0, z=0)$ under different length-radius ratios and thickness-radius ratios. 
figure1.tif
Click here to download high resolution image

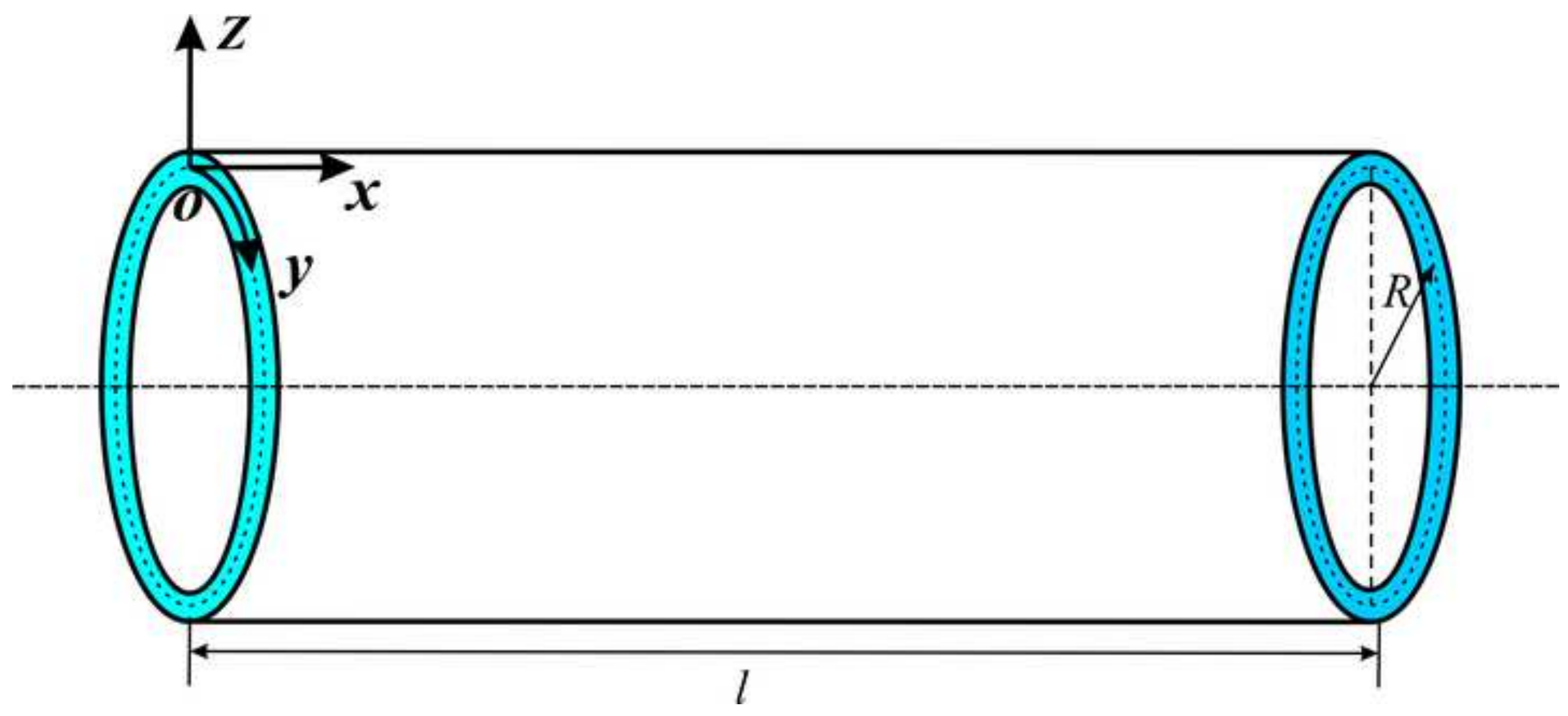


Click here to download high resolution image

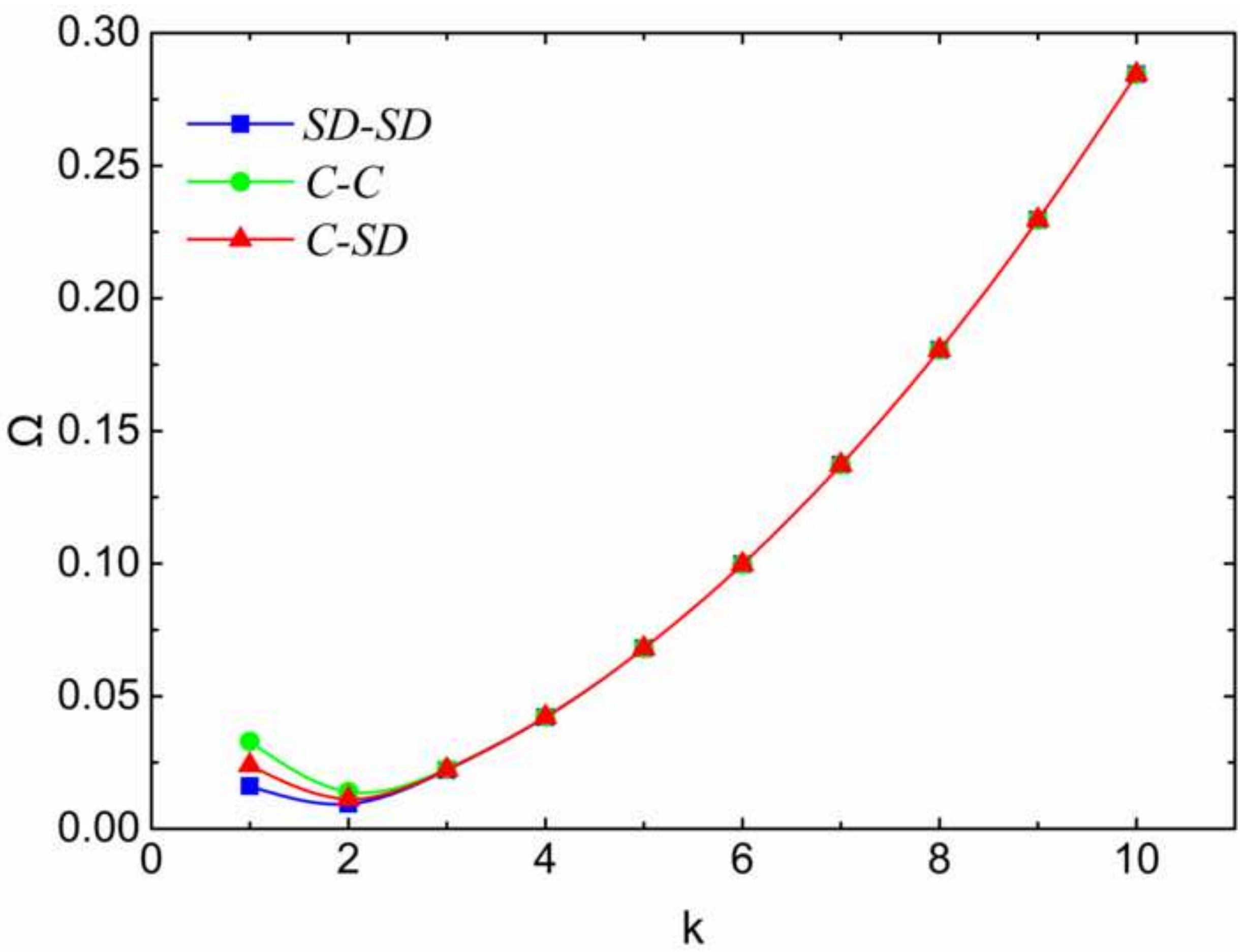


Click here to download high resolution image

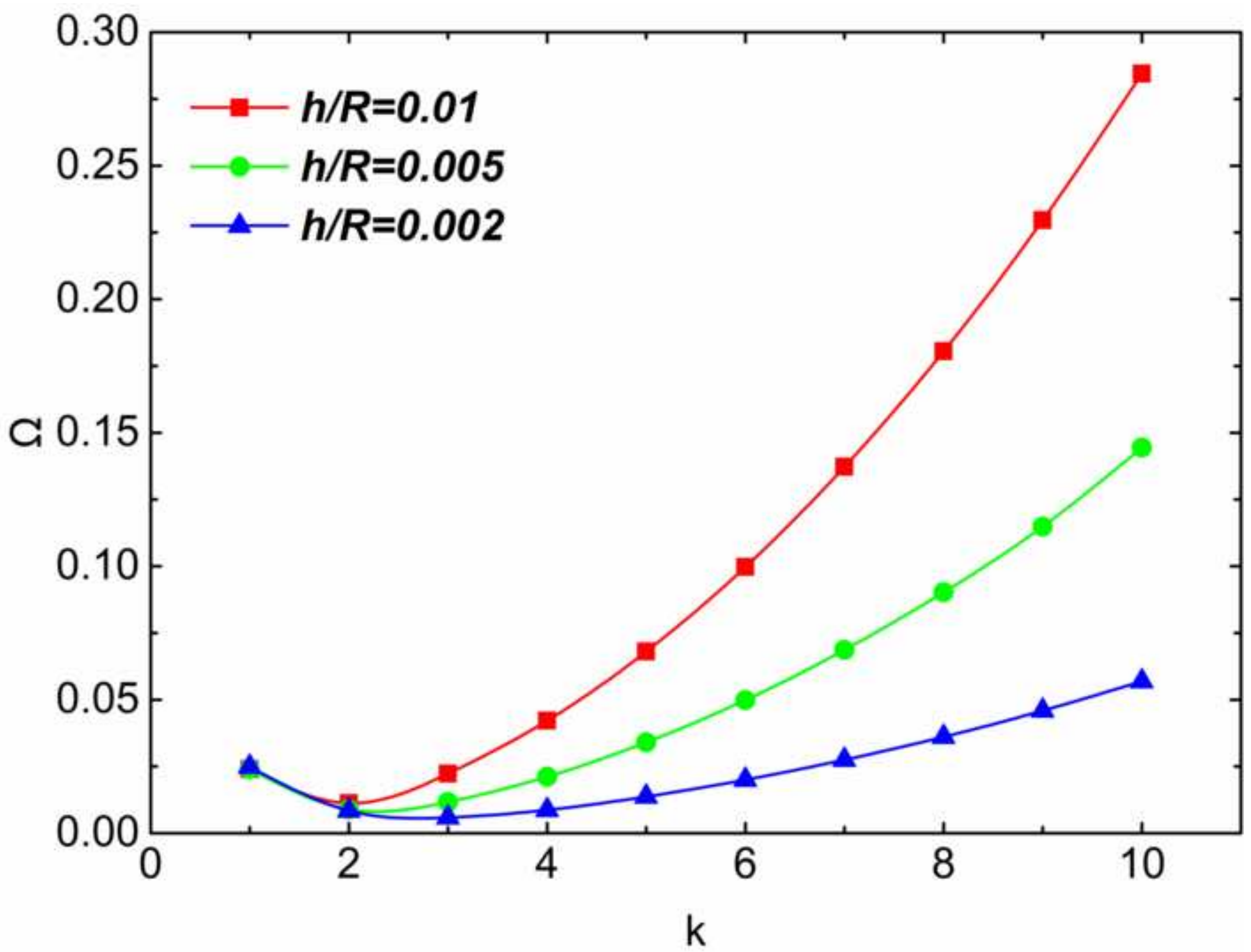


Click here to download high resolution image

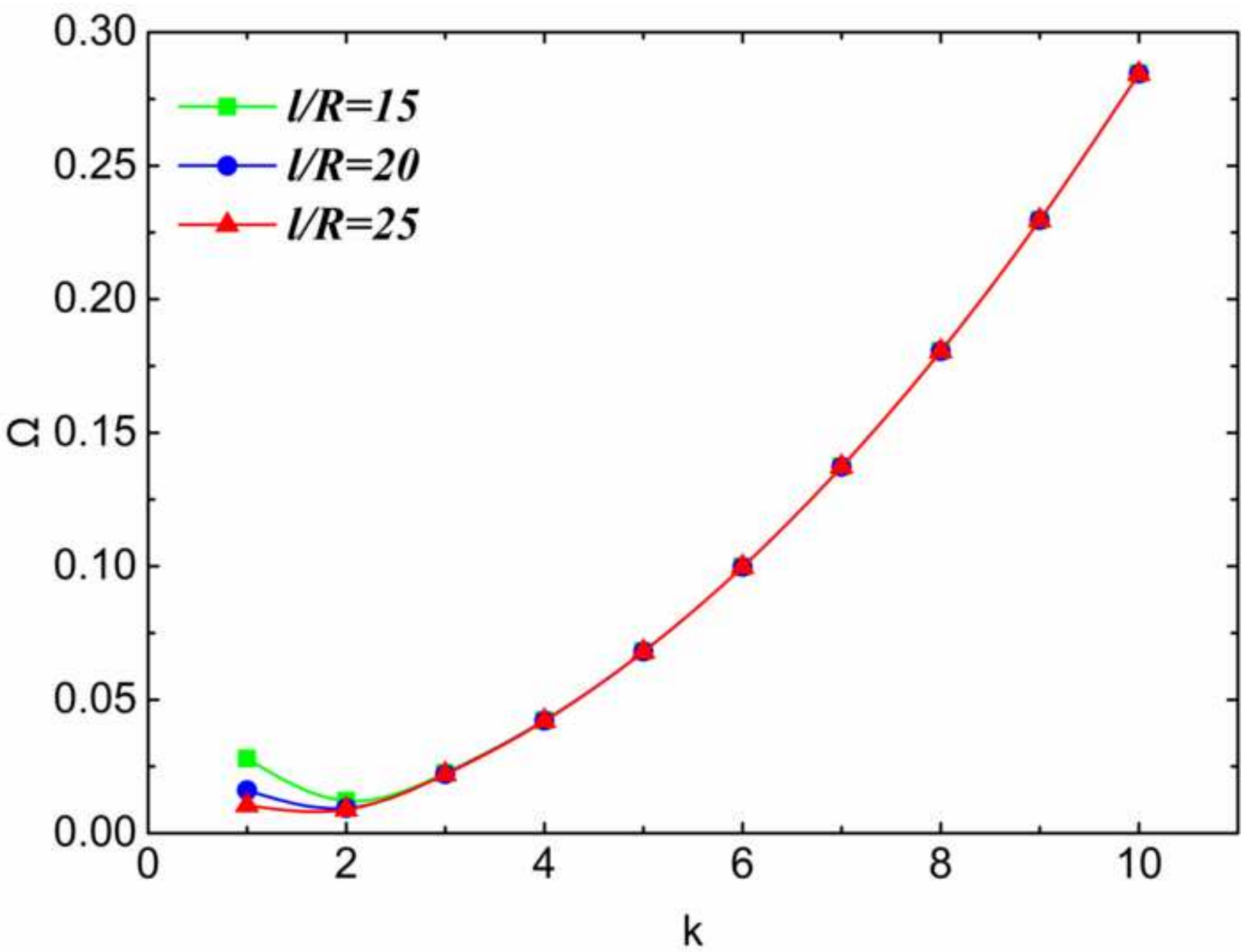




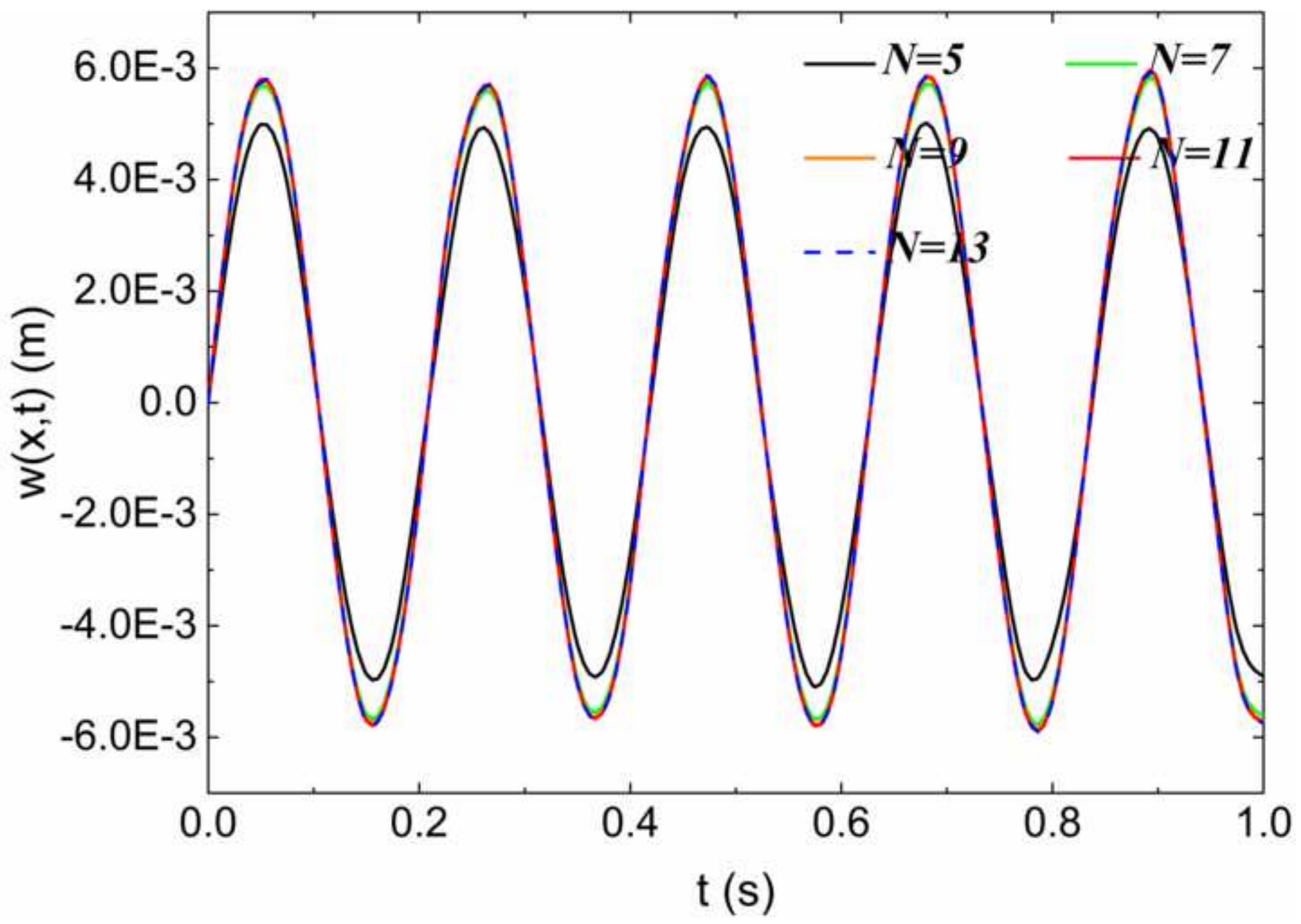




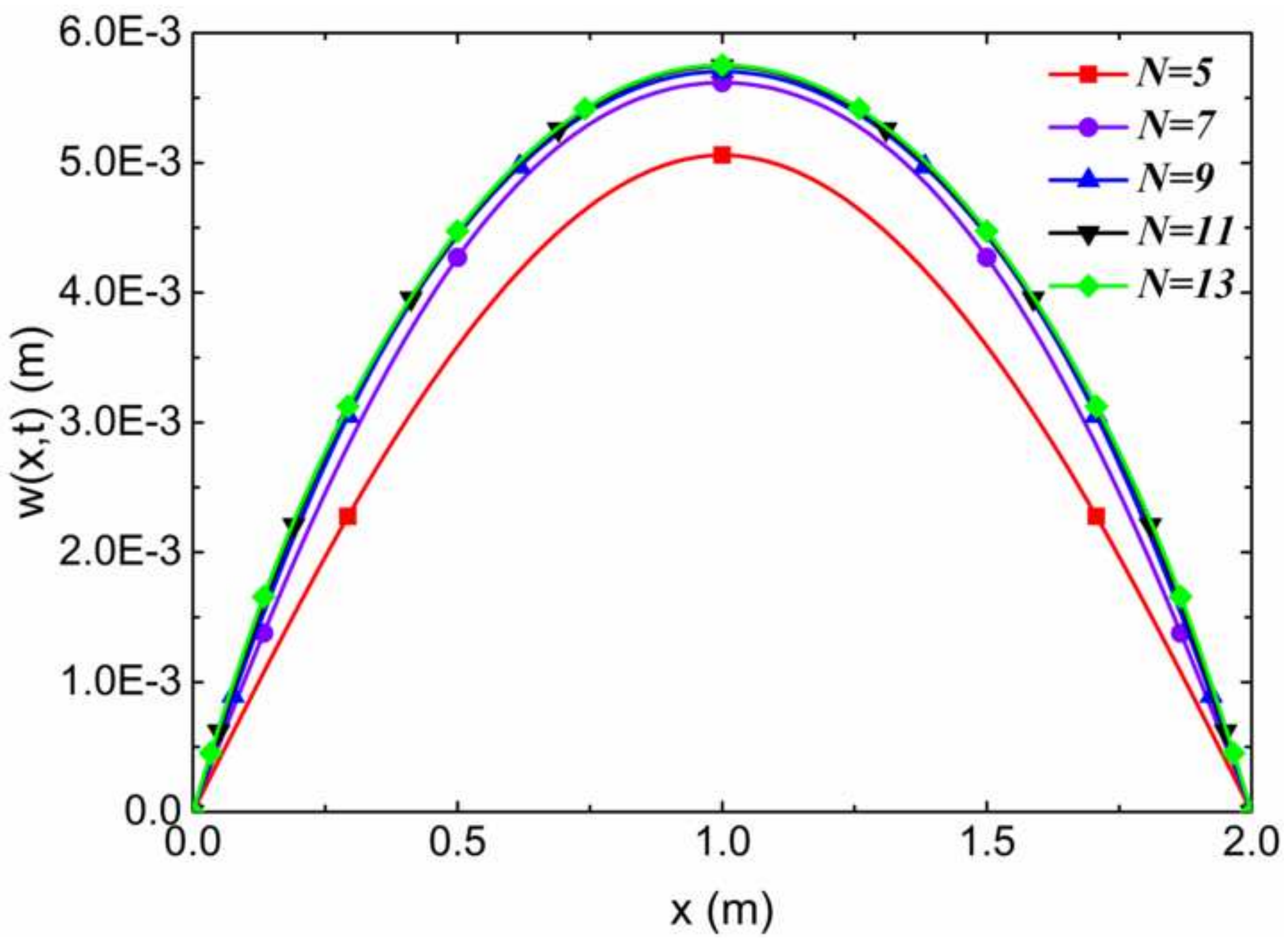




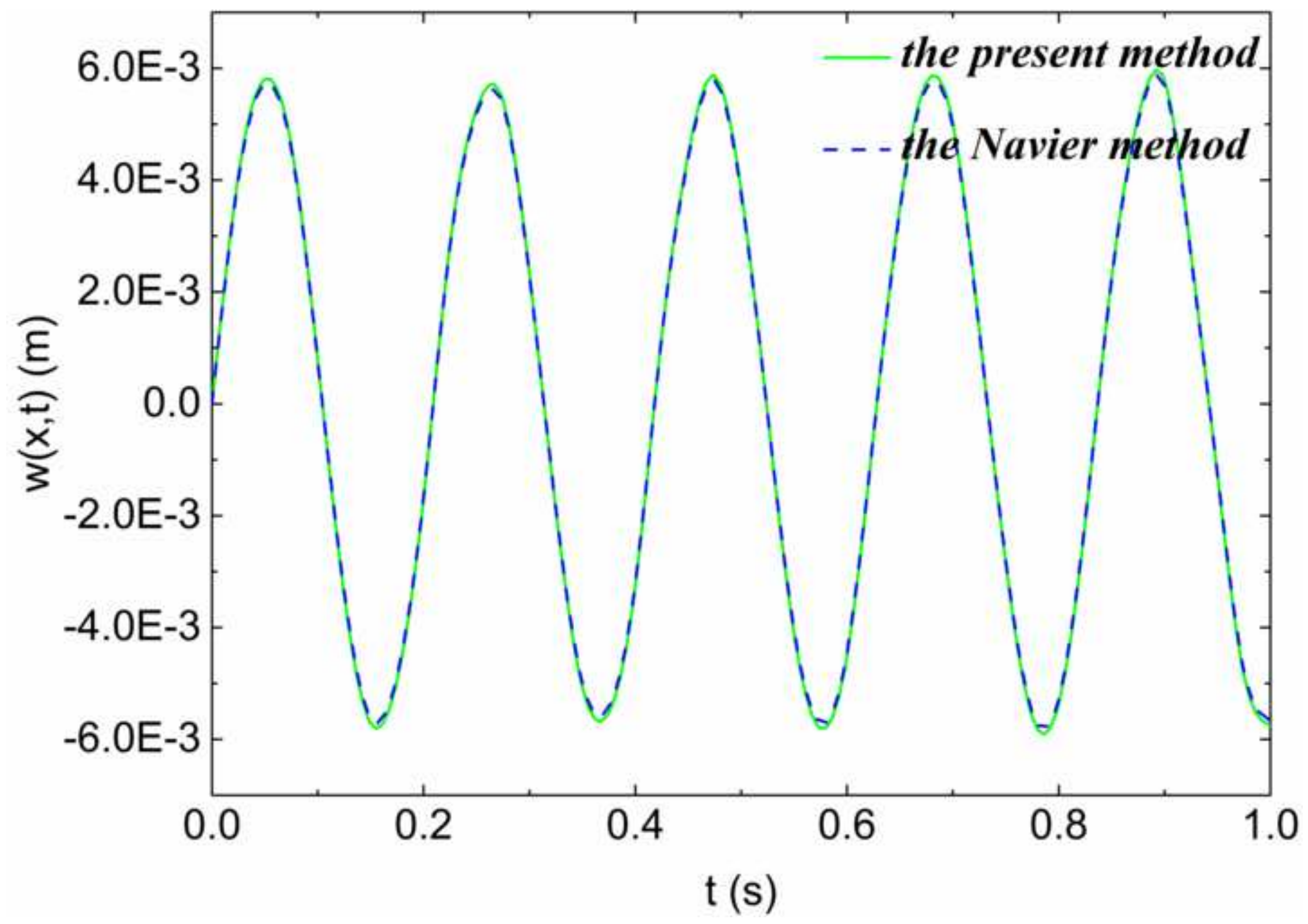




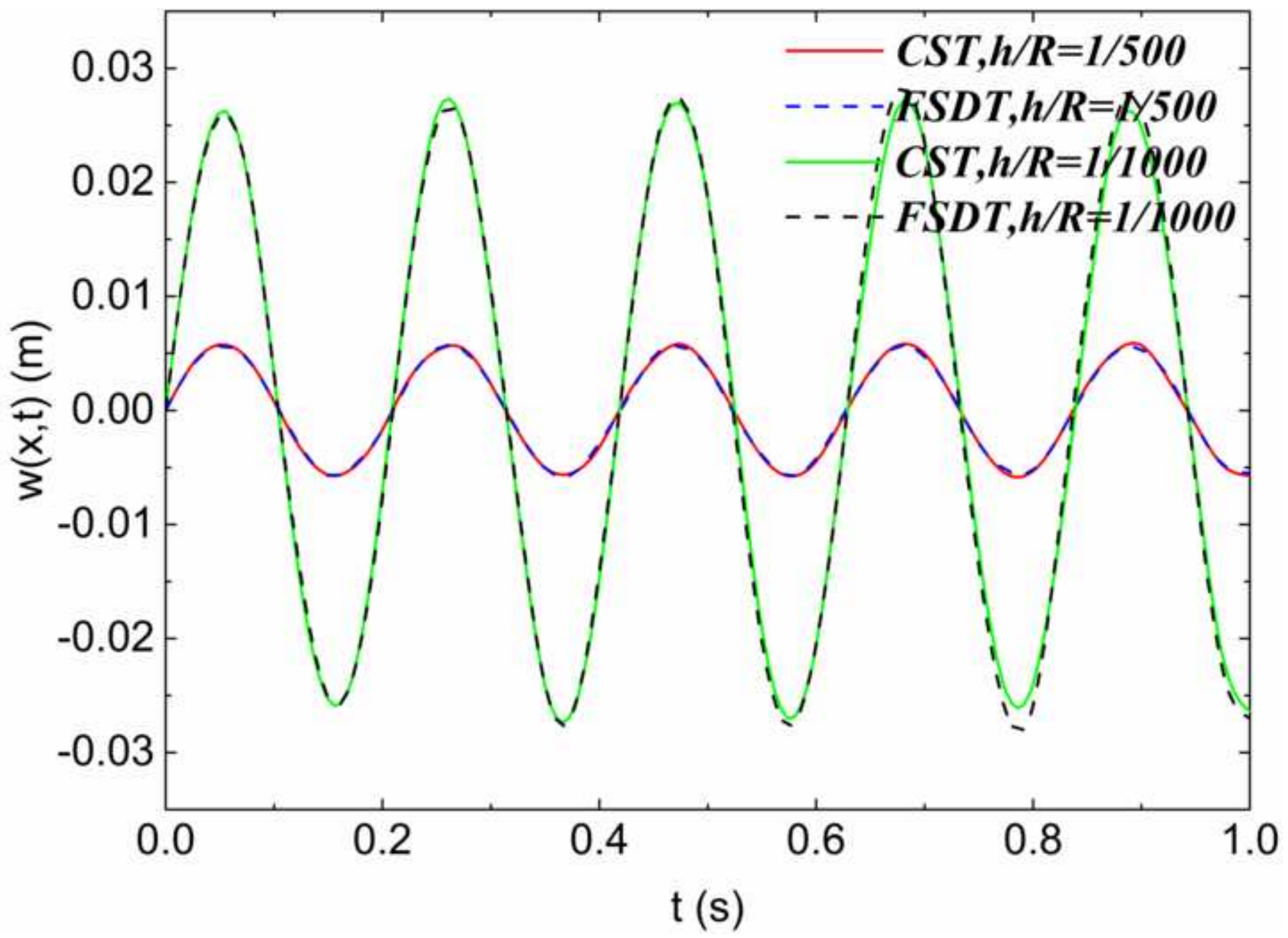




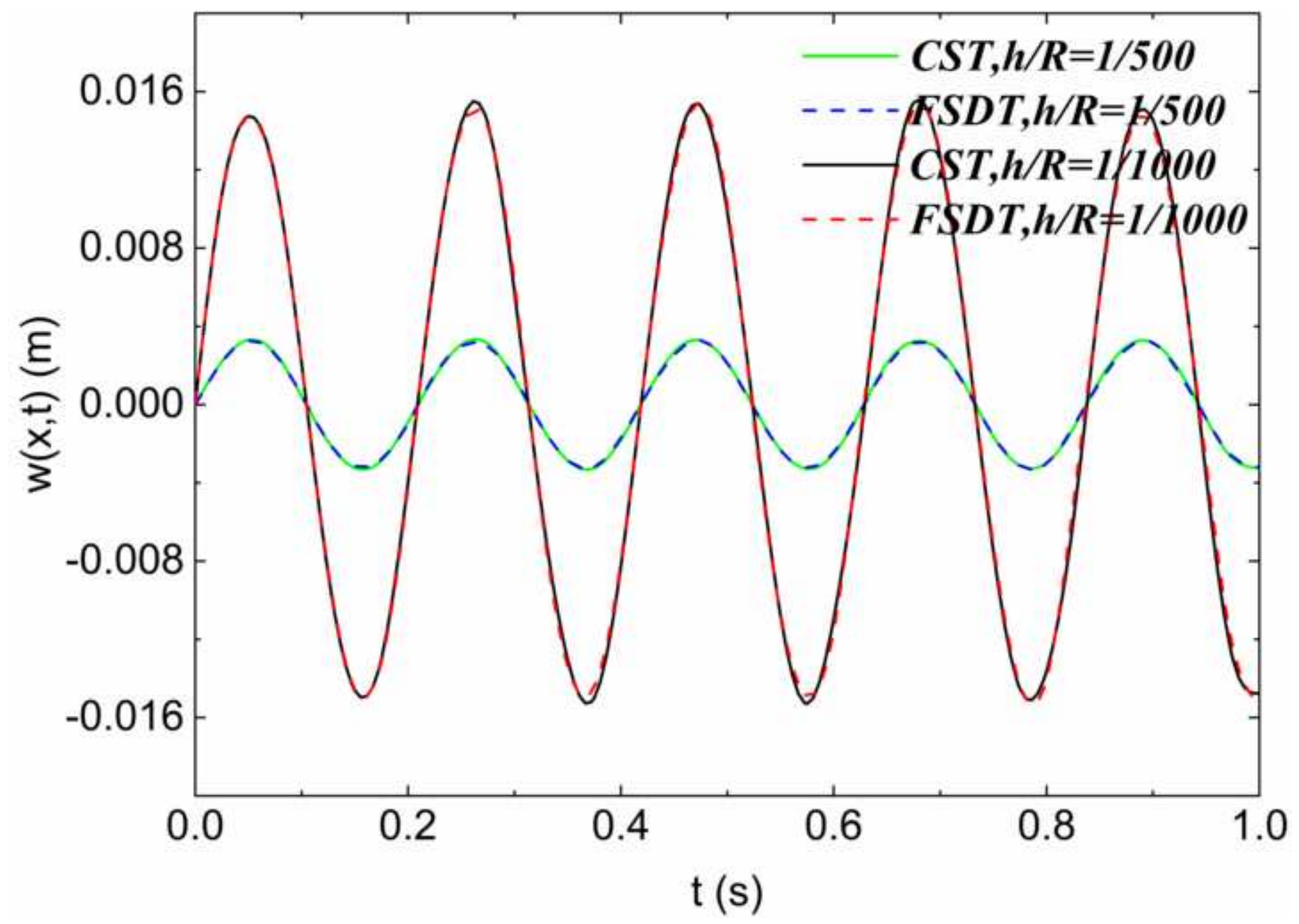




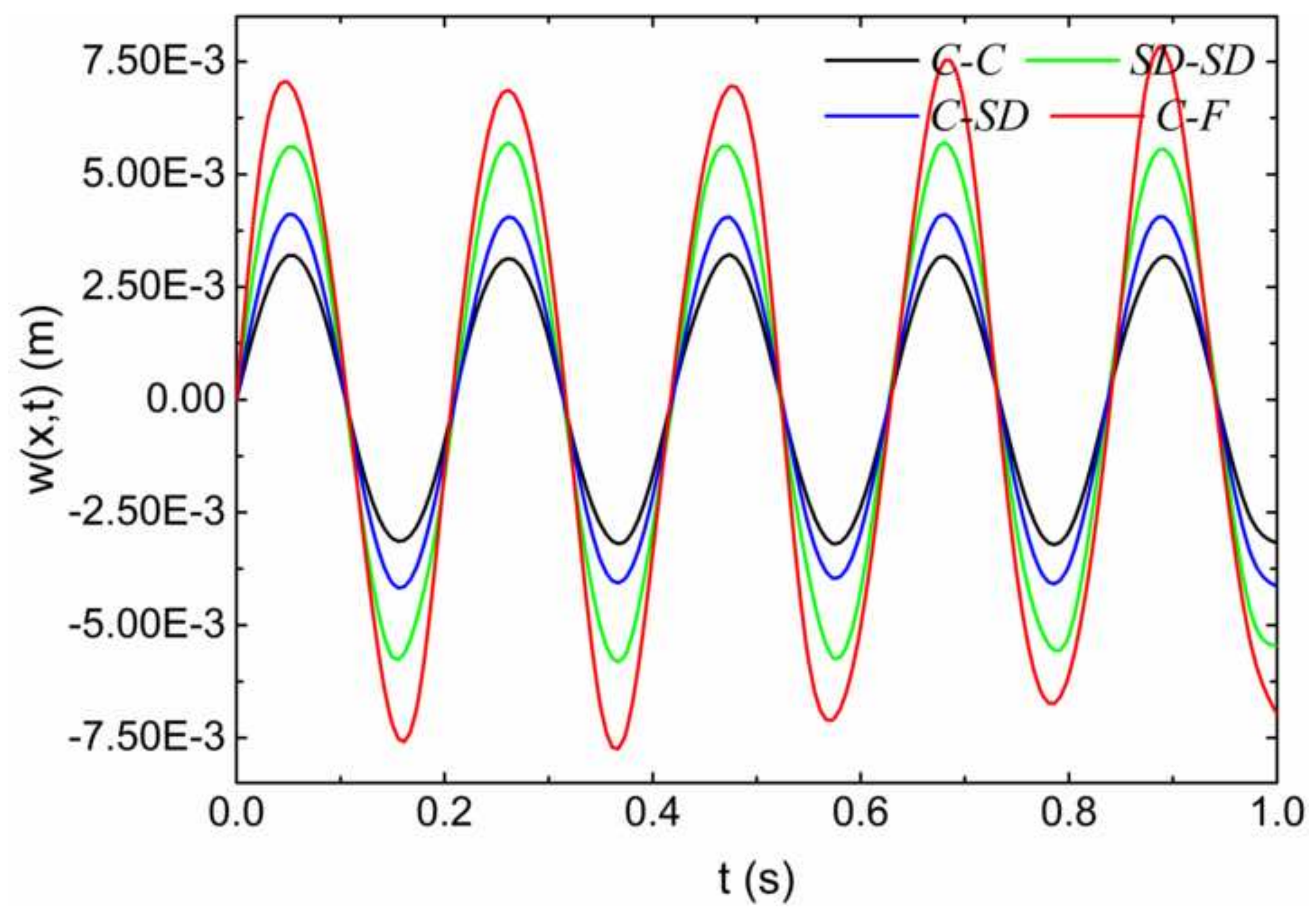




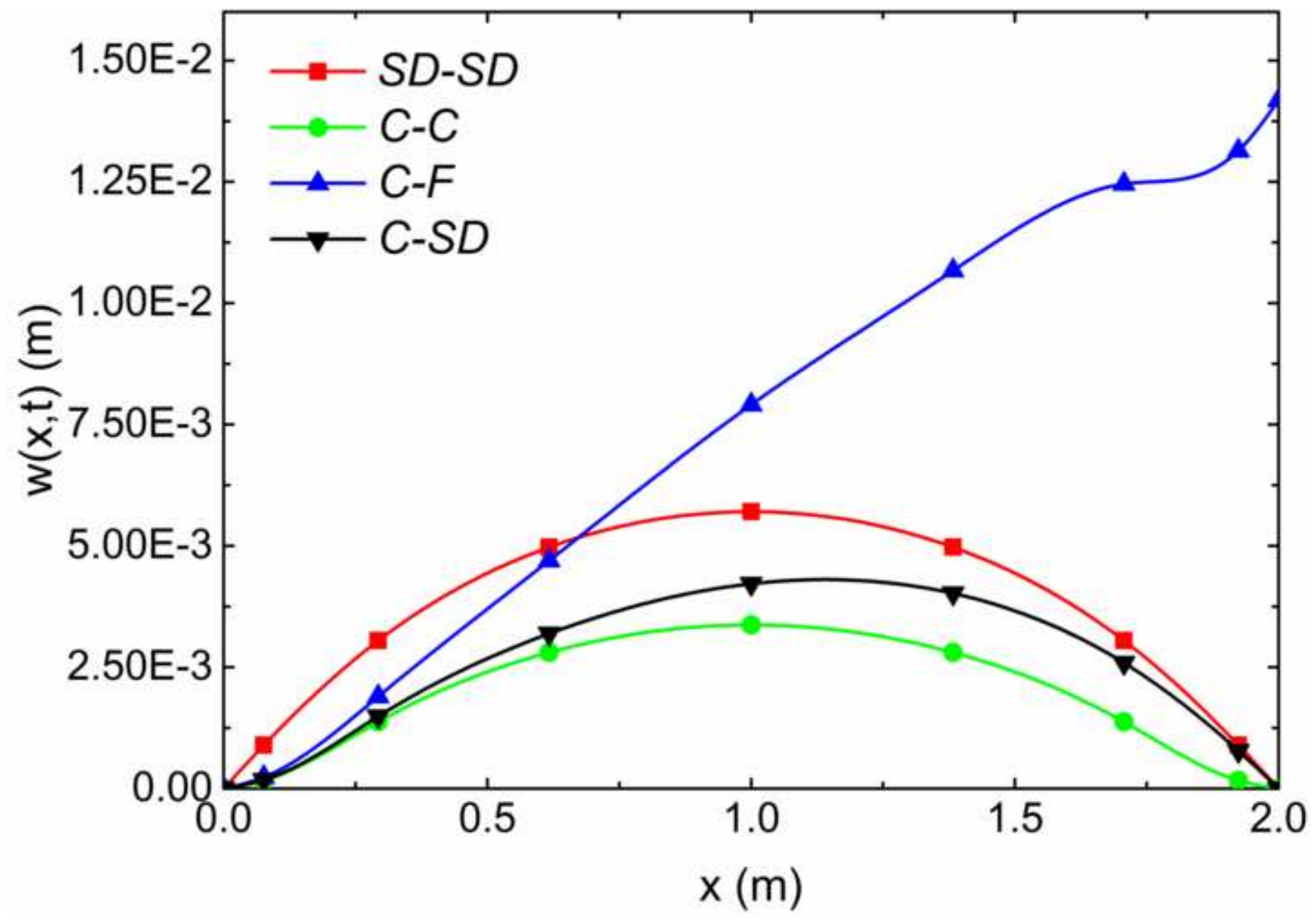




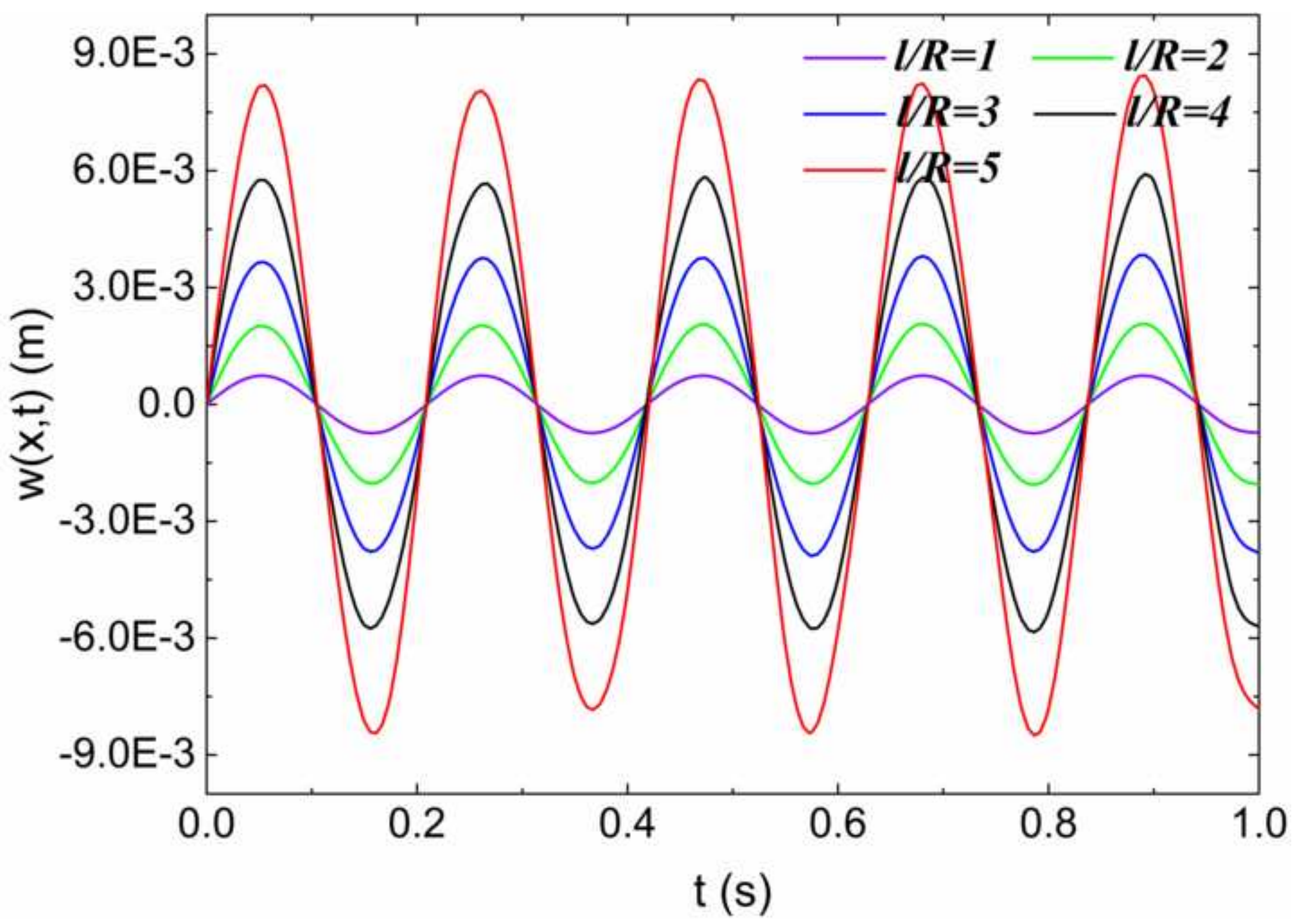




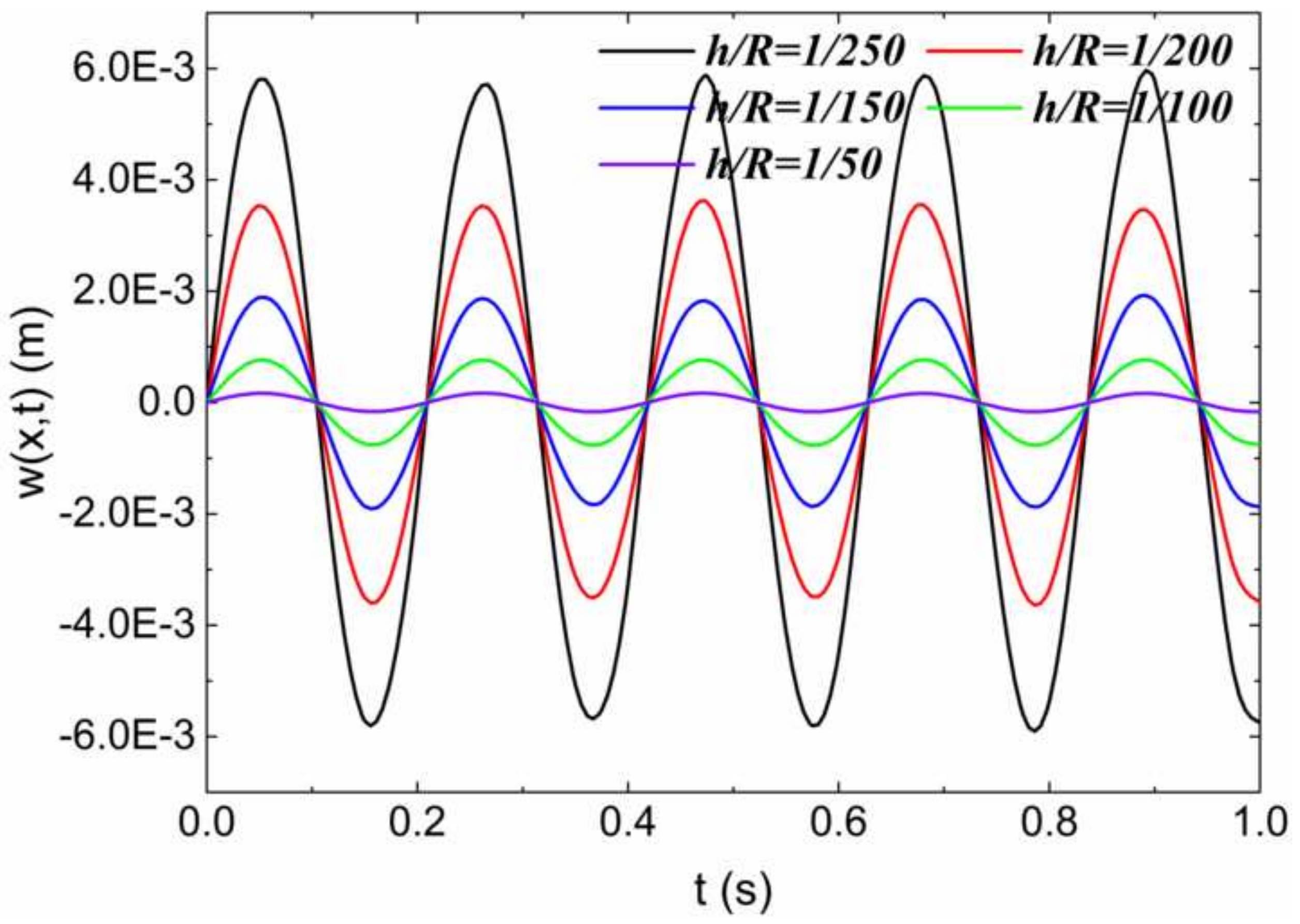

